\title{
Asymptotically Stable Running for a Five-Link, Four-Actuator, Planar Bipedal Robot
}

\author{
C. Chevallereau, E.R. Westervelt, and J.W. Grizzle
}

\begin{abstract}
Provably asymptotically stable running gaits are developed for the five-link, four-actuator bipedal robot, RABBIT. A controller is designed so that the Poincaré return map associated with periodic running gaits can be computed on the basis of a model with impulse-effects that, previously, had been used only for the design of walking gaits. This feedback design leads to the notion of a hybrid zero dynamics for running, which in turn allows the existence and stability of running gaits to be determined on the basis of a scalar map. The main results are illustrated via simulations performed on models with known parameters and on models with parameter uncertainty and structural changes. Animations of the resulting running motions are available on the web.
\end{abstract}

\section{INTRODUCTION}

$\mathbf{T}$ HIS paper addresses the design and analysis of asymptotically stable running gaits for RABBIT, a five-link, four-actuator, planar bipedal robot [1]. Running is defined as forward motion with alternation of single support (one leg on the ground) and flight (no contact with the ground); without forward motion, the robot would be jumping in place, or "jogging" in place. RABBIT was specifically designed to advance the fundamental understanding of controlled legged locomotion. In particular, it was designed without feet, and hence a widely-used stability heuristic, called the zero moment point (ZMP) criterion, is not applicable. The robot was designed to be able to walk with an average forward speed of at least $5 \mathrm{~km} / \mathrm{h}$ and to run at more than $12 \mathrm{~km} / \mathrm{h}$.

In a series of papers, the authors and colleagues have developed new feedback control strategies [2]-[7] that achieve provably asymptotically-stable walking gaits in underactuated bipeds, such as RABBIT, and demonstrated many of them experimentally [8], [9]. In regards to running, open-loop trajectories have been studied in [10], [11]. An objective of this paper is to develop a time-invariant feedback controller that realizes these open-loop running trajectories as provably asymptotically-stable orbits. A key step is to design the feedback controller in such a way that the Poincaré map associated with a running gait can be computed on the basis of the models with impulse effects studied in [3] for the design of walking gaits. This leads to the notion of a hybrid zero dynamics (HZD) for running, and to the closed-form computation of the restricted Poincaré return map [12].

C. Chevallereau is with the IRCCyN, Ecole Centrale de Nantes, UMR CNRS 6597, BP 92101, 1 rue de la Noë, 44321 Nantes, cedex 03, France, Email: Christine.Chevallereau @irccyn.ec-nantes.fr. E.R. Westervelt is with the Department of Mechanical Engineering, The Ohio State University, Columbus, Ohio 43210, USA, E-mail: westervelt.4@osu.edu. J.W. Grizzle is with the Control Systems Laboratory, EECS Department, University of Michigan, Ann Arbor, Michigan 48109-2122, USA, E-mail: grizzle@umich.edu.
Section III presents the models of the stance, flight, and impact phases of the studied robot. These are assembled into an overall hybrid model of running in Section IV. A qualitative discussion of the proposed control law for running is presented in Section V. The details of the control law are developed in Section VI. Like the model, the controller is hybrid, with both continuous-time and event-based actions taking place. In the stance phase, the control law is identical to the development in [3]. The control law in the flight phase consists of two parts: a continuous action similar to [3] plus a discrete or eventto-event action that adjusts the coefficients in the continuous portion of the controller in order to achieve a landing objective that assures the existence of a hybrid zero dynamics. The existence and stability properties of periodic orbits of the closed-loop hybrid model are studied in Section VII. The main contribution is the closed-form computation of the restricted Poincare return map of the hybrid zero dynamics, because analysis of orbits in the closed-loop, multi-phase, hybrid model can then be performed on the basis of an easily computable scalar map. Section VIII begins an extensive simulation study of the control laws proposed in the paper as applied to the underactuated, planar bipedal robot, RABBIT [1]. The restricted Poincare return map is computed for ten different running trajectories, varying from $0.5 \mathrm{~m} / \mathrm{s}$ to $2.75 \mathrm{~m} / \mathrm{s}$, showing the ability to achieve stable running motions over a wide range of speeds. The trajectories for $1.5 \mathrm{~m} / \mathrm{s}$ and $2.5 \mathrm{~m} / \mathrm{s}$ are illustrated in detail under the assumption of a perfect model. The robustness to model imperfection is studied via simulation in Section IX. The systematic design of auxiliary event-based control action [4], [13] to accelerate the rate of convergence to a periodic orbit is illustrated in Section $\mathrm{X}$. The paper is concluded in Section XI. Animations and other supporting material are available on the web [14].

\section{RELATED WORK}

In the early 1980's, Raibert proposed an elegant conceptualization of running in terms of a one-legged, prismatic-kneed hopper [15], [16]. He decomposed his control actions into three parts-hopping height, foot touchdown angle, and body posture-and emphasized the role of symmetry in designing stable running motions. The remarkable success of Raibert's control law motivated others to analytically characterize its stability [17], [18], and to further investigate the role of passive elements in achieving efficient running with a hopper [19]. Raibert's control scheme has been augmented with leg-coordination logic to achieve running in prismatic-kneed bipeds and quadrupeds [15], [20].

Various attempts have been made to extend a Raibert-style controller to a robot with revolute joints. Building on the 
ideas of Koditschek et al. [21]-[24], a spring-loaded invertedpendulum model-essentially a prismatic-kneed hopper with a radially symmetric body and a massless leg-was approximately embedded into a one-legged, revolute-jointed robot with an ankle, knee, and hip [25], [26]. With this approach, the center of mass of the robot could be nicely controlled. However, there was no natural "posture principle" to specify the evolution of the remaining degrees of freedom of the robot; also, the ballistic phase was difficult to address, due to underactuation and the non-holonomic constraint arising from conservation of angular momentum. Various methods for controlling the attitude of bodies undergoing ballistic motion have been studied; see [27]-[31] and references therein.

In late 2003, both Iguana Robotics and Sony announced (separate) experimental demonstrations of running for bipedal robots with revolute knees. In early 2004, running was announced for another humanoid robot, HRP-2LR [32], and in December 2004, Honda's robot, ASIMO, achieved running. The readers are invited to seek videos of these robots on the web. The controllers of the Sony and Honda robots are based on the ZMP, that of Iguana Robotics is based on central pattern generators (CPGs), and HRP-2LR uses "resolved momentum". To our best knowledge, only two other bipeds with revolute knees have been designed to perform running-Johnnie in Munich, Germany [33], [34] and RABBIT in Grenoble, France [1], [35].

The computation of optimal running trajectories has been studied in [36]. Trajectory tracking for running was investigated on a simulation model of the Honda biped, ASIMO, [37]. A nominal trajectory was computed off-line, and then during the ballistic phase, an on-line trajectory modification was made to allow Raibert-like control of foot placement. A control strategy for running that does not rely on trajectory tracking was studied in [38], where a Raibert-like controller was used on a planar robot with a torso and two prismatickneed legs. The control action sought to excite natural passive solutions of the dynamics by restoring energy lost at touchdown.

\section{Mechanical Model of A Biped Runner}

\section{A. The biped}

The studied bipedal robot evolves with respect to a flat surface in the sagittal plane; see Fig. 1. The flat surface will be referred to as the ground. The robot is composed of five rigid links with mass, connected through ideal (i.e., rigid and frictionless) revolute joints to form a torso and two identical legs, with each leg articulated by a knee. Each leg end is terminated in a point so that, in particular, the robot does not have feet. An actuator is provided at the each knee and two actuators are provided at the hips, acting between the torso and the legs. There is no actuation at the leg ends. Hence, in single support, the robot is underactuated.

The robot is said to be in flight phase when there is no contact with the ground, and in stance phase when one leg end is in stationary contact with the ground (that is, the leg end is acting as an ideal pivot) and the other leg is free. For the stance phase, the leg in contact with the ground is called the stance leg and the other leg is the swing leg.

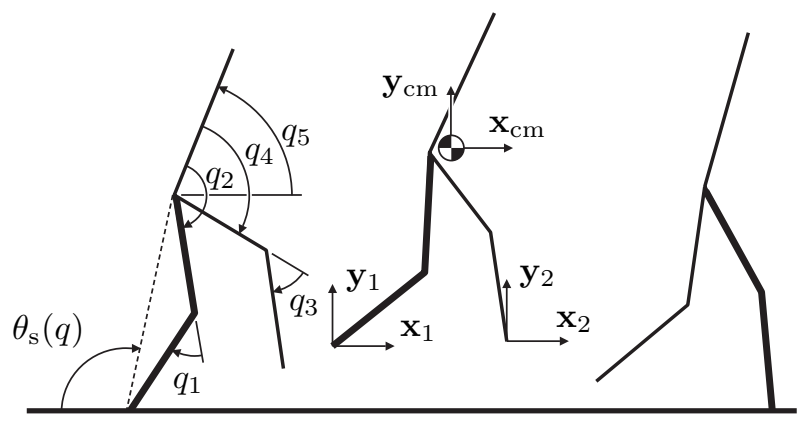

(a)

(b)

(c)

Fig. 1. Different phases of running with coordinate conventions labeled. The robot is shown (a) at the end of the stance phase; (b) during flight; and (c) at the beginning of the stance phase just after impact. To avoid clutter, the coordinate conventions have been spread out over the single support and flight phases even though they apply to all three phases. leg- 1 is presented in bold. Angles are positive in the clockwise direction.

In the flight phase, the robot has seven degrees of freedom (DOF): a degree of freedom associated with the orientation of each link, plus two DOF associated with the horizontal and vertical displacement of the center of mass within the sagittal plane. The state vector of the dynamical model is thus 14dimensional: there are seven configuration variables required to describe the position of the robot, plus the associated velocities. In the stance phase, the robot has only five DOF because the position of the center of mass is determined by the orientation of the five links (plus a horizontal, constant off-set of the stance leg end with respect to a world frame). The state vector of the dynamical model is thus 10 -dimensional.

\section{B. Lagrangian model for flight}

A convenient choice of configuration variables is depicted in Fig. 1. The vector of body coordinates $q_{\mathrm{b}}$ consisting of the relative angles $\left(q_{1}, q_{2}, q_{3}, q_{4}\right)^{\prime}$ describes the shape of the biped. The biped's absolute orientation with respect to the world frame is given by $q_{5}$. The biped's absolute position is specified by the Cartesian coordinates of the center of mass, $\left(\mathbf{x}_{\mathrm{cm}}, \mathbf{y}_{\mathrm{cm}}\right)$. The vector of generalized coordinates is denoted as $q_{\mathrm{f}}:=\left(q_{\mathrm{b}}^{\prime}, q_{5}, \mathbf{x}_{\mathrm{cm}}, \mathbf{y}_{\mathrm{cm}}\right)^{\prime}$.

The dynamic model is easily obtained with the method of Lagrange, which consists of first computing the kinetic energy and potential energy of each link, and then summing terms to compute the total kinetic energy, $K_{\mathrm{f}}$, and the total potential energy, $V_{\mathrm{f}}$ [39]-[41]. The Lagrangian is defined as $\mathcal{L}_{\mathrm{f}}=K_{\mathrm{f}}-$ $V_{\mathrm{f}}$, and the dynamical model is determined from Lagrange's equation

$$
\frac{d}{d t} \frac{\partial \mathcal{L}_{\mathrm{f}}}{\partial \dot{q}_{\mathrm{f}}}-\frac{\partial \mathcal{L}_{\mathrm{f}}}{\partial q_{\mathrm{f}}}=\Gamma_{\mathrm{f}}
$$

where $\Gamma_{f}$ is the vector of generalized forces and torques applied to the robot.

In terms of the generalized coordinates of the robot, $q_{\mathrm{f}}$, the total kinetic energy becomes

$$
K_{\mathrm{f}}=\frac{1}{2} \dot{q}_{\mathrm{f}}^{\prime} D_{\mathrm{f}}\left(q_{\mathrm{b}}\right) \dot{q}_{\mathrm{f}}
$$


where

$$
D_{\mathrm{f}}=\left[\begin{array}{cc}
A\left(q_{\mathrm{b}}\right) & 0_{5 \times 2} \\
0_{2 \times 5} & m I_{2 \times 2}
\end{array}\right],
$$

$m$ is the total mass of the robot, and $A$ depends only on $q_{\mathrm{b}}$ because the total kinetic energy is invariant under rotations and translations of the body. The potential energy is

$$
V_{\mathrm{f}}=m g \mathbf{y}_{\mathrm{cm}} .
$$

The principle of virtual work yields that the external torques are

$$
\Gamma_{\mathrm{f}}=B_{\mathrm{f}} u=\left[\begin{array}{c}
I_{4 \times 4} \\
0_{3 \times 4}
\end{array}\right] u
$$

where $u$ is the vector of actuator torques applied at the four joints of the robot. Applying Lagrange's equation leads to a model of the form

$$
D_{\mathrm{f}}\left(q_{\mathrm{b}}\right) \ddot{q}_{\mathrm{f}}+C_{\mathrm{f}}\left(q_{\mathrm{b}}, \dot{q}_{\mathrm{f}}\right) \dot{q}_{\mathrm{f}}+G_{\mathrm{f}}\left(q_{\mathrm{f}}\right)=B_{\mathrm{f}} u,
$$

where $D_{\mathrm{f}}$ is the inertia matrix, the matrix $C_{\mathrm{f}}$ contains Coriolis and centrifugal terms, and $G_{\mathrm{f}}$ is the gravity vector. Introducing the state vector $x_{\mathrm{f}}:=\left(q_{\mathrm{f}}^{\prime}, \dot{q}_{\mathrm{f}}^{\prime}\right)^{\prime}$, the Lagrangian model (6) is easily expressed as

$$
\dot{x}_{\mathrm{f}}=f_{\mathrm{f}}\left(x_{\mathrm{f}}\right)+g_{\mathrm{f}}\left(x_{\mathrm{f}}\right) u .
$$

The state space is taken as $T \mathcal{Q}_{\mathrm{f}}:=\left\{x_{\mathrm{f}}:=\left(q_{\mathrm{f}}^{\prime}, \dot{q}_{\mathrm{f}}^{\prime}\right)^{\prime} \mid q_{\mathrm{f}} \in\right.$ $\left.\mathcal{Q}_{\mathrm{f}}, \dot{q}_{\mathrm{f}} \in \mathbb{R}^{7}\right\}$, where, if $\mathbb{S}$ denotes the circle, the configuration space $\mathcal{Q}_{\mathrm{f}}$ is a simply-connected, open subset of $\mathbb{S}^{5} \times \mathbb{R}^{2}$ corresponding to physically reasonable configurations of the robot.

\section{Lagrangian model for stance}

For the stance phase, the generalized coordinates can be taken as $q:=\left(q_{\mathrm{b}}^{\prime}, q_{5}\right)^{\prime}=\left(q_{1}, \cdots, q_{5}\right)^{\prime}$. Since the robot's legs are identical, in the stance phase, it will be assumed without loss of generality that leg-1 is in contact with the ground. Moreover, the Cartesian position of the stance leg end will be identified with the origin of the $(x-y)$-axes of a world frame.

The position of the center of mass can be expressed in terms of $q$ per

$$
\left[\begin{array}{l}
\mathbf{x}_{\mathrm{cm}}(q) \\
\mathbf{y}_{\mathrm{cm}}(q)
\end{array}\right]=\mathbf{f}_{1}(q)
$$

where $\mathbf{f}_{1}$ is determined from the robot's geometric parameters (link lengths, masses, positions of the centers of mass). Hence

$$
\dot{q}_{\mathrm{f}}=\left[\begin{array}{c}
I_{5 \times 5} \\
\frac{\partial \mathbf{f}_{1}}{\partial q}
\end{array}\right] \dot{q} .
$$

Substituting (9) into (2) yields the kinetic energy of the stance phase,

$$
K_{\mathrm{s}}=\frac{1}{2} \dot{q}^{\prime} D_{\mathrm{s}}\left(q_{\mathrm{b}}\right) \dot{q}
$$

with

$$
D_{\mathrm{s}}\left(q_{\mathrm{b}}\right)=A\left(q_{\mathrm{b}}\right)+m \frac{\partial \mathbf{f}_{1}(q)^{\prime}}{\partial q} \frac{\partial \mathbf{f}_{1}(q)}{\partial q} ;
$$

because the kinetic energy is invariant under rotations of the body, $D_{\mathrm{s}}$ depends only on $q_{\mathrm{b}}$. The potential energy remains $V_{\mathrm{s}}(q)=m g \mathbf{y}_{\mathrm{cm}}(q)$. Lagrange's equation becomes

$$
\frac{d}{d t} \frac{\partial \mathcal{L}_{\mathrm{s}}}{\partial \dot{q}}-\frac{\partial \mathcal{L}_{\mathrm{s}}}{\partial q}=\Gamma_{\mathrm{s}},
$$

and the external torques are

$$
\Gamma_{\mathrm{s}}=B_{\mathrm{s}} u=\left[\begin{array}{c}
I_{4 \times 4} \\
0_{1 \times 4}
\end{array}\right] u .
$$

The dynamic model can therefore be written as

$$
D_{\mathrm{s}}\left(q_{\mathrm{b}}\right) \ddot{q}+C_{\mathrm{s}}\left(q_{\mathrm{b}}, \dot{q}\right) \dot{q}+G_{\mathrm{s}}(q)=B_{\mathrm{s}} u .
$$

Introducing the state vector $x_{\mathrm{s}}:=\left(q^{\prime}, \dot{q}^{\prime}\right)^{\prime}$, the Lagrangian model (14) is easily expressed as

$$
\dot{x}_{\mathrm{s}}=f_{\mathrm{s}}\left(x_{\mathrm{s}}\right)+g_{\mathrm{s}}\left(x_{\mathrm{s}}\right) u \text {. }
$$

The state space is taken as $T \mathcal{Q}_{\mathrm{s}}:=\left\{x_{\mathrm{s}}:=\left(q^{\prime}, \dot{q}^{\prime}\right)^{\prime} \mid q \in\right.$ $\left.\mathcal{Q}_{\mathrm{s}}, \dot{q} \in \mathbb{R}^{5}\right\}$, where the configuration space $\mathcal{Q}_{\mathrm{s}}$ is a simplyconnected, open subset of $\mathbb{S}^{5}$ corresponding to physically reasonable configurations.

\section{The impact model}

The Cartesian position of the end of leg-2 can be expressed in terms of the Cartesian position of the center of mass and the robot's angular coordinates as

$$
\left[\begin{array}{l}
\mathbf{x}_{2} \\
\mathbf{y}_{2}
\end{array}\right]=\left[\begin{array}{l}
\mathbf{x}_{\mathrm{cm}} \\
\mathbf{y}_{\mathrm{cm}}
\end{array}\right]-\mathbf{f}_{2}(q),
$$

where $\mathbf{f}_{2}$ is determined from the robot's parameters (links lengths, masses, positions of the centers of mass); see (8). When leg-2 touches the ground at the end of a flight phase, an impact takes place. The impact model of [42], [43] is used, which represents the ground reaction forces at impact as impulses with intensity $I_{R}$. The impact is assumed inelastic, with the velocity of the contact leg end becoming zero instantaneously; furthermore, after impact, the contact leg end is assumed to act as an ideal pivot. This model yields that the robot's configuration $q_{\mathrm{f}}$ is unchanged during impact and there are instantaneous changes in the velocities.

The velocity vector just before impact is denoted by $\dot{q}_{\mathrm{f}}^{-}$. After impact, with the assumption that the leg neither rebounds nor slides after impact, the robot is in stance phase. leg-2 acts as an ideal pivot and thus the linear velocity of the center of mass center can be expressed in terms of the angular velocities just after impact, $\dot{q}^{+}$, yielding

$$
\left[\begin{array}{l}
0 \\
0
\end{array}\right]=\left[\begin{array}{c}
\dot{\mathbf{x}}_{\mathrm{cm}}^{+} \\
\dot{\mathbf{y}}_{\mathrm{cm}}^{+}
\end{array}\right]-\frac{\partial \mathbf{f}_{2}(q)}{\partial q} \dot{q}^{+} .
$$

The impact model of [42], [43] is expressed as

$$
\left[\begin{array}{cc}
A\left(q_{\mathrm{b}}\right) & 0_{5 \times 2} \\
0_{2 \times 5} & m I_{2 \times 2}
\end{array}\right]\left(\left[\begin{array}{c}
\dot{q}^{+} \\
\dot{\mathbf{x}}_{\mathrm{cm}}^{+} \\
\dot{\mathbf{y}}_{\mathrm{cm}}^{+}
\end{array}\right]-\dot{q}_{\mathrm{f}}^{-}\right)=\left[\begin{array}{c}
\frac{-\partial \mathbf{f}_{2}^{\prime}(q)}{\partial q} \\
I_{2 \times 2}
\end{array}\right] I_{R} .
$$


The vector $I_{R}$ of the ground reaction impulses can be expressed using the last two lines of the matrix equation (18) in combination with (17):

$$
I_{R}=m\left(\frac{\partial \mathbf{f}_{2}(q)}{\partial q} \dot{q}^{+}-\left[\begin{array}{c}
\dot{\mathbf{x}}_{\mathrm{cm}}^{-} \\
\dot{\mathbf{y}}_{\mathrm{cm}}^{-}
\end{array}\right]\right) \text {. }
$$

Substituting this into the first five lines of (18) and rearranging yields that the robot's angular velocity vector after impact is given by a linear expression with respect to the velocity before impact:

$$
\dot{q}^{+}=\left[A+m \frac{\partial \mathbf{f}_{2}{ }^{\prime}}{\partial q} \frac{\partial \mathbf{f}_{2}}{\partial q}\right]^{-1}\left[A \mid m \frac{\partial \mathbf{f}_{2}{ }^{\prime}}{\partial q}\right] \dot{q}_{\mathrm{f}}^{-},
$$

which, for later use, is written as

$$
\dot{q}^{+}=\tilde{\Delta}\left(q_{\mathrm{f}}^{-}, \dot{q}_{\mathrm{f}}^{-}\right) .
$$

Remark 1: In the case of running, since the robot has 7 DOF before impact and only 5 DOF after impact, for any postimpact velocity, there is a two-dimensional set of velocities in the flight phase that gets mapped onto that same vector. This is different from walking where, generically, the double support impact results in a one-to-one mapping between pre-impact and post-impact velocity vectors.

\section{E. Some linear and angular momentum relationships}

A few linear and angular momentum properties of the mechanical models are noted. Let $\sigma_{\mathrm{cm}}$ denote the angular momentum of the biped about its center of mass. In the flight phase, $\sigma_{\mathrm{cm}}$ can be computed by $\sigma_{\mathrm{cm}}=\frac{\partial K_{\mathrm{f}}}{\partial \dot{q}_{5}}=A_{5} \dot{q}$, where $A_{5}$ is the fifth row of $A$. The fifth row of (1) yields conservation of $\sigma_{\mathrm{cm}}$,

$$
\dot{\sigma}_{\mathrm{cm}}=0 \text {. }
$$

In addition, the last two rows of (1) correspond to Newton's second law in a central gravity field:

$$
m \ddot{\mathbf{x}}_{\mathrm{cm}}=0 \quad \text { and } \quad m \ddot{\mathbf{y}}_{\mathrm{cm}}=-m g .
$$

Let $\sigma_{i}$ denote the angular momentum of the biped about the end of leg-i, for $i=1,2$. The three angular momenta are related by

$$
\sigma_{i}=\sigma_{\mathrm{cm}}+m\left(\left(\mathbf{y}_{\mathrm{cm}}-\mathbf{y}_{i}\right) \dot{\mathbf{x}}_{\mathrm{cm}}-\left(\mathbf{x}_{\mathrm{cm}}-\mathbf{x}_{i}\right) \dot{\mathbf{y}}_{\mathrm{cm}}\right) .
$$

This expression is valid in both the stance and flight phases. In the stance phase, $\sigma_{1}$ is determined by $\sigma_{1}=\frac{\partial K_{\mathrm{s}}}{\partial \dot{q}_{5}}=D_{\mathrm{s}, 5} \dot{q}$, where $D_{\mathrm{s}, 5}$ is the fifth row of $D_{\mathrm{s}}$. The fifth row of (12) yields the angular momentum balance theorem:

$$
\dot{\sigma}_{1}=-\frac{\partial V_{\mathrm{s}}}{\partial q_{5}}=m g \mathbf{x}_{\mathrm{cm}} .
$$

The impact model of [42], [43] yields conservation of angular momentum about the impact point. Indeed, because the only external impulsive force is applied at the impact point, the fifth row of (18) can be written as

$$
\sigma_{\mathrm{cm}}^{+}-\sigma_{\mathrm{cm}}^{-}=m\left[\mathbf{y}_{\mathrm{cm}}-\mathbf{y}_{2} \mid-\mathbf{x}_{\mathrm{cm}}+\mathbf{x}_{2}\right]\left[\begin{array}{c}
\dot{\mathbf{x}}_{\mathrm{cm}}^{+}-\dot{\mathbf{x}}_{\mathrm{cm}}^{-} \\
\dot{\mathbf{y}}_{\mathrm{cm}}^{+}-\dot{\mathbf{y}}_{\mathrm{cm}}^{-}
\end{array}\right],
$$

since $\frac{\partial f_{2}}{\partial q_{5}}=\left[-\mathbf{x}_{\mathrm{cm}}(q) \mid \mathbf{y}_{\mathrm{cm}}(q)\right]^{\prime}$. Using (24) results in

$$
\sigma_{2}^{+}=\sigma_{2}^{-},
$$

meaning the value of $\sigma_{2}$ is unchanged during the impact. Since the stance phase model assumes that the stance leg is leg-1, for later use, (27) is rewritten as

$$
\sigma_{1}^{\mathrm{s}+}=\sigma_{2}^{\mathrm{f}-}
$$

to reflect the swapping of the roles of the legs; see (30).

Remark 2: The notation $\mathrm{s}+$ emphasizes that $\sigma_{1}$ is being evaluated at the beginning of the stance phase and the notation $\mathrm{f}-$ emphasizes that $\sigma_{2}$ is being evaluated at the end of the flight phase. If no confusion is possible, the notation + and will be used. For example, the variable $\theta_{\mathrm{s}}$ only makes sense in the stance phase, and hence $\theta_{\mathrm{s}}^{\mathrm{s}-}$ would be redundant. On the other hand, for a variable such as $\mathbf{x}_{\mathrm{cm}}$, it is important to distinguish among $\mathbf{x}_{\mathrm{cm}}^{\mathrm{s}+}, \mathbf{x}_{\mathrm{cm}}^{\mathrm{s}-}, \mathbf{x}_{\mathrm{cm}}^{\mathrm{f}+}$, and $\mathbf{x}_{\mathrm{cm}}^{\mathrm{f}-}$.

Remark 3: The robot is assumed to advance from left to right that is, in the positive direction of the $\mathrm{x}$-coordinate. In this paper, angles are positive when measured in the clockwise direction so that the angular momenta about the stance leg end and the center of mass will be positive in the simulations. A more classical choice of measuring the angles in the trigonometric sense, that is, positive is counter clockwise, would lead to negative angular momenta for left-toright movement of the robot. In this case, (24) would become $\sigma_{i}=\sigma_{\mathrm{cm}}+m\left(\left(\mathbf{x}_{\mathrm{cm}}-\mathbf{x}_{i}\right) \dot{\mathbf{y}}_{\mathrm{cm}}-\left(\mathbf{y}_{\mathrm{cm}}-\mathbf{y}_{i}\right) \dot{\mathbf{x}}_{\mathrm{cm}}\right)$. In turn, certain equations derived from this one would have to be modified. For the convenience of the reader, the principal changes are noted in Appendix VI.

\section{HyBRID MOdEl OF RUNNING}

The overall biped robot model can be expressed as a nonlinear hybrid system containing two state manifolds (called "charts" in [44]):

$$
\begin{gathered}
\Sigma_{\mathrm{f}}:\left\{\begin{aligned}
\mathcal{X}_{\mathrm{f}} & =T \mathcal{Q}_{\mathrm{f}} \\
\mathcal{F}_{\mathrm{f}}:\left(\dot{x}_{\mathrm{f}}\right) & =f_{\mathrm{f}}\left(x_{\mathrm{f}}\right)+g_{\mathrm{f}}\left(x_{\mathrm{f}}\right) u \\
\mathcal{S}_{\mathrm{f}}^{\mathrm{s}} & =\left\{x_{\mathrm{f}} \in \mathcal{X}_{\mathrm{f}} \mid H_{\mathrm{f}}^{\mathrm{s}}\left(x_{\mathrm{f}}\right)=0\right\} \\
\mathcal{T}_{\mathrm{f}}^{\mathrm{s}}: x_{\mathrm{s}}^{+} & =\Delta_{\mathrm{f}}^{\mathrm{s}}\left(x_{\mathrm{f}}^{-}\right)
\end{aligned}\right. \\
\Sigma_{\mathrm{s}}:\left\{\begin{aligned}
\mathcal{X}_{\mathrm{s}} & =T \mathcal{Q}_{\mathrm{s}} \\
\mathcal{F}_{\mathrm{s}}:\left(\dot{x}_{\mathrm{s}}\right) & =f_{\mathrm{s}}\left(x_{\mathrm{s}}\right)+g_{\mathrm{s}}\left(x_{\mathrm{s}}\right) u \\
\mathcal{S}_{\mathrm{s}}^{\mathrm{f}} & =\left\{x_{\mathrm{s}} \in \mathcal{X}_{\mathrm{s}} \mid H_{\mathrm{s}}^{\mathrm{f}}\left(x_{\mathrm{s}}\right)=0\right\} \\
\mathcal{T}_{\mathrm{s}}^{\mathrm{f}}: x_{\mathrm{f}}^{+} & =\Delta_{\mathrm{s}}^{\mathrm{f}}\left(x_{\mathrm{s}}^{-}\right)
\end{aligned}\right.
\end{gathered}
$$

where, for example, $\mathcal{F}_{\mathrm{f}}$ is the flow on state manifold $\mathcal{X}_{\mathrm{f}}, \mathcal{S}_{\mathrm{f}}^{\mathrm{s}}$ is the switching hyper-surface for transitions between $\mathcal{X}_{\mathrm{f}}$ and $\mathcal{X}_{\mathrm{s}}$, and $\mathcal{T}_{\mathrm{f}}^{\mathrm{s}}: \mathcal{S}_{\mathrm{f}}^{\mathrm{s}} \rightarrow \mathcal{X}_{\mathrm{s}}$ is the transition function applied when $x_{\mathrm{f}} \in \mathcal{S}_{\mathrm{f}}^{\mathrm{s}}$.

The transition from flight phase to stance phase occurs when leg-2 impacts the ground. Hence, $H_{\mathrm{f}}^{\mathrm{s}}\left(x_{\mathrm{f}}\right)=\mathbf{y}_{2}$; see Fig. 1 . The ensuing initial value of the stance phase, $x_{\mathrm{s}}^{+}$, is determined from the impact model of Section III-D. A relabeling matrix $R$ is applied to the angular coordinates to account for the impact 
occurring on leg- 2 while the stance model assumes leg- 1 is in contact with the ground:

$$
\Delta_{\mathrm{f}}^{\mathrm{s}}\left(x_{\mathrm{f}}^{-}\right)=\left[\begin{array}{c}
{\left[R \mid 0_{5 \times 2}\right] q_{\mathrm{f}}^{-}} \\
R \tilde{\Delta}\left(x_{\mathrm{f}}^{-}\right)
\end{array}\right],
$$

and

$$
R=\left[\begin{array}{lllll}
0 & 0 & 1 & 0 & 0 \\
0 & 0 & 0 & 1 & 0 \\
1 & 0 & 0 & 0 & 0 \\
0 & 1 & 0 & 0 & 0 \\
0 & 0 & 0 & 0 & 1
\end{array}\right],
$$

where (21) has been used.

The transition from stance phase to flight phase can be initiated by causing the acceleration of the stance leg end to become positive. If torque discontinuities ${ }^{1}$ are allowedas they are assumed to be in this paper-when to transition into the flight phase becomes a control decision. Here, in view of simplifying the analysis of periodic orbits in Section VII, the transition is assumed to occur at a pre-determined point in the stance phase. Hence, $H_{\mathrm{s}}^{\mathrm{f}}=\theta_{\mathrm{s}}(q)-\theta_{\mathrm{s}, 0}^{-}$, where $\theta_{\mathrm{s}}(q):=$ $\frac{q_{1}}{2}+q_{2}+q_{5}$ is the angle ${ }^{2}$ of the hips with respect to end of the stance leg (see Fig. 1) and $\theta_{\mathrm{s}, 0}^{-}$is a constant to be determined. The ensuing initial value of the flight phase, $x_{f}^{+}$, is defined so as to achieve continuity in the position and velocity variables, using (8) and (9):

$$
\Delta_{\mathrm{s}}^{\mathrm{f}}\left(x_{\mathrm{s}}^{-}\right)=\left[\begin{array}{c}
{\left[\begin{array}{c}
q^{-} \\
\mathbf{f}_{1}\left(q^{-}\right)
\end{array}\right]} \\
{\left[\begin{array}{c}
q^{-} \\
\left.\frac{\partial \mathbf{f}_{1}}{\partial q}\right|_{q^{-}} \dot{q}^{-}
\end{array}\right]}
\end{array}\right] .
$$

Continuity of the torques is not imposed, and hence neither is continuity of the accelerations. It is assumed that the control law in the flight phase will be designed to achieve $\ddot{\mathbf{y}}_{1}^{\mathrm{f}+}>0$; see [11].

The definition of a solution of the hybrid model is adopted from [44]. For the definitions of orbital stability in the sense of Lyapunov, attractivity, and orbital asymptotic stability in the sense of Lyapunov, see [2], [45], [46].

\section{Qualitative Discussion of Control LaW Design}

This section describes in qualitative terms a control law design for planar bipedal running that is presented in detail in Section VI, analyzed in Section VII, and illustrated via simulations on RABBIT in Section VIII. A related discussion about control law design for walking is available in [1]. An important difference is that running has two continuous-time

\footnotetext{
${ }^{1}$ This is a modeling decision. In practice, the torque is continuous due to actuator dynamics. It is assumed here that the actuator time constant is small enough that it need not be modeled. See the very end of Section IX-B for further discussion of this point.

${ }^{2}$ In RABBIT the femur and tibia are of equal length. In general, any function that is independent of the body coordinates and is monotonically increasing along the motion of the robot can be used. As long as the tibia and femur are approximately of the same length, $\theta_{\mathrm{S}}(q):=\frac{q_{1}}{2}+q_{2}+q_{5}$ is a reasonable choice.
}

phases, stance and flight, and discrete transitions between them. Moreover, the flight phase presents more complications for controller design than does the stance phase: it has two additional degrees of freedom and angular momentum about the center of mass is conserved.

\section{A. Analytical tractability through invariance, attractivity, and configuration determinism at transitions}

Above all, the control strategy is constructed to facilitate the stability analysis of the closed-loop hybrid system consisting of the robot, the running surface, and the feedback controller. If stability analysis can be rendered sufficiently simple, then it becomes possible to explore efficiently a large set of asymptotically-stable running gaits in order to find one that meets additional performance objectives, such as minimum energy consumption per distance traveled for a given average speed, or minimum peak-actuator power demand.

The controller will be hybrid, with continuous-time feedback signals applied in stance and flight phases, and discrete (or event-based) updates of controller parameters performed at transitions between phases. The design of the controller uses two principles that are ubiquitous in non-hybrid systems, namely invariance and attractivity, with the notion of invariance being extended to hybrid systems so as to include the discrete transitions as well as the continuous flow of the Lagrangian dynamics. Hybrid invariance will lead to the creation of a low-dimensional hybrid subsystem of the fullorder closed-loop system. The low-order hybrid subsystem is called the Hybrid Zero Dynamics (HZD) of running. Attractivity will mean that trajectories of the full-order closed-loop system converge locally and sufficiently rapidly to those of the hybrid zero dynamics so that existence and stability of periodic running motions can be restricted to the study of the hybrid zero dynamics. The Poincaré return map for the hybrid zero dynamics will turn out to be one-dimensional. When transitions between phases in the hybrid zero dynamics occur at known configurations of the robot-this is called configuration determinism at transitions-it will turn out that the Poincaré return map can be computed in closed form, thereby rendering stability analysis of the closed-loop system tractable.

\section{B. Desired geometry of the closed-loop system}

The objective of the control law design is to achieve the internal structure of the closed-loop system that is depicted in Fig. 2. The vertical surfaces $\mathcal{S}_{\mathrm{s}}^{\mathrm{f}}$ and $\mathcal{S}_{\mathrm{f}}^{\mathrm{s}}$ represent the points in the state space where the transitions from stance to flight and from flight to stance occur, respectively; see (29). The horizontal surface on the left, $\mathcal{Z}_{\mathrm{s}}$, is created by the stancephase controller; it is designed to be invariant in the usual sense that if the system is initialized in $\mathcal{Z}_{\mathrm{s}}$, the solution of the stance-phase differential equation remains in $\mathcal{Z}_{\mathrm{s}}$ until it intersects $\mathcal{S}_{\mathrm{s}}^{\mathrm{f}}$, at which time the flight phase is initiated. The family of horizontal surfaces on the right, $\mathcal{Z}_{\mathrm{f}, a_{\mathrm{f}}}$, (shown for two values of $a_{\mathrm{f}}$, namely, $a_{\mathrm{f}, 1}$ and $\left.a_{\mathrm{f}, 4}\right)$ is created by the flightphase controller; each surface is designed to be invariant in the usual sense that if the system is initialized in $\mathcal{Z}_{\mathrm{f}, a_{\mathrm{f}}}$, the 


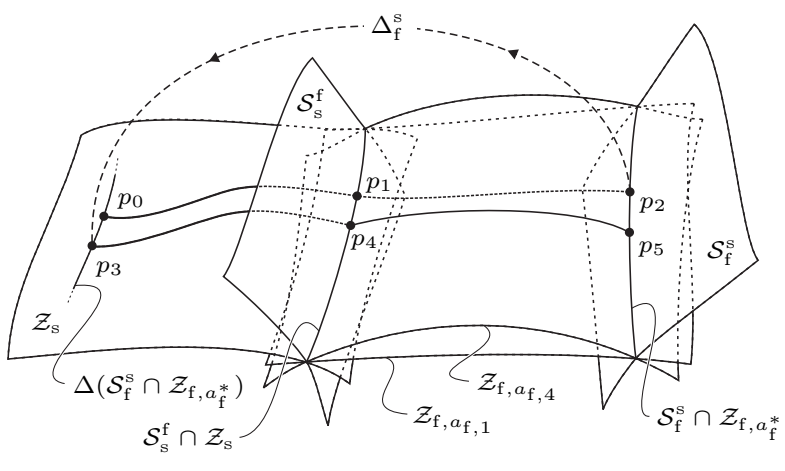

Fig. 2. Geometry of the closed-loop system that is achieved with the controller presented in this paper. Under the action of the feedback controller, the state of the system evolves on the low-dimensional surfaces $\mathcal{Z}_{\mathrm{s}}, \mathcal{Z}_{\mathrm{f}, a_{\mathrm{f}}}$. Consider two strides of a running cycle initiated at the beginning of stance (at $p_{0}$ ). The robot's state evolves in $\mathcal{Z}_{\mathrm{s}}$ throughout stance until the state enters $\mathcal{S}_{\mathrm{s}}^{\mathrm{f}}\left(\right.$ at $\left.p_{1}\right)$, when two discrete events occur: the robot lifts off to begin the flight phase; and the controller selects a value of $a_{\mathrm{f}}$ that will be held constant throughout the flight phase. The value of the parameter, call it $a_{\mathrm{f}, 1}$, is determined as a function of the angular momentum about the stance leg end at transition into flight in such a way that the robot will land in a pre-determined configuration. During the flight phase, the state of the robot evolves in $\mathcal{Z}_{\mathrm{f}, a_{\mathrm{f}, 1}}$ until it enters $\mathcal{S}_{\mathrm{f}}^{\mathrm{s}}\left(\right.$ at $\left.p_{2}\right)$. The impact mapping $\Delta_{\mathrm{f}}^{\mathrm{s}}$ is then applied and the next stance phase is begun (at $p_{3}$ ) and the process repeats. The robot will terminate the ensuing stance phase (at $p_{4}$ ) with an angular momentum that is different than what it had on the previous stride (at $p_{1}$ ). Due to conservation of angular momentum about the center of mass in the flight phase, the robot must evolve on a different surface in the flight phase this time in order to land in the same configuration it had on the previous landing. The required change in flight-phase evolution is accomplished by appropriate selection of $a_{\mathrm{f}, 4}$, which specifies $\mathcal{Z}_{\mathrm{f}, a_{\mathrm{f}, 4}}$. By design, the rule for selecting $a_{\mathrm{f}}$ results in $\mathcal{S}_{\mathrm{f}}^{\mathrm{s}} \cap \mathcal{Z}_{\mathrm{f}, a_{\mathrm{f}, 1}}=\mathcal{S}_{\mathrm{f}}^{\mathrm{s}} \cap \mathcal{Z}_{\mathrm{f}, a_{\mathrm{f}, 4}}$. This common set has been denoted by $\mathcal{S}_{\mathrm{f}}^{\mathrm{s}} \cap \mathcal{Z}_{\mathrm{f}, a_{\mathrm{f}}^{*}}$, where $a_{\mathrm{f}}^{*}$ is the parameter value corresponding to a periodic orbit.

solution of the flight-phase differential equation remains in $\mathcal{Z}_{\mathrm{f}, a_{\mathrm{f}}}$ until it intersects $\mathcal{S}_{\mathrm{f}}^{\mathrm{s}}$, at which time the stance phase is re-initialized through the impact map, $\Delta_{\mathrm{f}}^{\mathrm{s}}$. The value of the parameter $a_{\mathrm{f}}$ is selected at each transition from stance to flight as a function of the angular momentum of the robot about the stance leg end, $\sigma_{1}^{\mathrm{s}-}$, in such a way that evolution along the surface $\mathcal{Z}_{\mathrm{f}, a_{\mathrm{f}}\left(\sigma_{1}^{\mathrm{s}}\right)}$ will cause the robot to land on $\mathcal{Z}_{\mathrm{s}}$ with a pre-determined configuration. This brings out a second form of invariance that is related to the hybrid nature of the system: the parameter $a_{\mathrm{f}}$ is selected so that the composition of the flight-phase dynamics with the impact map $\Delta_{\mathrm{f}}^{\mathrm{s}}$ maps $\mathcal{Z}_{\mathrm{s}} \cap \mathcal{S}_{\mathrm{s}}^{\mathrm{f}}$ back to $\mathcal{Z}_{\mathrm{s}}$. This is shown in Fig. 2 with the arrow looping from the end of the flight phase back to the initiation of the stance phase, and more succinctly in Fig. 3, where the composition of the flight-phase dynamics with the impact map is represented by a "generalized" impact map, $\Delta$.

Not shown in either figure is the behavior of the robot off of the hybrid zero dynamics. This is where attractivity comes into play. In addition to creating the invariant surfaces, the feedback controller must also ensure that trajectories that start off of the surfaces converge to the surfaces. Both the creation of the invariant surfaces and their attractivity are accomplished with the use of virtual constraints.

\section{Virtual constraints}

Virtual constraints are holonomic constraints on the robot's configuration that are asymptotically imposed through feedback control. Their function is to coordinate the evolution

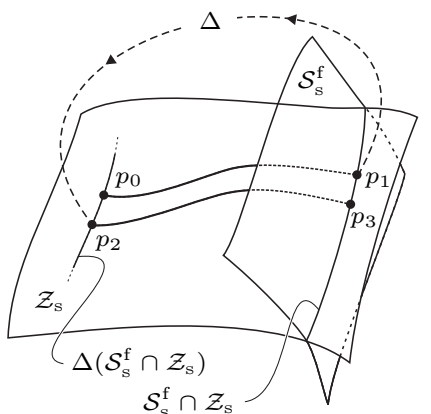

Fig. 3. Geometry of the closed-loop system when the flight phase and the impact map $\Delta_{\mathrm{f}}^{\mathrm{s}}$ are composed to form a generalized impact map $\Delta$ that maps $\mathcal{S}_{\mathrm{s}}^{\mathrm{f}} \cap \mathcal{Z}_{\mathrm{s}}$ to $\mathcal{Z}_{\mathrm{s}}$. This is analogous to the geometry that previous work has designed into walking. The analogy becomes exact if the robot's configuration at the initiation of the stance phase is the same for all points in $\mathcal{Z}_{\mathrm{s}} \cap \mathcal{S}_{\mathrm{S}}^{\mathrm{f}}$.

of the various links throughout a stride-which is another way of saying that they reduce the degrees of freedom. It is the imposition of the constraints that creates the invariant surfaces. Since RABBIT has four independent actuators (two at the hips and two and the knees), four virtual constraints may be imposed in both the stance and flight phases. The virtual constraints used in this paper are constructed as follows. Consider a function pair $\left\{\theta(q), h_{d}(\theta)\right\}$, where $\theta: \mathcal{Q} \rightarrow \mathbb{R}$ is a scalar function of the configuration variables (in stance or flight) and $h_{d}: \mathbb{R} \rightarrow \mathbb{R}^{4}$ gives the desired configuration of the actuated joints. $\theta(q)$ should be selected to be monotonic along the planned motion and, for purposes of analysis, it is required that $q_{\mathrm{b}}$ and $\theta$ be independent, that is, $\left[q_{\mathrm{b}}^{\prime}, \theta\right]^{\prime}$ is a valid change of coordinates on $\mathcal{Q}$. The virtual constraint is expressed in the form of an output $y=h(q):=q_{b}-h_{d} \circ \theta(q)$, and a controller is designed to asymptotically enforce the constraint by driving the output asymptotically to zero. Since the input and output have the same number of components, designing a controller to drive the output to zero is a standard problem [47]. The control action of driving the output to zero is what causes the trajectories of the system off of the invariant surface to converge to the surface.

\section{Control Law Development}

Separate state-variable control strategies are developed for the stance and flight phases of the running cycle. The controller for each phase is formulated as an output zeroing problem resulting in non-trivial zero dynamics [1], [3]. For the flight phase, it will be advantageous to allow the control to depend also on the initial value of the flight state or, equivalently, the final value of the stance state.

\section{A. Stance phase control}

As in $[3$, Sec. V], define the output

$$
y_{\mathrm{s}}=h_{\mathrm{s}}(q):=q_{\mathrm{b}}-h_{d, \mathrm{~s}} \circ \theta_{\mathrm{s}}(q)
$$

on (14), where the twice continuously differentiable function $h_{d, \mathrm{~s}}: \mathbb{R} \rightarrow \mathbb{R}^{4}$ encodes the stance-phase gait. It is assumed that the associated decoupling matrix ${ }^{3}, L_{g_{\mathrm{s}}} L_{f_{\mathrm{s}}} h_{\mathrm{s}}$, is invertible,

${ }^{3}$ The control law is rewritten without the use of Lie derivatives in Appendix I. 


$$
\begin{array}{r}
\Phi_{\mathrm{s}}(q):=\left[h_{\mathrm{s}}^{\prime}, \theta_{\mathrm{s}}\right]^{\prime} \text { is a global diffeomorphism on } \mathcal{Q}_{\mathrm{s}}, \\
\mathcal{Z}_{\mathrm{s}}:=\left\{x_{\mathrm{s}} \in T \mathcal{Q}_{\mathrm{s}} \mid h_{\mathrm{s}}\left(x_{\mathrm{s}}\right)=0, L_{f_{\mathrm{s}}} h_{\mathrm{s}}\left(x_{\mathrm{s}}\right)=0\right\}
\end{array}
$$

is an embedded two-dimensional submanifold of $T \mathcal{Q}_{\mathrm{s}}$, and $\mathcal{S}_{\mathrm{s}}^{\mathrm{f}} \cap \mathcal{Z}_{\mathrm{s}}$ is an embedded one-dimensional submanifold of $T \mathcal{Q}_{\mathrm{s}}$; see Fig. 2.

The feedback control is chosen to be continuous and to render $\mathcal{Z}_{\mathrm{s}}$ invariant under the closed-loop dynamics as well as attractive in finite time (the exact hypotheses from [3] are given in Appendix II):

$$
\begin{aligned}
u_{\mathrm{s}}\left(x_{\mathrm{s}}\right)=\left(L_{g_{\mathrm{s}}} L_{f_{\mathrm{s}}} h_{\mathrm{s}}\left(x_{\mathrm{s}}\right)\right)^{-1}\left(v \left(h_{\mathrm{s}}\left(x_{\mathrm{s}}\right)\right.\right. & \left., L_{f_{\mathrm{s}}} h_{\mathrm{s}}\left(x_{\mathrm{s}}\right)\right) \\
& \left.-L_{f_{\mathrm{s}}}^{2} h_{\mathrm{s}}\left(x_{\mathrm{s}}\right)\right),
\end{aligned}
$$

where $v$ renders the origin of

$$
\frac{d^{2} y_{\mathrm{s}}}{d t^{2}}=v
$$

globally asymptotically stable with finite convergence time. The closed-loop system is denoted

$$
f_{\mathrm{cl}, \mathrm{s}}\left(x_{\mathrm{s}}\right):=f_{\mathrm{s}}\left(x_{\mathrm{s}}\right)+g_{\mathrm{s}}\left(x_{\mathrm{s}}\right) u_{\mathrm{s}}\left(x_{\mathrm{s}}\right) .
$$

The feedback control

$$
u_{\mathrm{s}}^{*}\left(x_{\mathrm{s}}\right)=-\left(L_{g_{\mathrm{s}}} L_{f_{\mathrm{s}}} h_{\mathrm{s}}\left(x_{\mathrm{s}}\right)\right)^{-1} L_{f_{\mathrm{s}}}^{2} h_{\mathrm{s}}\left(x_{\mathrm{s}}\right)
$$

renders $\mathcal{Z}_{\mathrm{s}}$ invariant under the stance-phase dynamics; that is, for every $z \in \mathcal{Z}_{\mathrm{S}}$,

$$
f_{\text {zero }}(z):=f_{\mathrm{s}}(z)+g_{\mathrm{s}}(z) u_{\mathrm{s}}^{*}(z) \in T_{z} \mathcal{Z}_{\mathrm{s}} .
$$

$\mathcal{Z}_{\mathrm{s}}$ is called the stance-phase zero dynamics manifold and $\dot{z}=f_{\text {zero }}(z)$ is called the stance-phase zero dynamics. Following the development in [1], [3], $\left(\theta_{\mathrm{s}}, \sigma_{1}\right)$ is a valid set of local coordinates for $\mathcal{Z}_{\mathrm{s}}$ and in these coordinates the zero dynamics has the form

$$
\begin{aligned}
\dot{\theta}_{\mathrm{s}} & =\frac{1}{\mathbf{I}\left(\theta_{\mathrm{s}}\right)} \sigma_{1}, \\
\dot{\sigma}_{1} & =m g \mathbf{x}_{\mathrm{cm}}\left(\theta_{\mathrm{s}}\right),
\end{aligned}
$$

where $\mathbf{I}\left(\theta_{\mathbf{S}}\right)$ plays the role of an inertia. Moreover, in these coordinates, $\mathcal{S}_{\mathrm{s}}^{\mathrm{f}} \cap \mathcal{Z}_{\mathrm{s}}$ is given by

$$
\left\{\left(q_{0}^{\mathrm{s}-}, \dot{q}^{\mathrm{s}-}\right) \mid q_{0}^{\mathrm{s}-}=\Phi_{\mathrm{s}}^{-1}\left(0, \theta_{\mathrm{s}, 0}^{-}\right), \dot{q}^{\mathrm{s}-}=\dot{q}_{0}^{\mathrm{s}-} \sigma_{1}^{\mathrm{s}-}, \sigma_{1}^{\mathrm{s}-} \in \mathbb{R}\right\},
$$

where

$$
\dot{q}_{0}^{\mathrm{s}-}=\left.\left[\begin{array}{c}
\frac{\partial h_{\mathrm{s}}}{\partial q} \\
A_{5}
\end{array}\right]^{-1}\right|_{q_{0}^{\mathrm{s}-}}\left[\begin{array}{c}
0_{4 \times 1} \\
1
\end{array}\right] .
$$

For later use in computing a Poincaré return map on the zero dynamics, it is noted that (40) has Lagrangian [3, Eq. (59)] $\mathcal{L}_{\text {s,zero }}:=K_{\text {s,zero }}-V_{\text {s,zero }}$, where

$$
\begin{aligned}
K_{\mathrm{s}, \text { zero }} & :=\frac{1}{2}\left(\sigma_{1}\right)^{2} \\
V_{\mathrm{s}, \text { zero }}\left(\theta_{\mathrm{s}}\right) & :=-\int_{\theta_{\mathrm{s}}^{+}}^{\theta_{\mathrm{s}}} \mathbf{I}(\xi) m g \mathbf{x}_{\mathrm{cm}}(\xi) d \xi ;
\end{aligned}
$$

the choice of the lower limit $\theta_{\mathrm{s}}^{+}$is arbitrary and will be made later. Also for later use, define

$$
\left[\begin{array}{l}
\lambda_{x}\left(q_{0}^{\mathrm{s}-}\right) \\
\lambda_{y}\left(q_{0}^{\mathrm{s}-}\right)
\end{array}\right]:=\frac{\partial \mathbf{f}_{1}\left(q_{0}^{\mathrm{s}-}\right)}{\partial q} \dot{q}_{0}^{\mathrm{s}-},
$$

so that

$$
\left.\left[\begin{array}{c}
\dot{\mathbf{x}}_{\mathrm{cm}}^{\mathrm{s}-} \\
\dot{\mathbf{y}}_{\mathrm{cm}}^{\mathrm{s}-}
\end{array}\right]\right|_{\mathcal{S}_{\mathrm{s}}^{\mathrm{f} \cap \mathcal{Z}_{\mathrm{s}}}}=\left[\begin{array}{c}
\lambda_{x}\left(q_{0}^{\mathrm{s}-}\right) \\
\lambda_{y}\left(q_{0}^{\mathrm{s}-}\right)
\end{array}\right] \sigma_{1}^{\mathrm{s}-} .
$$

\section{B. Flight phase control}

The overall goal of the flight-phase controller is to land the robot in a favorable manner for continuing with the stance phase. It will turn out that a particularly interesting objective is the following: if the robot enters the flight phase from the stance-phase zero dynamics manifold, $\mathcal{Z}_{\mathrm{s}}$, control the robot so that it lands on $\mathcal{Z}_{\mathrm{s}}$ in a fixed configuration. The analytical motivation for this objective will be made clear in Section VII. The feasibility of landing in a fixed configuration will be illustrated in Section VIII with a feedback controller that depends on $x_{\mathrm{f}}$ and the final value of the state of the preceding stance phase. To realize such a controller as a state-variable feedback, the flight-state vector is augmented with dummy variables, $\dot{a}_{\mathrm{f}}=0$, whose values can be set at the transition from stance to flight. Let $a_{\mathrm{f}} \in \mathcal{A}:=\mathbb{R}^{p}, p \in \mathbb{N}$.

In other regards, paralleling the development of the stance phase controller ${ }^{4}$, define the output

$$
y_{\mathrm{f}}=h_{\mathrm{f}}\left(q_{\mathrm{f}}, a_{\mathrm{f}}\right):=q_{\mathrm{b}}-h_{d, \mathrm{f}}\left(\mathbf{x}_{\mathrm{cm}}, a_{\mathrm{f}}\right),
$$

where $h_{d, \mathrm{f}}$ is at least twice differentiable. Then the following can be easily shown: for any value of $a_{\mathrm{f}}$,

1) the decoupling matrix, $L_{g_{\mathrm{f}}} L_{f_{\mathrm{f}}} h_{\mathrm{f}}$, is everywhere invertible;

2) $\Phi_{\mathrm{f}}:=\left[h_{\mathrm{f}}^{\prime}, q_{5}, \mathbf{x}_{\mathrm{cm}}, \mathbf{y}_{\mathrm{cm}}\right]^{\prime}$ is a global diffeomorphism on $\mathcal{Q}_{\mathrm{f}}$;

3) the flight-phase zero-dynamics manifold

$$
\mathcal{Z}_{\mathrm{f}, a_{\mathrm{f}}}:=\left\{x_{\mathrm{f}} \in T \mathcal{Q}_{\mathrm{f}} \mid h_{\mathrm{f}}\left(x_{\mathrm{f}}, a_{\mathrm{f}}\right)=0, L_{f_{\mathrm{f}}} h_{\mathrm{f}}\left(x_{\mathrm{f}}, a_{\mathrm{f}}\right)=0\right\}
$$

is a six-dimensional embedded submanifold of $T \mathcal{Q}_{\mathrm{f}}$;

4) $\mathcal{S}_{\mathrm{f}}^{\mathrm{s}} \cap \mathcal{Z}_{\mathrm{f}, a_{\mathrm{f}}}$ is a five-dimensional embedded submanifold of $T \mathcal{Q}_{\mathrm{f}}$;

5) $\left(q_{5}, \mathbf{x}_{\mathrm{cm}}, \mathbf{y}_{\mathrm{cm}}, \sigma_{\mathrm{cm}}, \dot{\mathbf{x}}_{\mathrm{cm}}, \dot{\mathbf{y}}_{\mathrm{cm}}\right)$ is a set of global coordinates for $\mathcal{Z}_{\mathrm{f}, a_{\mathrm{f}}}$; and

6) the flight-phase zero dynamics has the form

$$
\begin{aligned}
\dot{q}_{5} & =\kappa_{1, \mathrm{f}}\left(\sigma_{\mathrm{cm}}, \mathbf{x}_{\mathrm{cm}}, \dot{\mathbf{x}}_{\mathrm{cm}}, a_{\mathrm{f}}\right) \\
\dot{\sigma}_{\mathrm{cm}} & =0 \\
\ddot{\mathbf{x}}_{\mathrm{cm}} & =0 \\
\ddot{\mathbf{y}}_{\mathrm{cm}} & =-g,
\end{aligned}
$$

where (49) arises from evaluating

$$
\dot{q}_{5}=\frac{\sigma_{\mathrm{cm}}}{A_{55}\left(q_{\mathrm{b}}\right)}-\sum_{i=1}^{4} \frac{A_{5 i}\left(q_{\mathrm{b}}\right)}{A_{55}\left(q_{\mathrm{b}}\right)} \dot{q}_{i}
$$

on $\mathcal{Z}_{\mathrm{f}, a_{\mathrm{f}}}$. Note that in Fig. 2, only a two-dimensional projection of $\mathcal{Z}_{\mathrm{f}, a_{\mathrm{f}}}$ could be shown and $\mathcal{S}_{\mathrm{f}}^{\mathrm{s}} \cap \mathcal{Z}_{\mathrm{f}, a_{\mathrm{f}}}$ was represented as a one-dimensional projection.

\footnotetext{
${ }^{4}$ As in the stance phase, the control law for the flight phase is rewritten without the use of Lie derivatives in Appendix I.
} 
The feedback controller is defined as

$$
\begin{aligned}
u_{\mathrm{f}}\left(x_{\mathrm{f}}, a_{\mathrm{f}}\right):=- & \left(L_{g_{\mathrm{f}}} L_{f_{\mathrm{f}}} h_{\mathrm{f}}\left(x_{\mathrm{f}}, a_{\mathrm{f}}\right)\right)^{-1}\left(K_{p} h_{\mathrm{f}}\left(x_{\mathrm{f}}, a_{\mathrm{f}}\right)\right. \\
& \left.+K_{d} L_{f_{\mathrm{f}}} h_{\mathrm{f}}\left(x_{\mathrm{f}}, a_{\mathrm{f}}\right)+L_{f_{\mathrm{f}}}^{2} h_{\mathrm{f}}\left(x_{\mathrm{f}}, a_{\mathrm{f}}\right)\right),
\end{aligned}
$$

where $\ddot{y}_{\mathrm{f}}+K_{d} \dot{y}_{\mathrm{f}}+K_{p} y_{\mathrm{f}}=0$ is exponentially stable. Let $\bar{x}_{\mathrm{f}}:=\left(x_{\mathrm{f}}^{\prime}, a_{\mathrm{f}}^{\prime}\right)^{\prime}$ and denote the right-hand side of (7) and the trivial parameter dynamics $\dot{a}_{\mathrm{f}}=0$ in closed loop with (54) by

$$
f_{\mathrm{cl}, \mathrm{f}}\left(\bar{x}_{\mathrm{f}}\right):=\left[\begin{array}{l}
f_{\mathrm{f}}\left(x_{\mathrm{f}}\right)+g_{\mathrm{f}}\left(x_{\mathrm{f}}\right) u_{\mathrm{f}}\left(\bar{x}_{\mathrm{f}}\right) \\
0
\end{array}\right] \text {. }
$$

\section{Closed-loop hybrid model}

The closed-loop hybrid model is

$$
\begin{aligned}
& \Sigma_{\mathrm{f}, c l}:\left\{\begin{aligned}
\overline{\mathcal{X}}_{\mathrm{f}} & =T \mathcal{Q}_{\mathrm{f}} \times \mathcal{A} \\
\overline{\mathcal{F}}_{\mathrm{cl}, \mathrm{f}}:\left(\dot{\bar{x}}_{\mathrm{f}}\right) & =f_{\mathrm{cl}, \mathrm{f}}\left(\bar{x}_{\mathrm{f}}\right) \\
\overline{\mathcal{S}}_{\mathrm{f}}^{\mathrm{s}} & =\left\{\left(x_{\mathrm{f}}, a_{\mathrm{f}}\right) \in \overline{\mathcal{X}}_{\mathrm{f}} \mid H_{\mathrm{f}}^{\mathrm{s}}\left(x_{\mathrm{f}}\right)=0\right\} \\
\overline{\mathcal{T}}_{\mathrm{f}}^{\mathrm{s}}: x_{\mathrm{s}}^{+} & =\bar{\Delta}_{\mathrm{f}}^{\mathrm{s}}\left(\bar{x}_{\mathrm{f}}^{-}\right):=\Delta_{\mathrm{f}}^{\mathrm{s}}\left(x_{\mathrm{f}}^{-}\right)
\end{aligned}\right. \\
& \Sigma_{\mathrm{s}, c l}:\left\{\begin{aligned}
\mathcal{X}_{\mathrm{s}} & =T \mathcal{Q}_{\mathrm{s}} \\
\mathcal{F}_{\mathrm{cl}, \mathrm{s}}:\left(\dot{x}_{\mathrm{s}}\right) & =f_{\mathrm{cl}, \mathrm{s}}\left(x_{\mathrm{s}}\right) \\
\mathcal{S}_{\mathrm{s}}^{\mathrm{f}} & =\left\{x_{\mathrm{s}} \in \mathcal{X}_{\mathrm{s}} \mid H_{\mathrm{s}}^{\mathrm{f}}\left(x_{\mathrm{s}}\right)=0\right\} \\
\overline{\mathcal{T}}_{\mathrm{s}}^{\mathrm{f}}: x_{\mathrm{f}}^{+} & =\Delta_{\mathrm{s}}^{\mathrm{f}}\left(x_{\mathrm{s}}^{-}\right), a_{\mathrm{f}}^{+}=w_{\mathrm{s}}^{\mathrm{f}}\left(x_{\mathrm{s}}^{-}\right),
\end{aligned}\right.
\end{aligned}
$$

where the parameter update law $w_{\mathrm{s}}^{\mathrm{f}}$ is at least continuously differentiable. The internal geometry of the closed-loop system is shown in Fig. 2.

\section{EXISTENCE AND StABILITy OF PERIOdic ORbits}

This section of the paper could equally well be titled, "Main Results", as it is here that the internal geometric structure of the closed-loop system (56) is exploited to obtain a lowdimensional, closed-form characterization of asymptotically stable, periodic, running motions. Section VII-A develops the Poincaré section and the Poincaré return map that will be used for analyzing periodic orbits of (56). The analytical results based on restriction dynamics-that is, the hybrid zero dynamics of Fig. 3-are developed in Section VII-B. The relation to stability in the full-order model is treated in Section VII-C.

\section{A. Definition of the Poincaré return map}

A periodic running motion corresponds to a periodic orbit in the closed-loop model. The Poincaré return map is a well known tool for determining the existence of periodic orbits and their stability properties; for its use in hybrid systems, see [2], [45], [48], [49]. A fixed point of the Poincaré return map is equivalent to a periodic orbit.

Since running consists of two phases, the Poincaré return map is naturally viewed as the composition of two maps: $P:=$ $P_{\mathrm{s}} \circ P_{\mathrm{f}}$, as in Fig. 4, where $P_{\mathrm{s}}: \overline{\mathcal{S}}_{\mathrm{f}}^{\mathrm{s}} \rightarrow \mathcal{S}_{\mathrm{s}}^{\mathrm{f}}$ follows a solution of the closed-loop model from the impact event at the end of flight to just before the end of stance, and $P_{\mathrm{f}}: \mathcal{S}_{\mathrm{s}}^{\mathrm{f}} \rightarrow \overline{\mathcal{S}}_{\mathrm{f}}^{\mathrm{s}}$ follows a solution from the end-of-stance event to just before the end of flight. Since not every landing will result in the

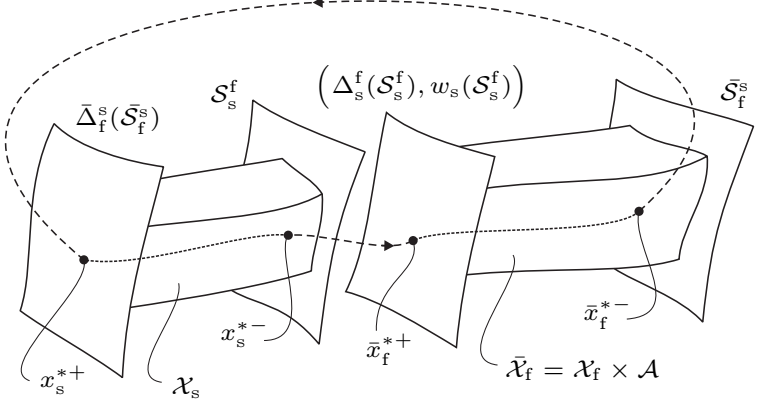

Fig. 4. Poincaré maps for the closed-loop system. Conceptually, $P_{\mathrm{s}}: \overline{\mathcal{S}}_{\mathrm{f}}^{\mathrm{s}} \rightarrow$ $\mathcal{S}_{\mathrm{S}}^{\mathrm{f}}$, and is determined by following the flow of the closed-loop stance model from the impact at the end of flight up to, and not including, the transition from stance to flight. Similarly, $P_{\mathrm{f}}: \mathcal{S}_{\mathrm{s}}^{\mathrm{f}} \rightarrow \overline{\mathcal{S}}_{\mathrm{f}}^{\mathrm{s}}$, and is determined by following the flow of the closed-loop flight model augmented with the trivial parameter dynamics $\left(\dot{a}_{\mathrm{f}}=0\right)$ from the transition at the end of stance up to, and not including, the impact event at end of flight. The Poincare map is $P: \mathcal{S}_{\mathrm{s}}^{\mathrm{f}} \rightarrow$ $\mathcal{S}_{\mathrm{s}}^{\mathrm{f}}$, where $P:=P_{\mathrm{s}} \circ P_{\mathrm{f}}$. A periodic orbit corresponds to a fixed point of $P$, namely, $x_{\mathrm{s}}^{*-}=P\left(x_{\mathrm{s}}^{*-}\right)$.

completion of a stance phase (for example, the robot may not have sufficient speed), $P_{\mathrm{S}}$ is only a partial map. And, since not every transition out of stance results in a successful flight phase followed by a successful stance phase, $P_{\mathrm{f}}$ is a partial map. Hence, the domains where these maps are well defined must be identified, which will give rise to the subsets $\tilde{\mathcal{S}}_{\mathrm{s}}^{\mathrm{f}} \subset \mathcal{S}_{\mathrm{S}}^{\mathrm{f}}$ and $\tilde{\mathcal{S}}_{\text {f }}^{\mathrm{s}} \subset \overline{\mathcal{S}}_{\text {f }}^{\text {s }}$.

Following [2], define the stance-time-to-impact function ${ }^{5}$, $T_{I, \mathrm{~s}}: x_{0} \in \mathcal{X}_{\mathrm{s}} \rightarrow \mathbb{R} \cup\{\infty\}$, by

$T_{I, \mathrm{~s}}:= \begin{cases}\inf \left\{t \geq 0 \mid \varphi_{\mathrm{cl}, \mathrm{s}}\left(t, x_{0}\right) \in \mathcal{S}_{\mathrm{s}}^{\mathrm{f}}\right\} & \text { if } \exists t \text { such that } \\ & \varphi_{\mathrm{cl}, \mathrm{s}}\left(t, x_{0}\right) \in \mathcal{S}_{\mathrm{s}}^{\mathrm{f}} \\ \infty & \text { otherwise, }\end{cases}$

where $\varphi_{\mathrm{cl}, \mathrm{s}}\left(t, x_{0}\right)$ is an integral curve of (37) corresponding to $\varphi_{\mathrm{cl}, \mathrm{s}}\left(0, x_{0}\right)=x_{0}$. ¿From [2, Lemma 3], $T_{I, \mathrm{~s}}$ is continuous at points $x_{0}$ where $0<T_{I, \mathrm{~s}}\left(x_{0}\right)<\infty$ and the intersection with $\mathcal{S}_{\mathrm{s}}^{\mathrm{f}}$ is transversal ${ }^{6}$. Hence, $\tilde{\mathcal{X}}_{\mathrm{s}}:=\left\{x_{\mathrm{s}} \in \mathcal{X}_{\mathrm{s}} \mid 0<\right.$ $\left.T_{I, \mathrm{~s}}\left(x_{\mathrm{s}}\right)<\infty, L_{f_{\mathrm{cl}, \mathrm{s}}} H_{\mathrm{s}}^{\mathrm{f}}\left(\varphi_{\mathrm{cl}, \mathrm{s}}\left(T_{I, \mathrm{~s}}\left(x_{\mathrm{s}}\right), x_{\mathrm{s}}\right)\right) \neq 0\right\}$ is open, and consequently, $\tilde{\mathcal{S}}_{\mathrm{f}}^{\mathrm{s}}:=\bar{\Delta}_{\mathrm{f}}^{\mathrm{s}}-1\left(\tilde{\mathcal{X}}_{\mathrm{s}}\right)$ is an open subset of $\overline{\mathcal{S}}_{\mathrm{f}}^{\mathrm{s}}$. It follows that the generalized Poincaré stance map $P_{\mathrm{s}}: \tilde{\mathcal{S}}_{\mathrm{f}}^{\mathrm{s}} \rightarrow$ $\mathcal{S}_{\mathrm{S}}^{\mathrm{f}}$ defined by

$$
P_{\mathrm{s}}\left(\bar{x}_{\mathrm{f}}\right):=\varphi_{\mathrm{cl}, \mathrm{s}}\left(T_{I, \mathrm{~s}}\left(\bar{\Delta}_{\mathrm{f}}^{\mathrm{s}}\left(\bar{x}_{\mathrm{f}}\right)\right), \bar{\Delta}_{\mathrm{f}}^{\mathrm{s}}\left(\bar{x}_{\mathrm{f}}\right)\right)
$$

is well defined and continuous (the terminology of a generalized-Poincaré map follows Appendix D of [50]).

Similarly, the generalized Poincaré flight map $P_{\mathrm{f}}: \tilde{\mathcal{S}}_{\mathrm{s}}^{\mathrm{f}} \rightarrow$ $\tilde{\mathcal{S}}_{\mathrm{f}}^{\mathrm{s}}$, is defined by

$$
P_{\mathrm{f}}\left(x_{\mathrm{s}}\right):=\varphi_{\mathrm{cl}, \mathrm{f}}\left(T_{I, \mathrm{f}}\left(\Delta_{\mathrm{s}}^{\mathrm{f}}\left(x_{\mathrm{s}}\right), w_{\mathrm{s}}^{\mathrm{f}}\left(x_{\mathrm{s}}\right)\right), \Delta_{\mathrm{s}}^{\mathrm{f}}\left(x_{\mathrm{s}}\right), w_{\mathrm{s}}^{\mathrm{f}}\left(x_{\mathrm{s}}\right)\right),
$$

${ }^{5}$ Flows from one surface to another are sometimes called impact maps or impact functions. $T_{I, \mathrm{~s}}$ could also be called the time-to-flight function.

${ }^{6}$ Transversality guarantees nonzero vertical leg end velocity at impact; that is, the foot does not just scuff the ground. 
where, $T_{I, \mathrm{f}}: \bar{x}_{0} \in \overline{\mathcal{X}}_{\mathrm{f}} \rightarrow \mathbb{R} \cup\{\infty\}$ by

$T_{I, \mathrm{f}}:= \begin{cases}\inf \left\{t \geq 0 \mid \varphi_{\mathrm{cl}, \mathrm{f}}\left(t, \bar{x}_{0}\right) \in \tilde{\mathcal{S}}_{\mathrm{f}}^{\mathrm{s}}\right\} & \text { if } \exists t \text { such that } \\ \infty & \varphi_{\mathrm{cl}, \mathrm{f}}\left(t, \bar{x}_{0}\right) \in \tilde{\mathcal{S}}_{\mathrm{f}}^{\mathrm{s}} \\ & \text { otherwise, }\end{cases}$

and $\tilde{\mathcal{S}}_{\mathrm{s}}^{\mathrm{f}}=\left\{x_{\mathrm{s}} \in \mathcal{S}_{\mathrm{s}}^{\mathrm{f}} \mid 0<T_{I, \mathrm{f}}\left(\Delta_{\mathrm{s}}^{\mathrm{f}}\left(x_{\mathrm{s}}\right), w_{\mathrm{s}}^{\mathrm{f}}\left(x_{\mathrm{s}}\right)\right)<\right.$ $\infty, L_{f_{\mathrm{cl}, \mathrm{f}}} H_{\mathrm{f}}^{\mathrm{s}}\left(\varphi_{\mathrm{cl}, \mathrm{f}}\left(T_{I, \mathrm{f}}\left(\Delta_{\mathrm{s}}^{\mathrm{f}}\left(x_{\mathrm{s}}\right), w_{\mathrm{s}}^{\mathrm{f}}\left(x_{\mathrm{s}}\right)\right), \Delta_{\mathrm{s}}^{\mathrm{f}}\left(x_{\mathrm{s}}\right), w_{\mathrm{s}}^{\mathrm{f}}\left(x_{\mathrm{s}}\right)\right)\right) \neq$ $0\}$. In [50, Appendix D], it is proved that $P_{\mathrm{f}}$ is continuously differentiable.

The Poincaré return map $P: \tilde{\mathcal{S}}_{\mathrm{s}}^{\mathrm{f}} \rightarrow \mathcal{S}_{\mathrm{s}}^{\mathrm{f}}$ for (56) is defined by

$$
P:=P_{\mathrm{s}} \circ P_{\mathrm{f}}
$$

\section{B. Analysis of the Poincare return map}

Theorem 1 (Connecting running to walking): Let $P$ be as (61), and let $\mathcal{S}:=\mathcal{S}_{\mathrm{s}}^{\mathrm{f}}$, and $\tilde{\mathcal{S}}:=\tilde{\mathcal{S}}_{\mathrm{s}}^{\mathrm{f}}$. Then $P$ is also the Poincaré return map for the system with impulse effects

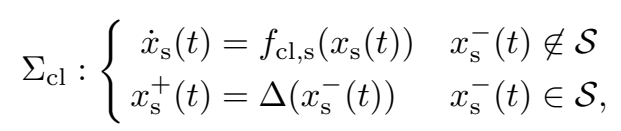

where $\Delta:=\bar{\Delta}_{\mathrm{f}}^{\mathrm{s}} \circ P_{\mathrm{f}}$.

Proof: This follows from the construction of the Poincaré return map in [2, Eq. (14)].

This observation is important because models of the form (62)—called systems with impulse effects [51], [52]— have been studied in the context of walking gaits [3], [4]. The association of running with walking may indicate how results developed for walking, such as closed-form stability analysis on the basis of a restricted Poincaré map, may be extended to running. One motivation for pursuing this association is the fact that the control law designs of [3], [4] have been validated experimentally [8], [9]. In this section and the next, several results along this line of reasoning are developed and illustrated on an asymptotically stable running gait.

Suppose that $\Delta\left(\tilde{\mathcal{S}} \cap \mathcal{Z}_{\mathrm{S}}\right) \subset \mathcal{Z}_{\mathrm{s}}$, where $\mathcal{Z}_{\mathrm{s}}$ is the stance-phase zero dynamics manifold. Then, from [3], (62) has a hybrid zero dynamics, which may be called the hybrid zero dynamics of running:

$$
\begin{aligned}
\dot{z} & =f_{\text {zero }}(z) & & z^{-} \notin \mathcal{S} \cap \mathcal{Z}_{\mathrm{s}} \\
z^{+} & =\Delta_{\text {zero }}\left(z^{-}\right) & & z^{-} \in \mathcal{S} \cap \mathcal{Z}_{\mathrm{s}},
\end{aligned}
$$

where the restricted impact map is $\Delta_{\text {zero }}:=\left.\Delta\right|_{\tilde{\mathcal{S}} \cap \mathcal{Z}_{\mathrm{s}}}$ and $f_{\text {zero }}$ is given by (39). The key properties in walking gaits that led to a rich analytic theory were $\mathcal{Z}_{\mathrm{s}}$-invariance, $\Delta\left(\tilde{\mathcal{S}} \cap \mathcal{Z}_{\mathrm{s}}\right) \subset \mathcal{Z}_{\mathrm{s}}$, and what one may call configuration determinism at transition: $\pi \circ \Delta\left(\tilde{\mathcal{S}} \cap \mathcal{Z}_{\mathrm{s}}\right)$ consists of a single point, where $\pi: T \mathcal{Q}_{\mathrm{s}} \rightarrow \mathcal{Q}_{\mathrm{s}}$ is the canonical projection. How to achieve these conditions for $\Delta=\bar{\Delta}_{\mathrm{f}}^{\mathrm{s}} \circ P_{\mathrm{f}}$ through design of the flight-phase controller will be detailed in Section VIII.

Let $q_{0}^{\mathrm{s}-}$ be as defined in (41) and define $q_{0}^{\mathrm{s}+}:=\pi \circ$ $\Delta\left(q_{0}^{\mathrm{s}-}, *\right)$. Use (8) to define the positions of the center of mass at the beginning of the stance phase, $\left(\mathbf{x}_{\mathrm{cm}}^{\mathrm{s}+}, \mathbf{y}_{\mathrm{cm}}^{\mathrm{s}+}\right)$, and the end of the stance phase, $\left(\mathbf{x}_{\mathrm{cm}}^{\mathrm{s}-}, \mathbf{y}_{\mathrm{cm}}^{\mathrm{s}-}\right)$. In the following, it is assumed that the center of mass is behind the stance leg at the beginning of the stance phase, and thus, $\mathbf{x}_{\mathrm{cm}}^{\mathrm{s}+}<0$.
Theorem 2 (Characterization of restricted impact map): Suppose that $\Delta\left(\tilde{\mathcal{S}} \cap \mathcal{Z}_{\mathrm{S}}\right) \subset \mathcal{Z}_{\mathrm{s}}$ and $\pi \circ \Delta\left(\tilde{\mathcal{S}} \cap \mathcal{Z}_{\mathrm{s}}\right)=\left\{q_{0}^{\mathrm{s}+}\right\}$. In the coordinates $\left(\theta_{\mathrm{s}}, \sigma_{1}\right)$ for $\mathcal{Z}_{\mathrm{s}}$, the restricted impact map is given by

$$
\Delta_{\text {zero }}\left(\theta_{\mathrm{s}, 0}^{-}, \sigma_{1}^{\mathrm{s}-}\right)=\left[\begin{array}{c}
\theta_{\mathrm{s}, 0}^{+} \\
\delta\left(\sigma_{1}^{\mathrm{s}-}\right)
\end{array}\right]
$$

where

$$
\begin{aligned}
\theta_{\mathrm{s}, 0}^{+} & =\theta_{\mathrm{s}}\left(q_{0}^{\mathrm{s}+}\right) \\
\delta\left(\sigma_{1}^{\mathrm{s}-}\right) & =\chi \sigma_{1}^{\mathrm{s}-}-\sqrt{\left(\beta \sigma_{1}^{\mathrm{s}-}\right)^{2}+\alpha}
\end{aligned}
$$

and

$$
\begin{aligned}
\alpha & =-2 m^{2} g\left(\mathbf{x}_{\mathrm{cm}}^{\mathrm{s}+}\right)^{2}\left(\mathbf{y}_{\mathrm{cm}}^{\mathrm{s}+}-\mathbf{y}_{\mathrm{cm}}^{\mathrm{s}-}\right) \\
\beta & =m \mathbf{x}_{\mathrm{cm}}^{\mathrm{s}+} \lambda_{y}\left(q_{0}^{\mathrm{s}-}\right) \\
\chi & =1+m \mathbf{x}_{\mathrm{cm}}^{\mathrm{s}-} \lambda_{y}\left(q_{0}^{\mathrm{s}-}\right)+m\left(\mathbf{y}_{\mathrm{cm}}^{\mathrm{s}+}-\mathbf{y}_{\mathrm{cm}}^{\mathrm{s}-}\right) \lambda_{x}\left(q_{0}^{\mathrm{s}-}\right) .
\end{aligned}
$$

The proof is given in Appendix III.

\section{Remark 4:}

1) $\chi<0$ would imply a sign change in the angular momentum at impact, which would be incompatible with the definition of running as forward motion with a flight phase.

2) If $\mathrm{x}_{\mathrm{cm}}^{\mathrm{s}+}<0$ is not assumed, the general expression for $(65)$ is $\delta\left(\sigma_{1}^{\mathrm{s}-}\right)=\chi \sigma_{1}^{\mathrm{s}-}+\operatorname{sgn}\left(\mathbf{x}_{\mathrm{cm}}^{\mathrm{s}+}\right) \sqrt{\left(\beta \sigma_{1}^{\mathrm{s}-}\right)^{2}+\alpha}$, where $\operatorname{sgn}(x)$ is the sign of $x$.

3) When $\alpha=0$, that is, the center of mass has the same height at the beginning and end of the stance phase, $\delta\left(\sigma_{1}^{\mathrm{s}-}\right)=(\chi-|\beta|) \sigma_{1}^{\mathrm{s}-}$ is linear, exactly as in walking.

4) In terms of the coordinates $\left(\theta_{\mathrm{s}, 0}^{-}, \zeta:=\frac{1}{2}\left(\sigma_{1}^{\mathrm{s}-}\right)^{2}\right)$ for $\tilde{\mathcal{S}} \cap \mathcal{Z}_{\mathrm{s}}$, where the (generalized) kinetic energy of the stance-phase zero dynamics is used instead of the angular momentum, the second entry in (64) becomes

$$
\begin{aligned}
& \delta_{e}(\zeta)=\left(\chi^{2}+\beta^{2}\right) \zeta \\
& -\chi \sqrt{2 \alpha \zeta+(2 \beta \zeta)^{2}}+\frac{\alpha}{2} .
\end{aligned}
$$

5) Implicit in the construction of $\tilde{\mathcal{S}}$ is the condition $2 \alpha \zeta+$ $(2 \beta \zeta)^{2} \geq 0$. Also a part of the construction of $\tilde{\mathcal{S}}$ is the condition that $T_{I, \mathrm{f}}$ is a positive real number; under the assumptions made on $\Delta$, this is equivalent to checking that $\mathbf{y}_{\mathrm{cm}}^{\mathrm{s}+}>\mathbf{y}_{\mathrm{cm}}^{\mathrm{s}-}$ and $\lambda_{y}\left(q_{0}^{\mathrm{s}-}\right)<0$ do not simultaneously occur.

Let $P: \tilde{\mathcal{S}} \rightarrow \mathcal{S}$ be the Poincaré return map for (62), and hence, also for (56), and suppose that $\Delta\left(\tilde{\mathcal{S}} \cap \mathcal{Z}_{\mathrm{s}}\right) \subset \mathcal{Z}_{\mathrm{s}}$, as in Fig. 3. Then $P\left(\tilde{\mathcal{S}} \cap \mathcal{Z}_{\mathrm{s}}\right) \subset \mathcal{S} \cap \mathcal{Z}_{\mathrm{s}}$, and the restriction map $\rho: \tilde{\mathcal{S}} \cap \mathcal{Z}_{\mathrm{s}} \rightarrow \mathcal{S} \cap \mathcal{Z}_{\mathrm{s}}$ by

$$
\rho:=\left.P\right|_{\tilde{\mathcal{S}} \cap \mathcal{Z}_{\mathrm{s}}}
$$

is well defined. The restricted Poincaré return map $\rho$ is important because it is scalar and, by [2, Theorem 2] (see [3, Sec. IV]), asymptotically stable fixed points of it correspond to asymptotically stable periodic orbits of the hybrid model (62), and hence, to asymptotically stable running gaits.

Theorem 3 (Closed-form for $\rho)$ : Suppose that $\Delta(\tilde{\mathcal{S}} \cap$ $\left.\mathcal{Z}_{\mathrm{s}}\right) \subset \mathcal{Z}_{\mathrm{s}}$ and $\pi \circ \Delta\left(\tilde{\mathcal{S}} \cap \mathcal{Z}_{\mathrm{s}}\right)=\left\{q_{0}^{\mathrm{s}+}\right\}$. Let $\left(\theta_{\mathrm{s}, 0}^{-}, \sigma_{1}^{\mathrm{s}-}\right) \in \tilde{\mathcal{S}} \cap \mathcal{Z}_{\mathrm{s}}$, and set $\zeta:=\frac{1}{2}\left(\sigma_{1}^{\mathrm{s}-}\right)^{2}$. Then

$$
\rho(\zeta)=\delta_{e}(\zeta)-V_{\mathrm{s}, \mathrm{zero}}\left(\theta_{\mathrm{s}, 0}^{-}\right),
$$




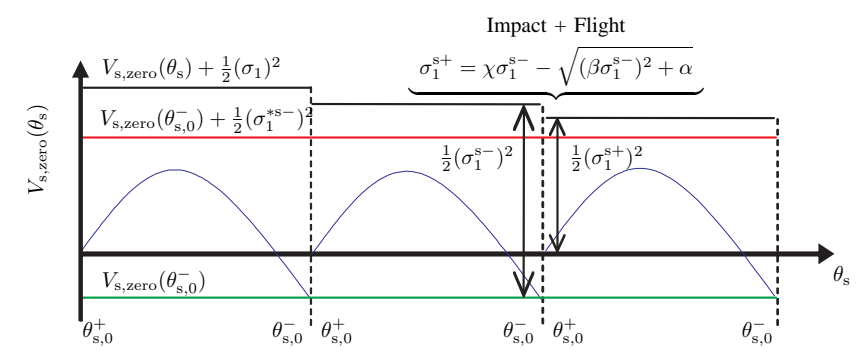

Fig. 5. The stance phase zero dynamics is Lagrangian, and thus throughout the stance phase, the corresponding total energy $V_{\mathrm{s}, \text { zero }}\left(\theta_{\mathrm{S}}\right)+\frac{1}{2}\left(\sigma_{1}\right)^{2}$ is constant. Over the impact plus flight phase, the change in total energy depends on the angular momentum through $\delta\left(\sigma_{1}^{\mathrm{s}-}\right)$ and the potential energy through $V_{\mathrm{s} \text {, zero }}\left(\theta_{\mathrm{s}, 0}^{-}\right)$. The total energy corresponding to the periodic orbit is $V_{\mathrm{s}, \text { zero }}\left(\theta_{\mathrm{s}, 0}^{-}\right)+\frac{1}{2}\left(\sigma_{1}^{* \mathrm{~s}-}\right)^{2}$.

with domain of definition

$$
\begin{aligned}
\mathcal{D}_{\rho}:=\left\{\zeta>0 \mid \delta_{e}(\zeta)-V_{\mathrm{s}, \text { zero }}^{\max }\right. & >0, \\
& \left.2 \alpha \zeta+(2 \beta \zeta)^{2} \geq 0\right\},
\end{aligned}
$$

where $\delta_{e}$ is defined in (67), and

$$
V_{\mathrm{s}, \mathrm{zero}}^{\max }:=\max _{\theta_{\mathrm{s}, 0}^{+} \leq \theta_{\mathrm{s}} \leq \theta_{\mathrm{s}, 0}^{-}} V_{\mathrm{s}, \mathrm{zero}}\left(\theta_{\mathrm{s}}\right) .
$$

Moreover, the first derivative of the restricted Poincaré return map is

$$
\frac{d \rho}{d \zeta}(\zeta)=\frac{d \delta_{e}}{d \zeta}(\zeta)=\left(\chi^{2}+\beta^{2}\right)-\chi \frac{\alpha+4 \beta^{2} \zeta}{\sqrt{2 \alpha \zeta+(2 \beta \zeta)^{2}}} .
$$

The proof is given in Appendix IV.

Remark 5: 1) Computing a fixed point of (69) is easily reduced to solving a quadratic equation. If its discriminate $\Upsilon$ is non-negative, where

$$
\begin{aligned}
& \Upsilon:=4 \chi^{2}\left(\chi^{2} \alpha^{2}\right. \\
& \left.+\left(-2 V_{\mathrm{s}, \text { zero }}\left(\theta_{\mathrm{s}, 0}^{-}\right)+\alpha\right)\left(-\alpha \chi^{2}+\alpha-2 \beta^{2} V_{\mathrm{s}, \text { zero }}\left(\theta_{\mathrm{s}, 0}^{-}\right)\right)\right),
\end{aligned}
$$

the fixed point can be explicitly calculated as

$$
\zeta^{*}=\frac{\left(\chi^{2}+\beta^{2}-1\right)\left(2 V_{\mathrm{s}, \text { zero }}\left(\theta_{\mathrm{s}, 0}^{-}\right)-\alpha\right)+2 \chi^{2} \alpha-\sqrt{\Upsilon}}{2\left((\chi+\beta)^{2}-1\right)\left((\chi-\beta)^{2}-1\right)}
$$

2) As in walking [1, Fig. 14], the restricted Poincaré map can be interpreted in terms of energy transfer; see Fig. 5.

The following two corollaries are immediate.

Corollary 1 (Exponentially stable fixed points):

Suppose that $\zeta^{*} \in \mathcal{D}_{\rho}$ is a fixed point of $\rho$. Then it is exponentially stable if, and only if,

$$
\mu:=\left(\chi^{2}+\beta^{2}\right)-\chi \frac{\alpha+4 \beta^{2} \zeta^{*}}{\sqrt{2 \alpha \zeta^{*}+\left(2 \beta \zeta^{*}\right)^{2}}}
$$

satisfies $|\mu|<1$.

Corollary 2 (Qualitative analysis of $\rho$ ): The following statements are true:

(a) $\lim _{\zeta \searrow 0} \frac{d \rho}{d \zeta}(\zeta)=-\infty$, for $\chi>0$ and $\alpha \geq 0$;

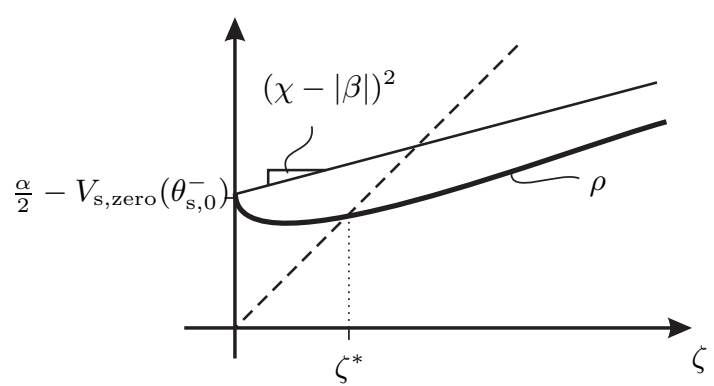

(a) stable

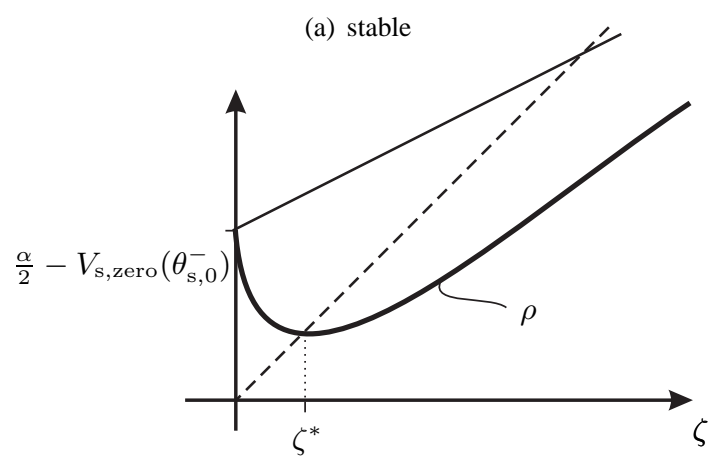

(b) unstable

Fig. 6. Qualitatively different Poincaré maps that may occur in running. The dashed line is the identity map and the bold line is a sketch of the restricted Poincaré return map. In (a), the fixed point is exponentially stable because the intersection with the identity line occurs with a positive slope less than 1.0. In (b), the fixed point is unstable because the intersection with the identity line occurs with a negative slope less than -1.0 .

(b) $\lim _{\zeta \searrow \frac{-\alpha}{2 \beta^{2}}} \frac{d \rho}{d \zeta}(\zeta)=-\infty$, for $\chi>0$ and $\alpha<0$;

(c) $\lim _{\zeta \rightarrow \infty} \frac{d \rho}{d \zeta}(\zeta)=(\chi-|\beta|)^{2}$;

(d) $\frac{d^{2} \rho}{d \zeta^{2}}(\zeta)=\chi \frac{\alpha^{2}}{\left(2 \alpha \zeta+4 \beta^{2} \zeta^{2}\right)^{3 / 2}}$ does not change sign.

Fig. 6 provides a graphical depiction of $\rho$ for $\chi>0, \alpha \geq 0$, and $\frac{\alpha}{2}-V_{\mathrm{s}, \mathrm{zero}}\left(\theta_{\mathrm{s}, 0}^{-}\right)>0$. Similar figures could be drawn for other cases. The next result shows that these qualitative features of the Poincaré return map lead to a large region of attraction for an exponentially stable fixed-point.

Theorem 4 (Non-local convergence in the HZD): Consider $\rho: \mathcal{D}_{\rho} \rightarrow \mathbb{R}$, and suppose that

1) $(\chi-|\beta|)^{2}<1$,

2) $\chi>0$,

3) and there exists $\zeta^{*} \in \mathcal{D}_{\rho}$ such that $\rho\left(\zeta^{*}\right)=\zeta^{*}$ and $\frac{d \rho}{d \zeta}\left(\zeta^{*}\right)>0$.

Then, the following statements are true:

(a) $\zeta^{*}$ is the unique fixed-point of $\rho$;

(b) the set

$$
\tilde{\mathcal{D}}_{\rho}=\left\{\zeta \in \mathcal{D}_{\rho} \mid \frac{d \rho}{d \zeta}(\zeta)>0\right\}
$$

is unbounded and connected; and

(c) $\zeta^{*}$ is locally exponentially stable and every solution of $\zeta(k+1)=\rho(\zeta(k))$ initialized in $\tilde{\mathcal{D}}_{\rho}$ converges monotonically to $\zeta^{*}$.

The proof is given in Appendix V. This result shows that once the motion of the robot has settled to the hybrid zero dynamics, the domain of attraction of the periodic orbit is quite large. The analysis in Theorem 4 has not accounted for 


\begin{tabular}{c|c|c|c}
\hline & Femur & Tibia & Torso \\
\hline \hline Length $(\mathrm{m})$ & 0.4 & 0.4 & 0.625 \\
\hline Mass $(\mathrm{kg})$ & 6.8 & 3.2 & 17.0 \\
\hline Inertia about COM $\left(\mathrm{kg}-\mathrm{m}^{2}\right)$ & 0.47 & 0.20 & 1.33 \\
\hline
\end{tabular}

\section{TABLE I}

EXPERIMENTALLY MEASURED PARAMETERS FOR RABBIT.

the peak torque of the actuators and the allowed friction cone at the support leg end. This theorem should thus be viewed as stating that such physical considerations will determine the limits on the region of attraction, and that the semi-global convergence of the control loop per se is not the key limiting factor.

For all of the examples worked by the authors, if an exponentially stable fixed point was found, hypotheses (1), (2) and (3) of Theorem 4 have always held as well. In particular, $\mu$ was always greater than 0.4 and $\tilde{\mathcal{D}}_{\rho}$ equalled $\mathcal{D}_{\rho}$, that is, the Poincaré map was always strictly increasing on the region of interest. In the case of Raibert's hopper, the Poincaré map was unimodal-and thus not strictly increasing on the domain of interest [17]. Nevertheless, semi-global stability was established using a more powerful analysis method due to Singer [53] and Guckenheimer [54].

\section{Stability in the full-order model}

Under the assumptions made in Section VI-A on the stancephase controller, Theorem 2 of [2] guarantees that asymptotically stable orbits in the hybrid zero dynamics (63) are also asymptotically stable in the full-order hybrid model (62) (for a discussion of exponential stability, see Remark 1 in [13]). By Theorem 1, the Poincaré return maps of (62) and (56) are identical. Hence, once asymptotic stability of an orbit has been proven in (62), it follows that the orbit is also asymptotically stable for (56). Putting all of this together, proving the existence and stability of an orbit in the hybrid zero dynamics of running leads to the desired conclusions in the state space of the closed-loop hybrid model (56).

\section{ILLUSTRATION ON RABBIT}

The analytical results of Section VII make it straightforward to determine if a control law of the kind specified in Section VI leads to the existence of a stable periodic orbit. However, proposing specific values for the output functions so that the evolution of the robot is energetically efficient, while respecting actuator limits, the friction cone at the contact point of the leg end, and lift-off at the beginning of the flight phase, is nearly impossible to do by intuition. Here, the feedback designs will be based on optimization.

Using the method proposed in [11], time-trajectories of (29), corresponding to average running speeds varying from $0.5 \mathrm{~m} / \mathrm{s}$ to $2.75 \mathrm{~m} / \mathrm{s}$ and parameter values given in Tab. I, were determined for RABBIT (see [1] for details on the planar, bipedal robot, RABBIT). The running trajectories satisfy $\ddot{\mathbf{y}}_{1}>0$ at the beginning of the flight phase, the duration of the flight phase

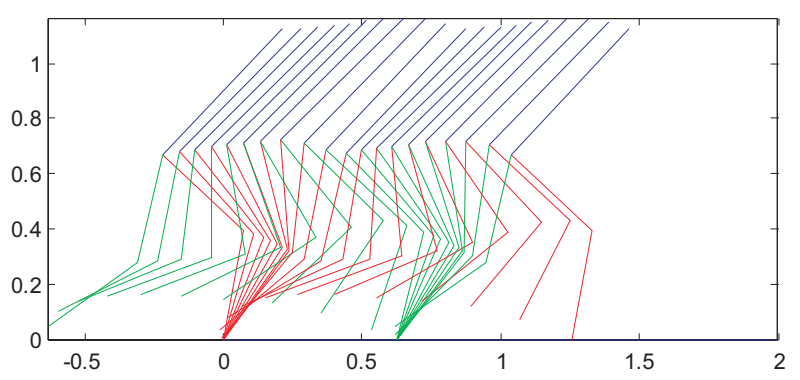

Fig. 7. Stick diagram for a running trajectory with average speed $1.5 \mathrm{~m} / \mathrm{s}$.

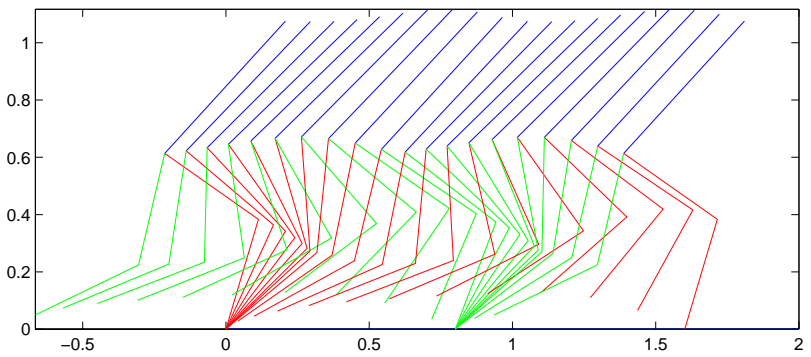

Fig. 8. Stick diagram for a running trajectory with average speed $2.5 \mathrm{~m} / \mathrm{s}$.

is at least $25 \%$ of the duration of a stride, and the required coefficient of friction is less than $2 / 3$. Stick-figure diagrams corresponding to the running motions of $1.5 \mathrm{~m} / \mathrm{s}$ and $2.5 \mathrm{~m} / \mathrm{s}$ are given in Figs. 7 and 8.

Denote by $\mathcal{O}$ the path traced out in the state spaces of the hybrid model of the robot by any one of these running trajectories. It was checked that $\mathcal{O}$ intersects $\mathcal{S}_{\mathrm{s}}^{\mathrm{f}}$ and $\mathcal{S}_{\mathrm{f}}^{\mathrm{s}}$ exactly once; define $x_{\mathrm{f}}^{*-}=\mathcal{O} \cap \mathcal{S}_{\mathrm{f}}^{\mathrm{s}}$ and $x_{\mathrm{s}}^{*-}=\mathcal{O} \cap \mathcal{S}_{\mathrm{s}}^{\mathrm{f}}$. The goal is to design a time-invariant state-feedback controller à la Section VI that has $\mathcal{O}$ as its asymptotically-stable periodic orbit. Recall that designing the controller is equivalent to specifying the output functions in (33) and (47) and the parameter update-law in (56).

\section{A. Stance Phase Controller Design}

On the basis of $x_{\mathrm{f}}^{*-}$ and $x_{\mathrm{s}}^{*-}$, the values of $q_{0}^{\mathrm{s}+}$ (the initial configuration in stance on the periodic orbit), $q_{0}^{\mathrm{s}-\mathrm{-}}$ (final configuration in stance on the periodic orbit), $\dot{q}_{0}^{\text {s+ }}$ (normalized initial velocity in stance on the periodic orbit; see (42)), and $\dot{q}_{0}^{\mathrm{s}-}$ (normalized final velocity in stance on the periodic orbit ${ }^{7}$ ) are easily deduced, which in turn give the initial and final values of $\theta_{\mathrm{s}}$ on the periodic orbit, $\theta_{\mathrm{s}, 0}^{+}$and $\theta_{\mathrm{s}, 0}^{-}$.

As in [5], an output $y_{\mathrm{s}}=h_{\mathrm{s}}(q):=q_{\mathrm{b}}-h_{d, \mathrm{~s}} \circ \theta_{\mathrm{s}}(q)$ is designed so that it satisfies the boundary condition and vanishes (nearly) along the stance phase of the periodic orbit, and thus the orbit will be an integral curve of the stance-phase zero dynamics. For this, the function $h_{d, \mathrm{~s}}$ was selected to be a fourth-order polynomial in $\theta_{\mathrm{s}}$. The design method in [11] that is used to compute the periodic orbit essentially guarantees that the technical conditions of Section VI are satisfied for $h_{\mathrm{s}}$; nevertheless, the conditions were formally verified. Once $h_{\mathrm{s}}$ is known, so is $\mathcal{Z}_{\mathrm{s}}$, and, by construction, $\mathcal{O} \cap T \mathcal{Q}_{\mathrm{s}} \subset \mathcal{Z}_{\mathrm{s}}$.

${ }^{7}$ In (42), replace evaluation at $q_{0}^{\mathrm{s}-}$ with $q_{0}^{\mathrm{s}+}$. 


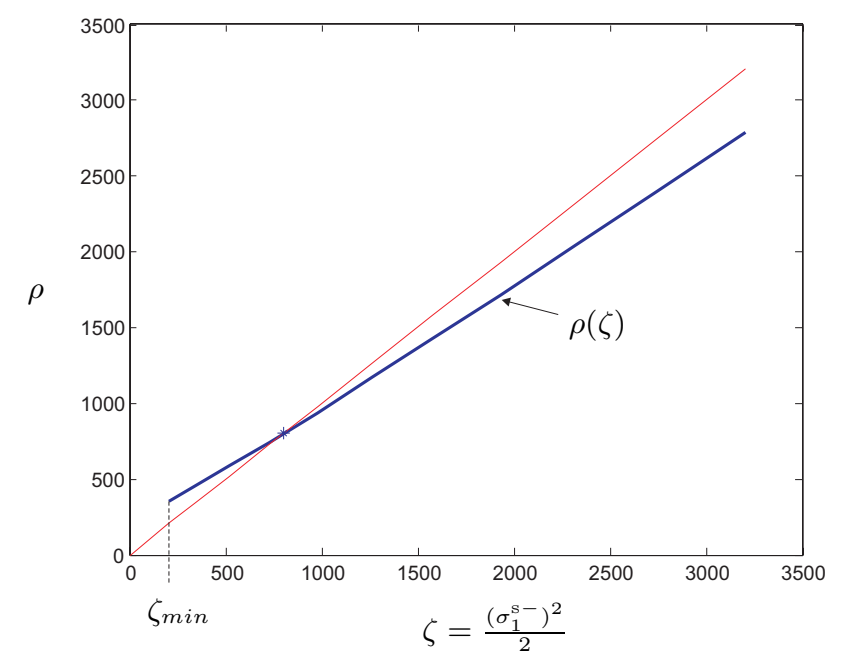

Fig. 9. Running at $1.5 \mathrm{~m} / \mathrm{s}$. The restricted Poincare map (bold) associated with the closed-loop system. The fixed point occurs where the graph of $\rho$ intersects the graph of the identity map (thin line).

\section{B. Stability of the periodic orbits}

The data required to determine the restricted Poincaré map $\rho$ in Theorem 3 and Theorem 4 can be computed directly from $h_{d, \mathrm{~s}}$. This was carried out for each of the running trajectories studied in this paper. The numerical values are summarized in Tab. II. In each case, $\mu<1$ and hence if a flightphase controller can be determined to meet the conditions of Theorem 3, the corresponding orbit will be asymptotically stable. Note that slower running speeds yield smaller values of $\mu$. So, for fast running, the convergence toward the periodic orbit will be slow. A plot of the restricted Poincaré map is provided in Fig. 9 for the trajectory corresponding to an average speed of $1.5 \mathrm{~m} / \mathrm{s}$.

\section{Flight Phase Controller Design}

The flight phase controller, $y_{\mathrm{f}}=h_{\mathrm{f}}\left(q_{\mathrm{f}}, a_{\mathrm{f}}\right):=q_{\mathrm{b}}-$ $h_{d, \mathrm{f}}\left(\mathbf{x}_{\mathrm{cm}}, a_{\mathrm{f}}\right), a_{\mathrm{f}}=w_{\mathrm{s}}^{\mathrm{f}}\left(x_{\mathrm{s}}^{-}\right)$, is to be designed so that trajectories of the closed-loop system that takeoff from the stancephase zero dynamics manifold, $\mathcal{Z}_{\mathrm{s}}$, land on $\mathcal{Z}_{\mathrm{s}}$; moreover, the landing configuration should be independent of the robot's takeoff velocity from $\mathcal{Z}_{\mathrm{s}}$. Since from Section VIII-A the initial stance-phase configuration of the robot on the periodic orbit is equal to $q_{0}^{\mathrm{s}+}$, these two conditions become

$$
\begin{aligned}
\Delta\left(\tilde{\mathcal{S}} \cap \mathcal{Z}_{\mathrm{s}}\right) & \subset \quad \mathcal{Z}_{\mathrm{s}} \\
\pi \circ \Delta\left(\tilde{\mathcal{S}} \cap \mathcal{Z}_{\mathrm{s}}\right) & =q_{0}^{\mathrm{s}+},
\end{aligned}
$$

where, as before, $\pi: T \mathcal{Q}_{\mathrm{s}} \rightarrow \mathcal{Q}_{\mathrm{s}}$ is the canonical projection. The design of the controller can now be broken down into several steps. First, (77) and (78) will be translated from boundary conditions on configuration and velocity at the beginning of the (next) stance phase, into boundary conditions at the end of the (current) flight phase. This will result in control objectives for the configuration and velocity of the body coordinates and for the overall orientation of the robot at landing. In a second step, because the body coordinates $q_{\mathrm{b}}$ are directly actuated, it is straightforward to design a family of functions $h_{d, \mathrm{f}}\left(\mathbf{x}_{\mathrm{cm}}, a_{\mathrm{f}}\right)$ that achieve the boundary conditions on the body-coordinate configuration and velocity, once the flight duration is determined from the ballistic motion of the robot's center of mass. The final step is more difficult because it is indirect: adjust the evolution of the body coordinates as a function of the takeoff velocity so as to achieve a desired orientation $q_{5}$ of the robot at landing.

To begin the first step, observe that because $\left(q_{0}^{\mathrm{s}+}, \dot{q}\right)$ is in $\pi^{-1}\left(q_{0}^{\mathrm{s}+}\right) \cap \mathcal{Z}_{\mathrm{s}}$ if, and only if, $\dot{q}=\dot{q}_{0}^{\mathrm{s}+} \sigma_{1}^{\mathrm{s}+}$ for some $\sigma_{1}^{\mathrm{s}+} \in \mathbb{R}$, and $\left(q_{0}^{\mathrm{s}-}, \dot{q}\right)$ is in $\tilde{\mathcal{S}} \cap \mathcal{Z}_{\mathrm{s}}$ if, and only if, $\dot{q}=\dot{q}_{0}^{\mathrm{s}-} \sigma_{1}^{\mathrm{s}-}$ for some $\sigma_{1}^{\mathrm{s}-} \in \mathbb{R}$, conditions (77) and (78) are equivalent to

$$
\forall \sigma_{1}^{\mathrm{s}-}, \exists \sigma_{1}^{\mathrm{s}+} \text { s.t. } \Delta\left(q_{0}^{\mathrm{s}-}, \dot{q}_{0}^{\mathrm{s}-} \sigma_{1}^{\mathrm{s}-}\right)=\left(q_{0}^{\mathrm{s}+}, \dot{q}_{0}^{\mathrm{s}+} \sigma_{1}^{\mathrm{s}+}\right) .
$$

¿From Theorem 2, it follows that $\sigma_{1}^{\mathrm{s}+}=\delta\left(\sigma_{1}^{\mathrm{s}-}\right)$, and hence (79) is equivalent to

$$
\Delta\left(q_{0}^{\mathrm{s}-}, \dot{q}_{0}^{\mathrm{s}-} \sigma_{1}^{\mathrm{s}-}\right)=\left(q_{0}^{\mathrm{s}+}, \dot{q}_{0}^{\mathrm{s}+} \delta\left(\sigma_{1}^{\mathrm{s}-}\right)\right),
$$

which gives specific boundary conditions, just after impact, to be met by the design of the flight phase controller. In particular, recalling that $q=\left(q_{b}^{\prime}, q_{5}\right)^{\prime}$, it is seen that (80) places constraints on the body configuration variables and their derivatives, and on the overall orientation of the robot, $q_{5}$, while the constraint on $\dot{q}_{5}$ is equivalent to $\sigma_{1}^{\mathrm{s}+}=\delta\left(\sigma_{1}^{\mathrm{s}-}\right)$, if the other constraints are met.

For the purpose of computation, it is convenient to transform (80) to conditions in the flight-phase state space, $T \mathcal{Q}_{\mathrm{f}}$, instead of the stance-phase state space, $T \mathcal{Q}_{\mathrm{s}}$. This is done as follows: the boundary conditions (80) specify the height of the center of mass at impact, and from this information, the flight time, $t_{\mathrm{f}}$, is computed for any initial condition in $\tilde{\mathcal{S}} \cap \mathcal{Z}_{\mathrm{s}}$; see (100) in the Appendix. Using (101) and (46), the velocity of the center of mass can be expressed as a function of $\sigma_{1}^{\mathrm{s}-}$,

$$
\left[\begin{array}{c}
\dot{\mathbf{x}}_{\mathrm{cm}}^{f-} \\
\dot{\mathbf{y}}_{\mathrm{cm}}^{f-}
\end{array}\right]=\left[\begin{array}{c}
\lambda_{x}\left(q_{0}^{\mathrm{s}-}\right) \sigma_{1}^{\mathrm{s}-} \\
-\sqrt{\left(\lambda_{y}\left(q_{0}^{\mathrm{s}-}\right) \sigma_{1}^{\mathrm{s}-}\right)^{2}-2 g\left(\mathbf{y}_{\mathrm{cm}}^{\mathrm{s}+}-\mathbf{y}_{\mathrm{cm}}^{\mathrm{s}-}\right)}
\end{array}\right]_{(81)}
$$

The impact model (20), can be rewritten to define the angular velocity at the end of flight satisfying (80):

$$
\begin{gathered}
\dot{q}^{\mathrm{f}-}=A^{-1}\left(A+m \frac{\partial \mathbf{f}_{2}{ }^{\prime}}{\partial q} \frac{\partial \mathbf{f}_{2}}{\partial q}\right) R^{-1} \dot{q}_{0}^{\mathrm{s}+} \delta\left(\sigma_{1}^{\mathrm{s}-}\right)+ \\
+m A^{-1} \frac{\partial \mathbf{f}_{2}^{\prime}}{\partial q}\left[\begin{array}{c}
\dot{\mathbf{x}}_{\mathrm{cm}}^{f-} \\
\dot{\mathbf{y}}_{\mathrm{cm}}^{f-}
\end{array}\right] .
\end{gathered}
$$

These last two equations define a function $\dot{\bar{q}}_{0}\left(q_{0}^{\mathrm{s}+}, \sigma_{1}^{\mathrm{s}-}\right)$ such that $(80)$ is equivalent to

$$
\begin{aligned}
& q_{0}^{\mathrm{f}-}=R^{-1} q_{0}^{\mathrm{s}+} \\
& \dot{q}^{\mathrm{f}-}=\dot{\bar{q}}_{0}\left(q_{0}^{\mathrm{s}+}, \sigma_{1}^{\mathrm{s}-}\right) .
\end{aligned}
$$

In summary, the objective of the flight-phase controller is to meet the boundary conditions given in (83). Meeting these two conditions will assure that invariance of $\mathcal{Z}_{\mathrm{s}}$ under the composition of the flight phase and impact model is achieved, (77), and that configuration determinism at transition, (78), is also met; see Figs. 2 and 3. 


\begin{tabular}{c|cc|cccccc}
\hline $\begin{array}{c}\text { Average } \\
\text { velocity }\end{array}$ & $V_{\text {zero }}\left(\theta_{\mathrm{s}}^{-}\right)$ & $V_{\mathrm{s}, \text { zero }}^{\max }$ & $\begin{array}{c}\mathbf{x}_{\mathrm{cm}}^{\mathrm{s}+} \\
(\mathrm{cm})\end{array}$ & $\begin{array}{c}\mathbf{y}_{\mathrm{cm}}^{\mathrm{s}+} \\
(\mathrm{cm})\end{array}$ & $\begin{array}{c}\mathbf{x}_{\mathrm{cm}}^{\mathrm{s}-} \\
(\mathrm{cm})\end{array}$ & $\begin{array}{c}\mathbf{y}_{\mathrm{cm}}^{\mathrm{s}-} \\
(\mathrm{cm})\end{array}$ & $\begin{array}{c}\lambda_{x}\left(q_{0}^{\mathrm{s}-}\right) \\
\left(10^{-2}\right)\end{array}$ & $\begin{array}{c}\lambda_{y}\left(q_{0}^{\mathrm{s}-}\right) \\
\left(10^{-3}\right)\end{array}$ \\
\hline \hline $0.50 \mathrm{~m} / \mathrm{s}$ & -66 & 21 & -6.8 & 62.4 & 14.0 & 69.8 & 3.74 & 5.4 \\
$0.75 \mathrm{~m} / \mathrm{s}$ & -114 & 36 & -8.8 & 62.1 & 18.4 & 68.8 & 3.83 & 3.3 \\
$1.00 \mathrm{~m} / \mathrm{s}$ & -168 & 54 & -10.9 & 61.5 & 22.6 & 67.5 & 3.95 & 2.3 \\
$1.25 \mathrm{~m} / \mathrm{s}$ & -219 & 74 & -12.9 & 60.5 & 26.4 & 65.7 & 4.09 & 2.0 \\
$1.50 \mathrm{~m} / \mathrm{s}$ & -258 & 100 & -15.1 & 59.3 & 29.6 & 63.8 & 4.27 & 2.3 \\
$1.75 \mathrm{~m} / \mathrm{s}$ & -274 & 134 & -17.7 & 58.1 & 32.3 & 61.7 & 4.48 & 3.0 \\
$2.00 \mathrm{~m} / \mathrm{s}$ & -285 & 167 & -20.1 & 56.7 & 34.6 & 59.7 & 4.69 & 3.3 \\
$2.25 \mathrm{~m} / \mathrm{s}$ & -306 & 123 & -17.5 & 55.6 & 34.0 & 59.1 & 4.78 & 3.9 \\
$2.50 \mathrm{~m} / \mathrm{s}$ & -309 & 81 & -14.4 & 54.7 & 32.5 & 59.0 & 4.85 & 5.0 \\
$2.75 \mathrm{~m} / \mathrm{s}$ & -260 & 70 & -13.2 & 55.2 & 29.8 & 58.6 & 4.91 & 5.0 \\
\hline
\end{tabular}

\begin{tabular}{c|ccc|cc|cc}
\hline $\begin{array}{c}\text { Average } \\
\text { velocity }\end{array}$ & $\alpha$ & $\begin{array}{c}\beta \\
\left(10^{-2}\right)\end{array}$ & $\chi$ & $\zeta_{\min }$ & $\zeta^{*}$ & $\mu$ & $\chi-|\beta|$ \\
\hline \hline $0.50 \mathrm{~m} / \mathrm{s}$ & 9.12 & -1.37 & 0.926 & 53 & 151 & 0.695 & 0.832 \\
$0.75 \mathrm{~m} / \mathrm{s}$ & 14.26 & -1.07 & 0.926 & 88 & 275 & 0.708 & 0.838 \\
$1.00 \mathrm{~m} / \mathrm{s}$ & 19.04 & -0.92 & 0.931 & 125 & 434 & 0.729 & 0.850 \\
$1.25 \mathrm{~m} / \mathrm{s}$ & 23.34 & -0.96 & 0.940 & 164 & 615 & 0.754 & 0.866 \\
$1.50 \mathrm{~m} / \mathrm{s}$ & 27.33 & -1.29 & 0.955 & 206 & 801 & 0.785 & 0.887 \\
$1.75 \mathrm{~m} / \mathrm{s}$ & 30.84 & -1.99 & 0.976 & 253 & 982 & 0.826 & 0.914 \\
$2.00 \mathrm{~m} / \mathrm{s}$ & 32.77 & -2.47 & 0.990 & 294 & 1162 & 0.856 & 0.932 \\
$2.25 \mathrm{~m} / \mathrm{s}$ & 29.56 & -2.52 & 0.986 & 231 & 1327 & 0.859 & 0.922 \\
$2.50 \mathrm{~m} / \mathrm{s}$ & 23.69 & -2.66 & 0.984 & 161 & 1503 & 0.870 & 0.916 \\
$2.75 \mathrm{~m} / \mathrm{s}$ & 15.91 & -2.45 & 0.994 & 127 & 1729 & 0.908 & 0.940 \\
\hline
\end{tabular}

TABLE II

StABILITY ANALYSIS OF VARIOUS RUNNING MOTIONS. IF $\zeta>\zeta_{\min }, \operatorname{THEN} \zeta \in \mathcal{D}_{\rho}$.

The design of $h_{d, \mathrm{f}}$ can now be given in two more steps. First, define ${ }^{8}$

$$
\tau\left(\mathbf{x}_{\mathrm{cm}}, \sigma_{1}^{\mathrm{s}-}\right)=\frac{\mathbf{x}_{\mathrm{cm}}-\mathbf{x}_{\mathrm{cm}}^{\mathrm{f}+}}{t_{\mathrm{f}} \dot{\mathbf{x}}_{\mathrm{cm}}^{\mathrm{f}+}}=\frac{\mathbf{x}_{\mathrm{cm}}-\mathbf{x}_{\mathrm{cm}}^{\mathrm{f}+}}{t_{\mathrm{f}} \lambda_{x}\left(q_{0}^{\mathrm{s}-}\right) \sigma_{1}^{\mathrm{s}-}} ;
$$

the real-valued function $\tau$ varies between 0 and 1 and can be used to parameterize trajectories from $\tilde{\mathcal{S}} \cap \mathcal{Z}_{\mathrm{s}}$ to $\pi^{-1}\left(q_{0}^{\mathrm{s}+}\right) \cap \mathcal{Z}_{\mathrm{s}}$ in a neighborhood of the periodic orbit. Choose a function $\mathrm{fcn}\left(a_{1}, \cdots, a_{5}\right):[0,1] \rightarrow \mathbb{R}^{4}$ such that

$$
\begin{aligned}
\operatorname{fcn}\left(a_{1}, \cdots, a_{5}\right)(0) & =a_{1} \\
\frac{d \mathrm{fcn}}{d \tau}\left(a_{1}, \cdots, a_{5}\right)(0) & =a_{2} \\
\operatorname{fcn}\left(a_{1}, \cdots, a_{5}\right)(1) & =a_{3} \\
\frac{d \mathrm{fcn}}{d \tau}\left(a_{1}, \cdots, a_{5}\right)(1) & =a_{4},
\end{aligned}
$$

and there exist $a_{1}^{*}, \cdots, a_{5}^{*}$ for which $q_{b}-\mathrm{fcn}\left(a_{1}^{*}, \cdots, a_{5}^{*}\right)(\tau)$ (nearly) vanishes on $\mathcal{O}$. Here, this was accomplished with a fourth order polynomial. Off of the orbit, use (85) to solve for $a_{1}, \cdots, a_{4}$ as functions of $\sigma_{1}^{\mathrm{s}-}$ so that $q_{b}(\tau)=$ $\operatorname{fcn}\left(a_{1}, \cdots, a_{5}\right)(\tau)$ satisfies the constraints on the body coordinates imposed by (83). Specifically, set $a_{1}=\left(q_{0}^{\mathrm{s}-}\right)_{b}$, $a_{3}=\left(R^{-1} q_{0}^{\mathrm{s}+}\right)_{b}, a_{2}=\left(\dot{q}_{0}^{\mathrm{s}-} \sigma_{1}^{\mathrm{s}-}\right)_{b}$, and $a_{4}=\left(\dot{\bar{q}}_{0}\left(q_{0}^{\mathrm{s}+}, \sigma_{1}^{\mathrm{s}-}\right)\right)_{b}$. Define

$$
h_{d, \mathrm{f}}\left(\mathbf{x}_{\mathrm{cm}}, \sigma_{1}^{\mathrm{s}-}, a_{5}\right):=\mathrm{fcn}\left(a_{1}, \cdots, a_{5}\right)(\tau)
$$

with $a_{i}\left(\sigma_{1}^{\mathrm{s}-}\right), i=1, \ldots, 4$ and $\tau\left(\mathbf{x}_{\mathrm{cm}}, \sigma_{1}^{\mathrm{s}-}\right)$ as determined above. Define $q_{5}(0)=\left(q_{0}^{\mathrm{s}-}\right)_{5}$ and $q_{5, d}=\left(R^{-1} q_{0}^{\mathrm{s}+}\right)_{5}$.

In the final step, the goal is to select $a_{5}$ as a function of $\sigma_{1}^{\mathrm{s}-}$ so that the $q_{5}$-component-the overall orientation of

\footnotetext{
${ }^{8}$ Note that $\mathbf{x}_{\mathrm{cm}}^{\mathrm{f}+}=\mathbf{x}_{\mathrm{cm}}^{\mathrm{s}-}$.
}

the robot-satisfies the landing constraint. This is done as follows. The output (86) satisfies all of the conditions of Section VI, and hence the evolution of $q_{5}$ in the flight-phase zero dynamics is given by $\dot{q}_{5}=\kappa_{1, \mathrm{f}}\left(\sigma_{\mathrm{cm}}, \mathbf{x}_{\mathrm{cm}}, \dot{\mathbf{x}}_{\mathrm{cm}}, \sigma_{1}^{\mathrm{s}-}, a_{5}\right)$. In the flight phase, $\sigma_{\mathrm{cm}}$ and $\dot{\mathbf{x}}_{\mathrm{cm}}$ are constant and can be substituted by their values from $\tilde{\mathcal{S}} \cap \mathcal{Z}_{\mathrm{s}}$. In addition, $\mathbf{x}_{\mathrm{cm}}(t)=$ $\mathbf{x}_{\mathrm{cm}}^{\mathrm{s}-}+t \lambda_{x}\left(q_{0}^{\mathrm{s}-}\right) \sigma_{1}^{\mathrm{s}-}$. Hence, $\dot{q}_{5}=\tilde{\kappa}_{1, \mathrm{f}}\left(t, \sigma_{1}^{\mathrm{s}-}, a_{5}\right)$. Letting $\sigma_{1}^{* \mathrm{~S}-}$ denote the value of $\sigma_{1}^{\mathrm{s}-}$ on the orbit, $\mathcal{O}, q_{5, d}=q_{5}(0)+$ $\int_{0}^{t_{f}} \tilde{\kappa}_{1, \mathrm{f}}\left(t, \sigma_{1}^{* \mathrm{~s}-}, a_{5}^{*}\right) d t$ is satisfied because, by construction of the output, the orbit corresponds to an integral curve of the flight-phase zero dynamics. Finally, it is verified (numerically) that

$$
\left.\frac{\partial}{\partial a_{5}}\left(q_{5, d}-q_{5}(0)-\int_{0}^{t_{f}} \tilde{\kappa}_{1, \mathrm{f}}\left(t, \sigma_{1}^{* \mathrm{~s}-}, a_{5}\right) d t\right)\right|_{a_{5}=a_{5} *} \neq 0
$$

and thus by the implicit function theorem, there exists an open subset about $\sigma_{1}^{* \mathrm{~s}-}$ and a differentiable function $\tilde{w}_{\mathrm{s}}^{\mathrm{f}}$ such that $\tilde{w}_{\mathrm{s}}^{\mathrm{f}}\left(\sigma_{1}^{* \mathrm{~s}-}\right)=a_{5}^{*}$ and

$$
q_{5, d}=q_{5}(0)+\int_{0}^{t_{f}} \tilde{\kappa}_{1, \mathrm{f}}\left(t, \sigma_{1}^{\mathrm{s}-}, \tilde{w}_{\mathrm{s}}^{\mathrm{f}}\left(\sigma_{1}^{\mathrm{s}-}\right)\right) d t .
$$

Since (88) is scalar while $a_{5}$ has four components, there exist an infinite number of solutions for $\tilde{w}_{\mathrm{s}}^{\mathrm{f}}$. Hence, a numerical optimization was performed to find, for each point in a neighborhood of $\sigma_{1}^{* \mathrm{~s}-}$, a value of $a_{5}$ that steers $q_{5}$ to $q_{5, d}$, while minimizing ${ }^{9}\left\|a_{5}-a_{5}^{*}\right\|$. The flight-phase control design is completed by formally defining $h_{d, \mathrm{f}}\left(q_{\mathrm{f}}, a_{\mathrm{f}}\right), a_{\mathrm{f}}:=\left(\sigma_{1}^{\mathrm{s}-}, a_{5}^{\prime}\right)^{\prime}$, and $w_{\mathrm{s}}^{\mathrm{f}}\left(x_{\mathrm{s}}^{-}\right):=\left(\sigma_{1}^{\mathrm{s}-}, \tilde{w}_{\mathrm{s}}^{\mathrm{f}}\left(\sigma_{1}^{\mathrm{s}-}\right)^{\prime}\right)^{\prime}$.

\footnotetext{
${ }^{9}$ Other criteria could be used, such as minimization of the torques in the flight phase. This latter criterion requires the computation of the torques via the dynamic model, and hence is costly in calculation time.
} 

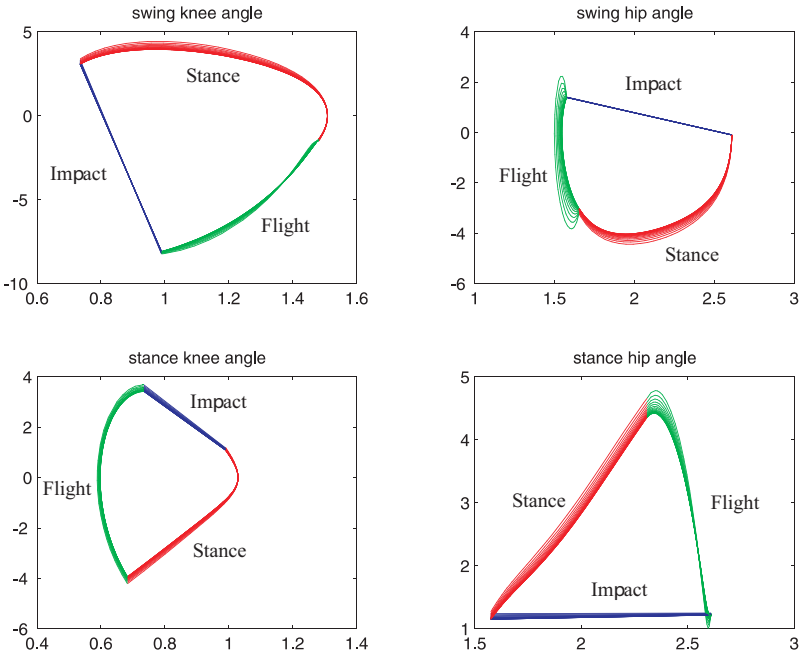

Fig. 10. Running at $1.5 \mathrm{~m} / \mathrm{s}$. The four graphs depict the relative joint angles in radians ( $\mathrm{x}$-axis) versus their velocities in radians per second (y-axis) in the stance, flight and impact phases: the swing knee angle (knee of leg-2), the swing hip angle (hip of leg-2), the stance knee angle (knee of leg-1) and the stance hip angle (hip of leg-1). At impact, the roles of the limbs are exchanged; as a consequence the configuration angles change at impact; see (30). Notice that the robot has the same configuration at each transition between phases. The plots indicate that a limit cycle is achieved.

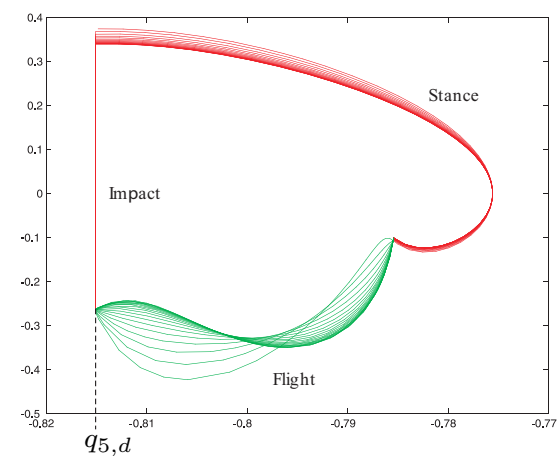

Fig. 11. Running at $1.5 \mathrm{~m} / \mathrm{s}$. The graph depicts torso angle in radians (xaxis) versus its velocity in radians per second (y-axis) in the stance and flight phases. Notice that the flight-phase controller has regulated the torso angle to its desired value of $q_{5, d}$ at impact. The plot indicates that a limit cycle is achieved.

\section{Simulation without modeling error}

The control law developed above has been simulated on a model of RABBIT for the various running motions. Assuming no modeling error and initializing the closed-loop system off of the periodic orbit—with the initial velocity $10 \%$ higher than the value on the periodic orbit-the simulation data presented in Figs. 10 to 17 are obtained for the running motions of $1.5 \mathrm{~m} / \mathrm{s}$ and $2.5 \mathrm{~m} / \mathrm{s}$.

For a running speed of $1.5 \mathrm{~m} / \mathrm{s}$ (resp., $2.5 \mathrm{~m} / \mathrm{s}$ ) Figs. 10 and 11 (resp., Figs. 14 and 15) show the phase-plane evolution of the configuration variables. The convergence to the periodic orbit is clear. By the design of the controller, the stance-phase evolution of the configuration variables does not change strideto-stride; only the velocities change. In the flight phase, (most notably, for the hips and the torso when running at $1.5 \mathrm{~m} / \mathrm{s}$ ), the path traced out is modified so that the robot lands in the desired state.
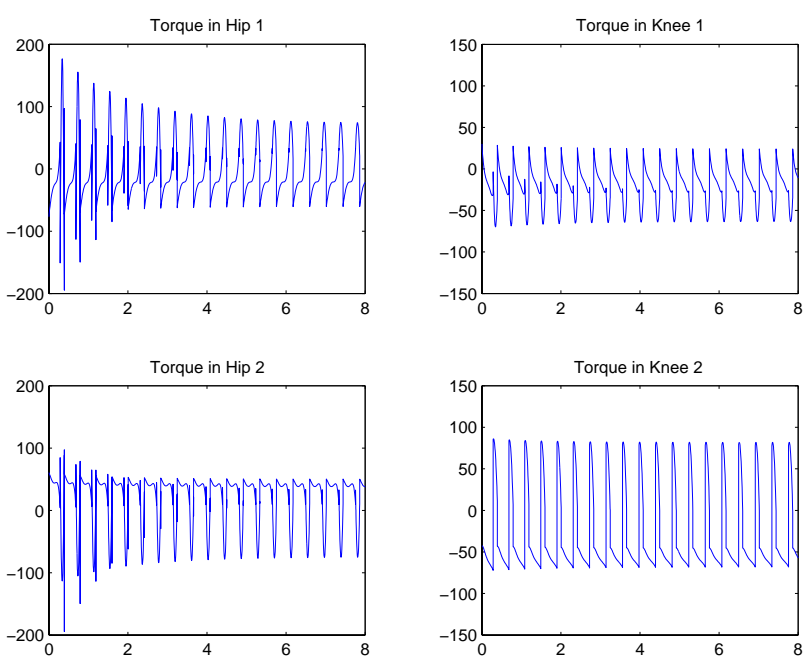

Fig. 12. Running at $1.5 \mathrm{~m} / \mathrm{s}$. The four graphs depict the joint torques in Newton-meters (y-axis) versus time in seconds (x-axis) in the stance and flight phases. Upon convergence to the periodic orbit, the achieved torques are very close to their optimal values. The torque is higher in the flight phase away from the periodic orbit, especially in the hips.
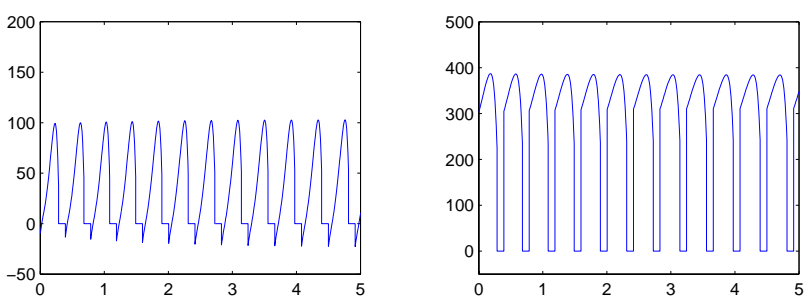

Fig. 13. Running at $1.5 \mathrm{~m} / \mathrm{s}$. The left graph depicts leg-1 (stance leg) horizontal force in Newtons (y-axis) versus time in seconds (x-axis) in the stance and flight phases. The right graph depicts vertical force (y-axis) versus time (x-axis) in the stance and flight phases. The impulsive forces existing during impact are not presented.
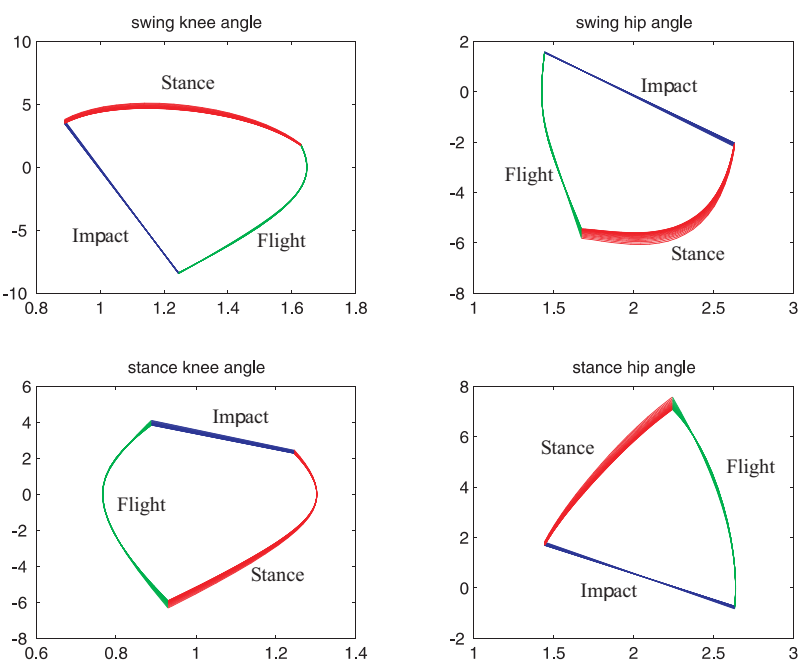

Fig. 14. Running at $2.5 \mathrm{~m} / \mathrm{s}$. The four graphs depict the relative joint angles in radians (x-axis) versus their velocities in radians per second (y-axis) in the stance, flight and impact phases: the swing knee angle (knee of leg-2), the swing hip angle (hip of leg-2), the stance knee angle (knee of leg-1) and the stance hip angle (hip of leg-1). At impact, the roles of the limbs are exchanged; as a consequence the configuration angles change at impact; see (30). Notice that the robot has the same configuration at each transition between phases. The plots indicate that a limit cycle is achieved. 


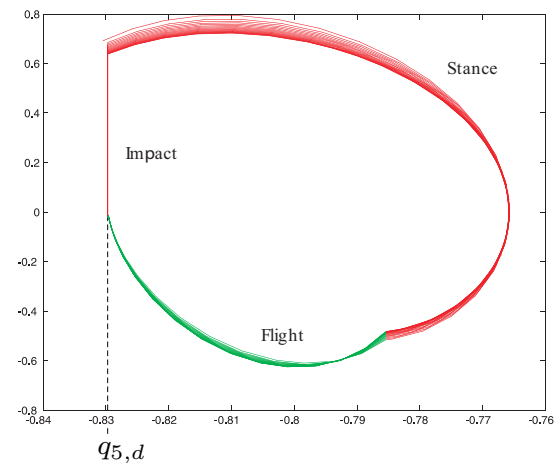

Fig. 15. Running at $2.5 \mathrm{~m} / \mathrm{s}$. The graph depicts torso angle in radians (xaxis) versus its velocity in radians per second (y-axis) in the stance and flight phases. Notice that the flight-phase controller has regulated the torso angle to its desired value of $q_{5, d}$ at impact. The plot indicates that a limit cycle is achieved.
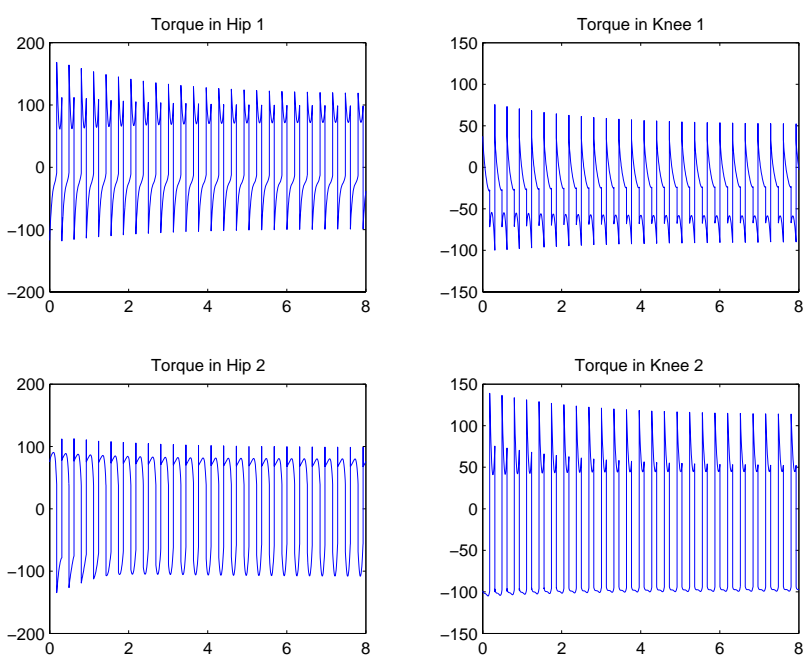

Fig. 16. Running at $2.5 \mathrm{~m} / \mathrm{s}$. The four graphs depict the joint torques in Newton-meters (y-axis) versus time in seconds (x-axis) in the stance and flight phases. Upon convergence to the periodic orbit, the achieved torques are very close to their optimal values. The torque is higher in the flight phase away from the periodic orbit, especially in the hips.
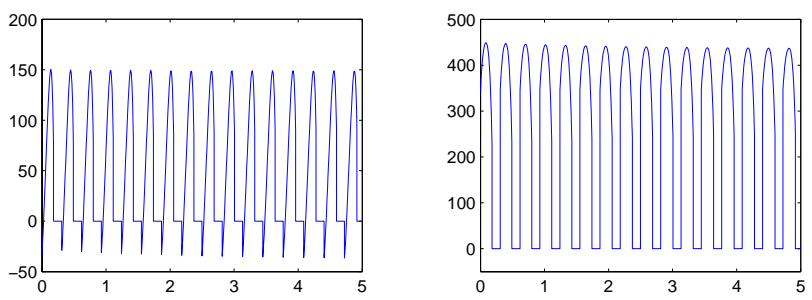

Fig. 17. Running at $2.5 \mathrm{~m} / \mathrm{s}$. The left graph depicts leg-1 (stance leg) horizontal force in Newtons (y-axis) versus time in seconds (x-axis) in the stance and flight phases. The right graph depicts vertical force (y-axis) versus time (x-axis) in the stance and flight phases. The impulsive forces existing during impact are not presented.
Figs. 12 and 16 depict the torques for running at $1.5 \mathrm{~m} / \mathrm{s}$ and $2.5 \mathrm{~m} / \mathrm{s}$, respectively. As the motion converges to the periodic orbit, the torques correspond to their optimal values, and hence are within the capabilities of the actuators. Off of the periodic orbit, the torques are significantly higher in the flight phase. For the slower $1.5 \mathrm{~m} / \mathrm{s}$-orbit, the torque increase occurs principally in the hips. For the faster $2.5 \mathrm{~m} / \mathrm{s}$-orbit, the torque increase is more evenly divided among the four actuators and is smaller in magnitude; the corresponding modification to the path in the flight phase is also smaller; see Figs. 14 and 15.

The reaction forces on leg-1 are provided in Figs. 13 and 17. These graphs show the alternating phases of single support and flight. The robot will not slip for a coefficient of friction greater than 0.5. The vertical force during the single support phase is very close to the weight of the robot (from Tab. I, its mass is $37 \mathrm{~kg}$ ).

\section{A Partial Robustness Evaluation}

The purpose of this section is to show that the proposed control strategy may still yield an attractive limit cycle even if the hypotheses made in the modeling of the robot, the control law's construction, and the analysis and simulation of the closed-loop system are not met exactly. The model of Section III assumed a rigid contact between the leg end and the ground. Here, a compliant contact model will be used [5]. This has several consequences. First of all, the seven DOF model of Section III-B will be used in the stance phase, with the position of the leg end with respect to the ground evolving freely as a function of the reaction forces provided by the compliant contact model. Secondly, the robot will enter the flight phase when the reaction forces at the leg end go to zero. Finally, the impact forces at touch down will be computed by the compliant model as well. In addition to these changes, parameter error will be introduced in the robot model.

\section{A. Compliant contact model}

In the experimental platform of RABBIT [1], the contact between the ends of the robot's legs and the ground is compliant and the ends of the legs may slip. A model that more closely reflects these points is summarized here. A more detailed discussion is available in [5] and the references therein.

The dynamic model consists of the full 7-DOF model of the biped (6) with the computation of the forces acting on the leg end being given by

$$
\begin{aligned}
F_{n} & =-\lambda^{a}|\mathbf{z}|^{n} \dot{\mathbf{z}}-\lambda^{b}|\mathbf{z}|^{n} \operatorname{sgn}(\dot{\mathbf{z}}) \sqrt{|\dot{\mathbf{z}}|}+k|\mathbf{z}|^{n} \\
F_{t} & =\left(\vartheta^{a} d+\vartheta^{b} \dot{d}+\vartheta^{c} \mathbf{v}+\vartheta^{d} \operatorname{sgn}(\dot{\mathbf{v}}) \sqrt{|\mathbf{v}|}\right)\left|F_{n}\right| \\
\dot{d} & =\mathbf{v}-|\mathbf{v}| \frac{\vartheta^{a}}{\vartheta^{e}} d,
\end{aligned}
$$

where $\mathbf{z} \leq 0$ is the penetration depth (if $\mathbf{z} \leq 0$, the leg is in contact with the ground, if $\mathbf{z}>0$, the leg is not in contact with the ground and the contact forces equal zero) and $\mathbf{v}$ is the relative velocity of the end of the leg with respect to the ground. This model supposes that the interface between the two contacting surfaces is a contact between bristles; the 


\begin{tabular}{c|c}
\hline Parameter & Value \\
\hline \hline$\lambda^{a}$ & $9 \times 10^{6}$ \\
\hline$\lambda^{b}$ & 0.3 \\
\hline$\vartheta^{c}$ & 0.18 \\
\hline$\vartheta^{d}$ & 0.3 \\
\hline
\end{tabular}

\begin{tabular}{c|c}
\hline Parameter & Value \\
\hline \hline$\vartheta^{a}$ & 260 \\
\hline$\vartheta^{b}$ & 0.6 \\
\hline$n$ & 1.5 \\
\hline$k$ & $25 \times 10^{5}$ \\
\hline$\vartheta^{e}$ & 0.285 \\
\hline
\end{tabular}

TABLE III

COMPLIANT CONTACT MODEL PARAMETERS

average deflection $d$ of the bristles is an internal state used to model dynamic friction. The numerical values used in the simulation, given in Tab. III, were adjusted for a nominal penetration of approximately $3 \mathrm{~mm}$ and to avoid rebound of the leg during the stance phase.

Together, the models (6) and (89) describe the robot's evolution in all phases of motion: flight, stance and impact. The robot's dynamics are then described by ordinary (nonhybrid) differential equations over the entire stride, even during the impact, which will now have a non-zero duration. With this model, contact forces at the leg end are continuous, which means in particular that they will not experience an instantaneous jump to zero at the transition from stance to flight as supposed in the development of the control law.

\section{B. Simulation with modeling error}

In addition to the structural change in the contact model, parametric modeling error is included. A deviation of $\pm 20 \%$ in the masses and inertias was introduced between the robot's design model and the simulation model; symmetry of the two legs was preserved. It is important to note that one consequence of parametric error is that there will be an error in the state of the robot at landing: because the flight-phase controller does not correspond to the simulation model, it will not correctly account of the conservation of angular momentum. Finally, saturation was introduced on the torques $( \pm 150 \mathrm{Nm})$ to take into account the limitations of the actuators of RABBIT.

Despite all of the differences between the simulation model and the model used to design the controller, the feedback controller illustrated in Section VIII is able to induce a stable running motion. This is shown in Figs. 18 to 21 for a nominal speed of $1.5 \mathrm{~m} / \mathrm{s}$. In the simulations, the controller was switched from the stance phase to flight phase when $\theta_{\mathrm{s}}(q)$ attained $\theta_{\mathrm{s}}^{-}$, and it was switched from flight phase to stance phase when the penetration of the leg end into the compliant surface exceeded $2 \mathrm{~mm}$. Due to the differences in the design and simulation models, the limit cycle does not correspond exactly to the theoretical prediction. The value of $\zeta^{*}$ calculated from the simulation data and the model parameters is 829 , whereas the value predicted with the rigid model and perfectly known parameters was 801 (see Tab. II). The average running speed was calculated to be $1.54 \mathrm{~m} / \mathrm{s}$, compared to the previous value of $1.50 \mathrm{~m} / \mathrm{s}$.

Figs. 18 and 19 show the evolution of the configuration variables in the phase plane; the convergence to a limit cycle
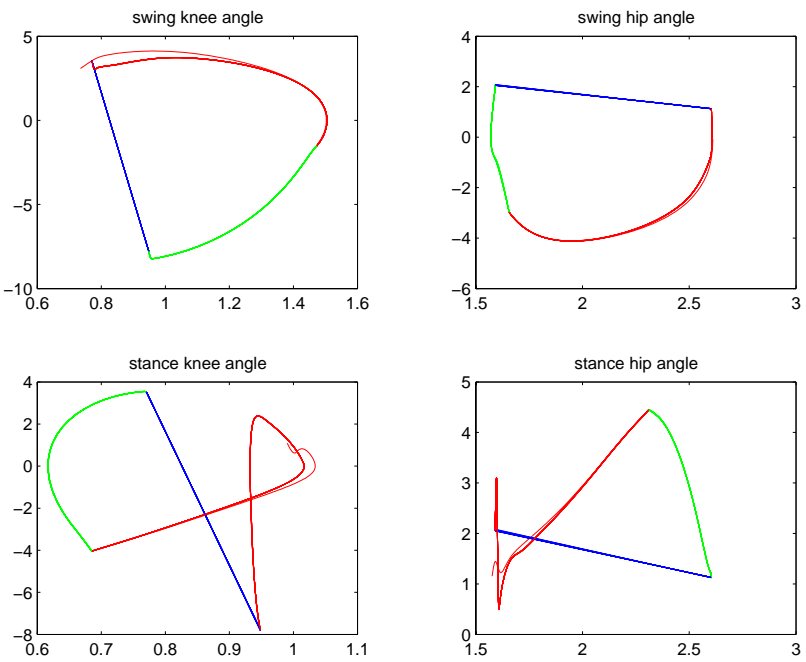

Fig. 18. Running at $1.5 \mathrm{~m} / \mathrm{s}$ with the compliant contact model and parametric modeling error. The four graphs depict the relative joint angles in radians ( $\mathrm{x}-$ axis) versus their velocities in radians per second (y-axis) in the stance, flight and impact phases: the swing knee angle (knee of leg-2), the swing hip angle (hip of leg-2), the stance knee angle (knee of leg-1) and the stance hip angle (hip of leg-1). At impact, the roles of the limbs are exchanged. Notice the abrupt change in the velocities at impact, especially in the stance leg. The plots indicate that a limit cycle is achieved.

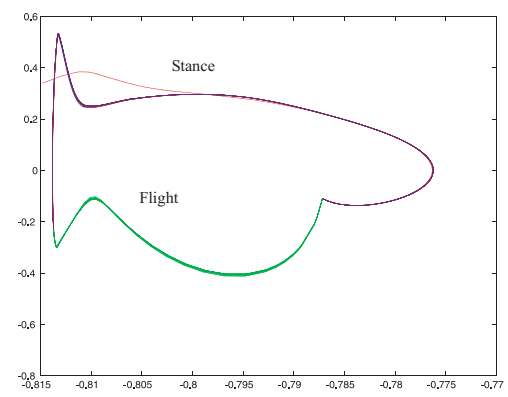

Fig. 19. Running at $1.5 \mathrm{~m} / \mathrm{s}$ with the compliant contact model and parametric modeling error. The graph depicts torso angle in radians (x-axis) versus its velocity in radians per second (y-axis) in the stance and flight phases. Notice that the flight-phase controller has approximately regulated the torso angle to its desired value of $q_{5, d}$ at impact. The plot indicates that a limit cycle is achieved.

is clear. At touchdown, the roles of the legs are swapped, as when the rigid contact model was used. At the beginning of the stance phase, the impact causes an abrupt change in the robot's velocities. At the moment of contact, the robot's velocities still correspond to their values from the flight phase. The control law sees this as a large set-point error and consequently applies a large torque, resulting in saturation; see Fig. 20. Once past the impact, the evolution of the relative angles is quite close to what was predicted with the rigid impact model; see Fig. 10 and Fig. 11. The perturbations during the flight phase are small because the initial condition of the simulation lies on the periodic orbit corresponding to the rigid contact model and no parametric modeling error.

The reaction forces on leg-1 are provided in Fig. 21. These graphs show the alternating phases of single support and flight. Except during impact, which is no longer instantaneous, the forces are close to the values predicted by the earlier 

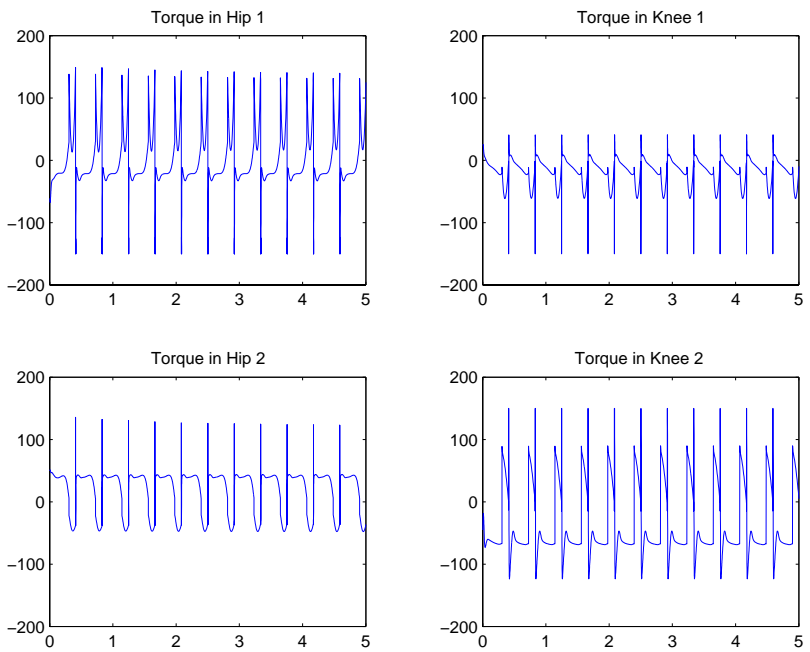

Fig. 20. Running at $1.5 \mathrm{~m} / \mathrm{s}$ with the compliant contact model and parametric modeling error. The four graphs depict the joint torques in Newton-meters (y-axis) versus time in seconds (x-axis) in the stance and flight phases. The torques are limited to $\pm 150 \mathrm{Nm}$. Upon convergence to the periodic orbit, the achieved torques are close to their optimal values. Prior to convergence, note the larger torques in the beginning of the stance phase due to a combination of modeling error and landing in the wrong state.
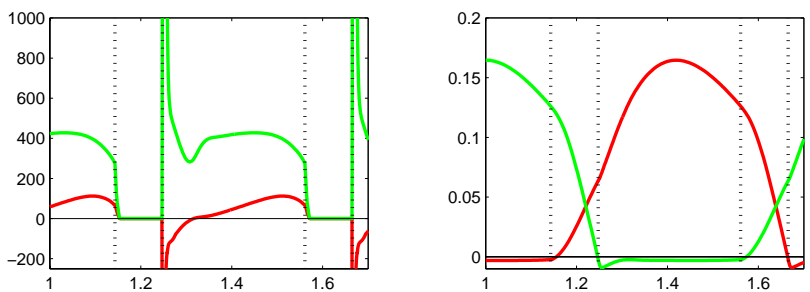

Fig. 21. Running at $1.5 \mathrm{~m} / \mathrm{s}$ with the compliant contact model and parametric modeling error. The left graph depicts leg-1 (stance leg) horizontal and vertical force components in Newtons (y-axis) versus time in seconds (x-axis) in the stance and flight phases. Large forces occur at touchdown; the maximal vertical force is close to $8000 \mathrm{~N}$ and the maximal horizontal force is close to $-4000 \mathrm{~N}$ with the compliant contact model. The vertical lines show the instant of transition between the control law phases. The right graph depicts vertical position of the leg end in meters ( $\mathrm{y}$-axis) versus time in seconds ( $\mathrm{x}$ axis) in the stance and flight phases. Notice that the flight control law induces the stance leg to lift off quickly and the reaction forces to go to zero.

simulation; see Fig. 13. The penetration of the stance leg end stabilizes at approximately $3 \mathrm{~mm}$. These two plots show clearly the very rapid liftoff of the stance leg to initiate the flight phase. Consequently, for the purposes of modeling, feedback design, and analysis, it is as reasonable to suppose an instantaneous transition to the flight phase as it is to suppose an instantaneous impact.

\section{Additional Event-BASEd CONTRol for RunNing}

Each of the feedback designs illustrated in Section VIII resulted in a nominally exponentially stable running motion. Indeed, this has been the case for all of the periodic orbits computed by the authors using the techniques in [11]. From Tab. II, it is seen that the rate of convergence to the periodic orbit decreases as the average running speed increases (that is, $\mu$ becomes closer to 1.0). The aim of this section is to illustrate how an additional event-based-control action studied in [4], [8], [13] can be profitably used to increase the rate of convergence to the periodic orbit. It will also be shown that the additional feedback action can be used to reduce the magnitude of the torques that are used in the flight phase to attain the desired landing state.

Remark 6: In Section IX-B, it was seen that modeling error alters the average running speed. As in [8], event-based control could also be used to attenuate the effects of modeling error on average running speed. In addition, it could be used to stabilize a periodic orbit that was nominally unstable under the feedback designs proposed so far.

\section{A. Deciding what to control}

Based on [15], it is natural to conjecture that modification of the target landing configuration stride-to-stride can be used to ameliorate the rate of convergence to the orbit and the peak torques in the flight phase. In particular, the horizontal distance between the center of mass and the stance leg has a strong effect ${ }^{10}$ on $\mu$. This suggests modifying the landing configuration in the direction $[0,0,1,0,0]^{\prime}$. On the other hand, the action of modifying the flight trajectory to obtain the correct orientation of the torso at landing is what leads to the higher torques. This suggests modifying the landing configuration in the direction $[0,0,0,0,1]^{\prime}$.

\section{B. Implementing stride-to-stride updates of landing configu- ration}

Let $q_{0}^{\mathrm{f}-}$ denote the nominal landing configuration for one of the running motions of Section VIII; see (83). Set the desired landing configuration at the $k$ th stride to be

$$
q_{0, d}^{\mathrm{f}-}(k)=q_{0}^{\mathrm{f}-}+\left[0,0, w_{1}(k), 0, w_{2}(k)\right]^{\prime},
$$

where the real scalars $w_{1}(k)$ and $w_{2}(k)$ are to be updated at the end of each stance phase. Through the impact map (30), a change in the desired landing configuration needs to be accompanied by a corresponding change in the desired initial stance configuration. Both of these changes entail stride-tostride parameter updates to the stance and flight controllers of Section VI. As a result, the restricted Poincaré map is now a function of $w_{1}(k)$ and $w_{2}(k)$ and can be viewed as a discretetime control system

$$
\zeta(k+1)=\rho\left(\zeta(k), w_{1}(k), w_{2}(k)\right)
$$

with state space $\tilde{\mathcal{S}} \cap \mathcal{Z}_{\mathrm{s}}$ and inputs $\left(w_{1}, w_{2}\right)^{\prime} \in \mathbb{R}^{2}$; see [13] for details. Linearizing (91) about the nominal fixed-point $\zeta^{*}$ corresponding to $w_{1}=0$ and $w_{2}=0$ results in

$$
\delta \zeta(k+1)=\mu \delta \zeta(k)+b_{1} \delta w_{1}(k)+b_{2} \delta w_{2}(k) .
$$

The value of $\mu$ is determined from Corollary 1 ; the sensitivities $b_{1}$ and $b_{2}$ are more easily determined numerically through a simulation of the model.

Linear state variable feedback $\delta w_{1}(k)=k_{1} \delta \zeta(k)$, $\delta w_{2}(k)=k_{2} \delta \zeta(k)$ can then be used to tradeoff peak torques

\footnotetext{
${ }^{10}$ When the heights of the center of mass at the beginning and end of the stance phase are the same, $\mu=(\chi-|\beta|)$, which is a function only of the horizontal position of the center of mass with respect to the stance leg end; see (66).
} 


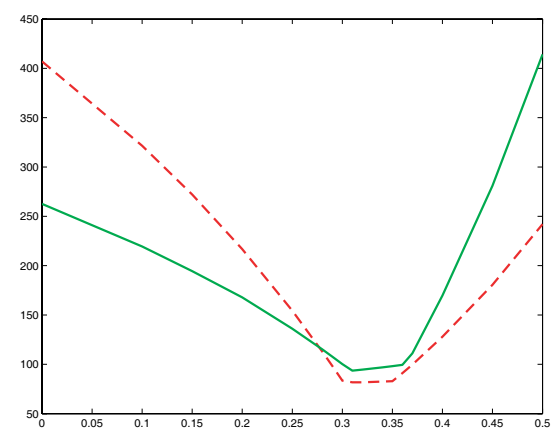

Fig. 22. A one-parameter search to minimize peak torque. Let $k_{2}=a k_{1}$. The graph depicts the maximal torque in Newton-meters (y-axis) versus the parameter $a$ (x-axis) for an initial velocity of the robot equal to $\pm 10 \%$ of its value on the periodic orbit (the solid line corresponds to $+10 \%$ and the dashed line corresponds to $-10 \%$ ). The best choice of parameter $a$ is $0.3<a<0.35$ to minimize the peak torque.

and the rate of convergence to the fixed point. For the running motion with average speed of $1.5 \mathrm{~m} / \mathrm{s}$, it was arbitrarily decided to place the closed-loop eigenvalue at $\mu_{d}=2 / 3$. A one-parameter search was then performed to minimize the torques in the flight phase when the velocity upon entering the flight phase differed from the value on the periodic orbit by $\pm 10 \%$, subject to $\mu+k_{1} b_{1}+k_{2} b_{2}=2 / 3$; see Fig. 22 . This resulted in $k_{1}=7.8 \times 10^{-5}$ and $k_{2}=2.6 \times 10^{-5}$. It is important to note that transient performance has been optimized subject to a stability constraint.

\section{Simulation results}

Assuming no modeling error and initializing the closedloop system off of the periodic orbit-with the initial velocity $10 \%$ higher than its value on the periodic orbit-yields the simulation data presented in Figs. 23 through 25. The landing configuration is being modified at each stride. The orientation of the support hip and the torso vary slightly stride-to-stride under the event-based feedback. The deviation in the flight phase trajectory-compare Figs. 23 and 24 to Figs. 10 and 11 - is clearly much less under the event-based control action. Consequently, the torques during the flight phase are noticeably reduced; see Fig. 25.

The evolution of $\zeta$ from stride-to-stride over the course of the simulation is presented in Fig. 26. The desired convergence rate has been achieved.

The evolution of the event-based-control action, $w_{1}+w_{2}$, is presented in Fig. 27. The induced variation in the landing configuration is rather small. Despite this, there are significant improvements in the rate of convergence to the periodic orbit and the reduction in peak torque. This sensitivity points to potential problems when implementation is pursued on the actual mechanism. It may be necessary to develop other methods to avoid unnecessarily high torques in the flight phase.

\section{Conclusions}

A time-invariant feedback control strategy has been developed for a bipedal runner. The control strategy is hybrid: it
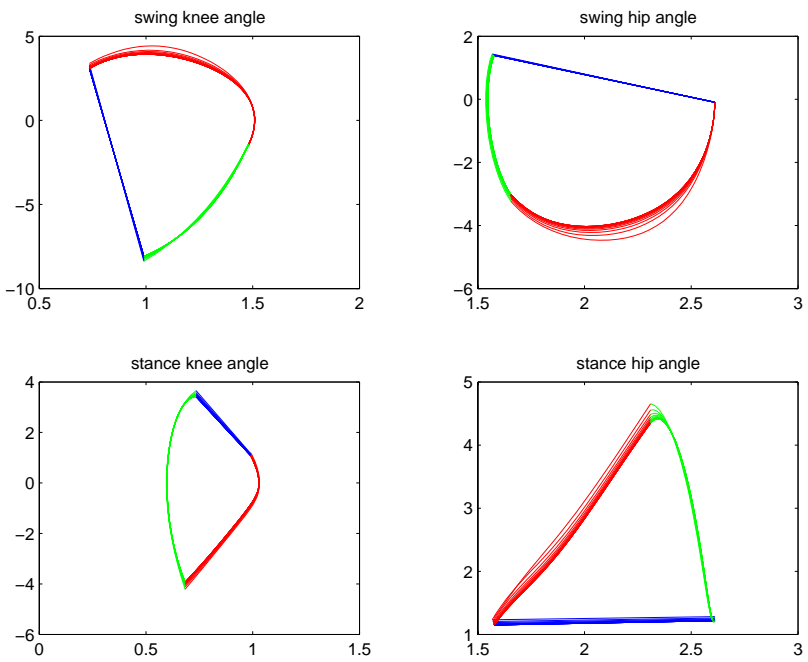

Fig. 23. Running at $1.5 \mathrm{~m} / \mathrm{s}$ with event-based control of the landing configuration. The four graphs depict the relative joint angles in radians ( $\mathrm{x}-$ axis) versus their velocities in radians per second (y-axis) in the stance, flight and impact phases: the swing knee angle (knee of leg-2), the swing hip angle (hip of leg-2), the stance knee angle (knee of leg-1) and the stance hip angle (hip of leg-1). At impact, the roles of the limbs are exchanged; as a consequence the configuration angles change at impact; see (30). Notice that the robot no longer has the same configuration at each transition between phases. The plots indicate that a limit cycle is achieved.

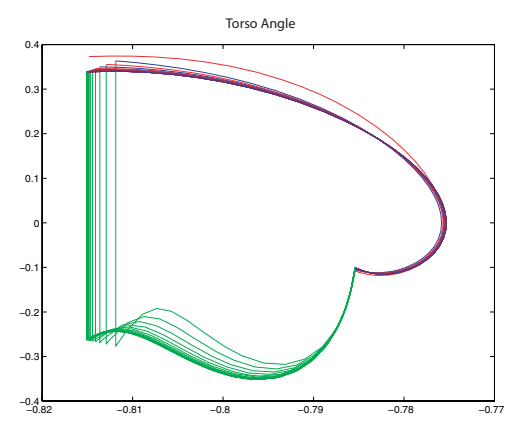

Fig. 24. Running at $1.5 \mathrm{~m} / \mathrm{s}$ with event-based control of the landing configuration. The graph depicts torso angle in radians (x-axis) versus its velocity in radians per second (y-axis) in the stance and flight phases. Notice that the torso angle at the end of the flight phase varies stride-to-stride. The plot indicates that a limit cycle is achieved.

acts both continuously during a stride and discretely stride-tostride. The flight-phase portion of the control strategy was designed so as to create a generalized impact map whose properties are similar to those of the impact maps that occur in models of walking. This led to the deliberate design of a hybrid zero dynamics of running, that is, a low-dimensional, invariant, sub-dynamic of the closed-loop hybrid system. Asymptotically stable orbits of the hybrid zero dynamics are asymptotically stabilizable orbits of the full-order hybrid model. Using the idea of a restricted Poincaré return map-which is the Poincaré return map associated with the hybrid zero dynamics-an explicit criterion for the existence of periodic orbits was given, as well as an explicit characterization of their stability properties. With this theoretical tool, stability analysis involves the straightforward computation of a scalar map.

The principal results were illustrated on a five-link, fouractuator planar biped with revolute joints. Periodic trajectories 

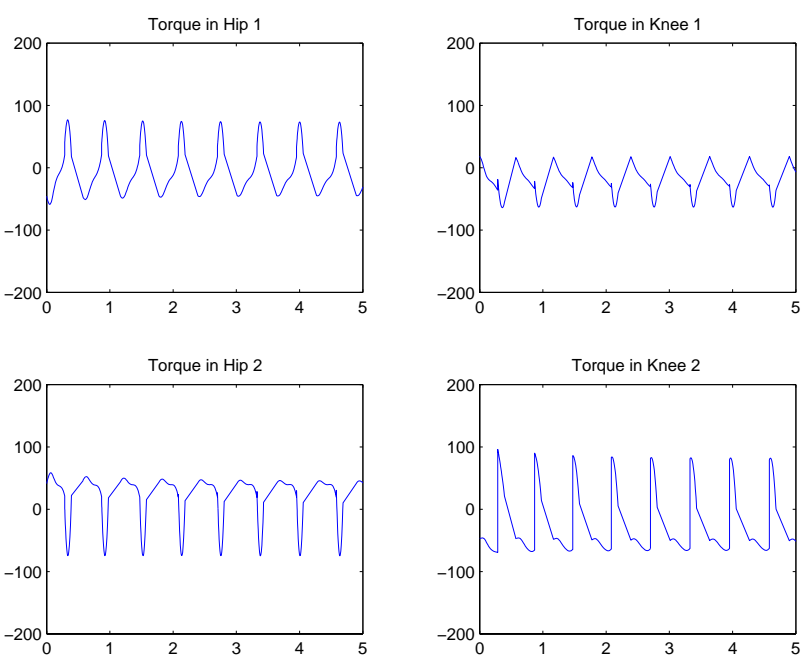

Fig. 25. Running at $1.5 \mathrm{~m} / \mathrm{s}$ with event-based control of the landing configuration. The four graphs depict the joint torques in Newton-meters (yaxis) versus time in seconds (x-axis) in the stance and flight phases. Modifying the landing configuration stride-to-stride has resulted in much smaller torques when the robot is off of the periodic orbit.

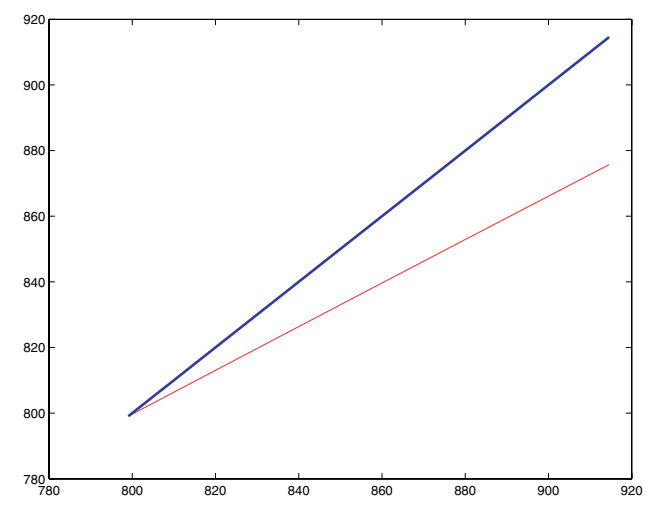

Fig. 26. Running at $1.5 \mathrm{~m} / \mathrm{s}$ with event-based control of the landing configuration. The graph's thick line depicts the value of $\zeta$ at step $k+1$ (y-axis) versus its value at step $k$ (x-axis) as obtained directly from the simulation. The desired modification in the slope of the Poincaré map has been obtained without changing the fixed point: slope $\approx 0.66, \zeta^{*} \approx 800$. The thin line is the identity map. The fixed point is at the intersection of the two lines.

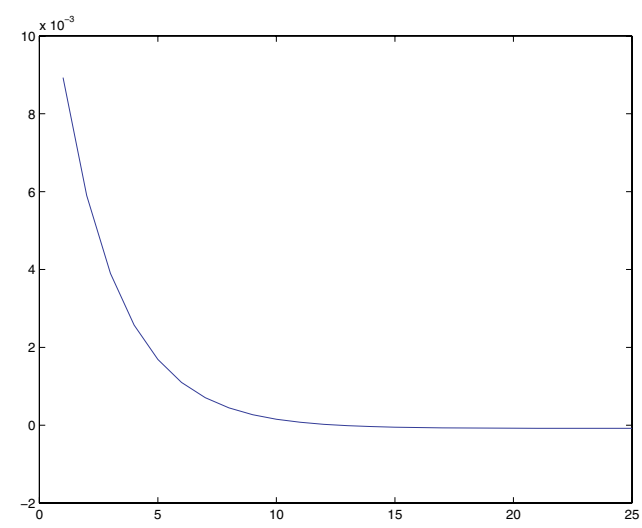

Fig. 27. Running at $1.5 \mathrm{~m} / \mathrm{s}$ with event-based control of the landing configuration. The graph depicts $w_{1}$ (y-axis) versus step number (x-axis) as obtained in the simulation. Note that $w_{2}=\frac{k_{2}}{k_{1}} w_{1}$. computed in [11] were interpreted as desired periodic orbits in the state space of the robot model. For each trajectory, a time-invariant feedback controller was designed to realize the corresponding orbit as an attractive solution of the hybrid zero dynamics, without, it is very important to note, the use of trajectory tracking. Very roughly speaking, the controller is "clocked" to events on the orbit and not to time. Hence, when perturbed away from the orbit, the robot's links regain "synchrony" with respect to the robot's position on the orbit and not with respect to time. In this sense, the work here is philosophically similar to [15]-[18], [55] and diametrically opposed to most other work in the legged-locomotion literature.

The performance of the controller was simulated on slow and fast running motions. Robustness of the controller to model imperfections was demonstrated through simulations where the rigid impact model was replaced by a compliant contact model, and where the parameters of the robot model did not correspond to those used to design the controller. The event-based control method of [4], [13] was used to accelerate the rate of convergence to a periodic orbit and to improve the transient performance in the flight phase.

This work has only partially explored the benefits of approaching the feedback control of running with tools previously developed for walking. On the basis of [4], it seems likely that event-based control can be profitably used in running to provide additional regulation of average running speed and rejection of perturbations. These extensions may involve event-based control actions in the stance-phase controller, parallel to what was done here for the flight-phase controller.

While this paper was in review, experimental implementation of a running controller was initiated [56].

\section{ACKNOWLEDGMENTS}

The work of E.R. Westervelt and J.W. Grizzle was supported by NSF grants CMS-0408348 and ECS-0322395, respectively.

\section{REFERENCES}

[1] C. Chevallereau, G. Abba, Y. Aoustin, E. R. Plestan, F. Westervelt, C. Canduas-de Wit, and J. W. Grizzle, "RABBIT: A testbed for advanced control theory," IEEE Control Systems Magazine, vol. 23, no. 5, pp. 5779, October 2003

[2] J. W. Grizzle, G. Abba, and F. Plestan, "Asymptotically stable walking for biped robots: Analysis via systems with impulse effects," IEEE Transactions on Automatic Control, vol. 46, pp. 51-64, January 2001.

[3] E. R. Westervelt, J. W. Grizzle, and D. E. Koditschek, "Hybrid zero dynamics of planar biped walkers," IEEE Transactions on Automatic Control, vol. 48, no. 1, pp. 42-56, January 2003.

[4] E. R. Westervelt, J. W. Grizzle, and C. Canudas-de-Wit, "Switching and PI control of walking motions of planar biped walkers," IEEE Transactions on Automatic Control, vol. 48, no. 2, pp. 308-312, February 2003.

[5] F. Plestan, J. W. Grizzle, E. R. Westervelt, and G. Abba, "Stable walking of a 7-DOF biped robot," IEEE Transactions on Robotics and Automation, vol. 19, no. 4, pp. 653-668, August 2003.

[6] C. Chevallereau, "Time scaling control for an underactuated biped robot," IEEE Transactions on Robotics and Automation, vol. 18, April 2003.

[7] C. Chevallereau, A. Formal'sky, and D. Djoudi, "Tracking of a joint path for the walking of an underactuated biped," Robotica, vol. 22, pp. 15-28, 2004.

[8] E. R. Westervelt, G. Buche, and J. W. Grizzle, "Experimental validation of a framework for the design of controllers that induce stable walking in planar bipeds," International Journal of Robotics Research, June 2004. 
[9] — , "Inducing dynamically stable walking in an underactuated prototype planar biped," in Proc. of the IEEE International Conference on Robotics and Automation, New Orleans, LA, May 2004, see [?] for a reprint.

[10] C. Chevallereau and P. Sardain, "Design and actuation optimization of a 4 axes biped robot for walking and running," in Proc. of the IEEE International Conference on Robotics and Automation, San Francisco, California, April 2000, pp. 3365-3370.

[11] C. Chevallereau and Y. Aoustin, "Optimal reference trajectories for walking and running of a biped robot," Robotica, vol. 19, no. 5, pp. 557-569, September 2001.

[12] E. R. Westervelt, "Toward a coherent framework for the control of planar biped locomotion," Ph.D. dissertation, University of Michigan, Ann Arbor, June 2003.

[13] J. W. Grizzle, E. R. Westervelt, and C. Canudas-de-Wit, "Event-based pi control of an underactuated biped walker," in IEEE Conf. on Decision and Control. IEEE Press, December 2003.

[14] Supplemental material for this article, "available at [?]," March 2004.

[15] M. Raibert, Legged robots that balance. Mass.: MIT Press, 1986.

[16] — "Legged robots," Communications of the ACM, vol. 29, no. 6, pp. 499-514, 1986

[17] D. E. Koditschek and M. Buehler, "Analysis of a simplified hopping robot," International Journal of Robotics Research, vol. 10, no. 6, pp. 587-605, 1991 .

[18] C. Francois and C. Samson, "A new approach to the control of the planar one-legged hopper," International Journal of Robotics Research, vol. 17, no. 11 , pp. 1150-1166, 1998.

[19] M. Ahmadi and M. Buehler, "Stable control of a simulated one-legged running robot with hip and leg compliance," IEEE Transactions on Robotics and Automation, vol. 13, no. 1, pp. 96-104, February 1997.

[20] J. Hodgins and M. Raibert, "Adjusting step length for rough terrain locomotion," IEEE Transactions on Robotics and Automation, vol. 7(3), pp. 289-298, June 1991.

[21] J. Nakanishi, T. Fukuda, and D. E. Koditschek, "A brachiating robot controller," IEEE Transactions on Robotics and Automation, vol. 16, no. 2, pp. 109-123, April 2000.

[22] M. Buehler, D. E. Koditschek, and P. Kindlmann, "A family of robot control strategies for intermittent dynamical environments," IEEE Control Systems Magazine, vol. 10, no. 2, pp. 16-22, Feb 1990.

[23] A. Rizzi and D. E. Koditschek, "An active visual estimator for dexterous manipulation," IEEE Transactions on Robotics and Automation, vol. 12, no. 5, pp. 697-713, October 1996.

[24] R. Full and D. E. Koditschek, "Templates and anchors: Neuromechanical hypotheses of legged locomotion on land," Journal of Experimental Biology, vol. 202, pp. 3325-3332, December 1999.

[25] U. Saranli, W. Schwind, and D. E. Koditschek, "Toward the control of a multi-jointed, monopod runner,' in Proc. of the IEEE International Conference on Robotics and Automation, Leuven, Belgium, May 1998, pp. 2676-2682.

[26] U. Saranli, "Dynamic locomotion with a hexapod robot," Ph.D. dissertation, University of Michigan, 2002.

[27] I. Kolmanovsky, N. McClamroch, and V. Coppola, "New results on control of multibody systems which conserve angular momentum," Journal of Dynamical and Control Systems, vol. 1(4), pp. 447-462, 1995.

[28] J. Godhavn, A. Balluchi, L. Crawford, and S. Sastry, "Path planning for nonholonmic sysetms with drift," in Proc. of the American Control Conference, Albuguerque, NM, June 1997, pp. 532-536.

[29] M. Sampei, H. Kiyota, and M. Ishikawa, "Control strategies for mechanical systems with various constraints-control of nonholonomic systems," in IEEE Conf. on Systems, Man, and Cybernetics, III, 1999, pp. 158167.

[30] M. Miyazaki, M. Sampei, and M. Koga, "Control of a motion of an acrobot approaching a horizontal bar," Advanced Robotics, vol. 15(4), pp. 467-480, 2001.

[31] C. Chevallereau, J. W. Grizzle, and C. H. Moog, "Nonlinear control of mechanical systems with one degree of underactuation," in Proc. of the IEEE International Conference on Robotics and Automation, New Orleans, LA, April 2004.

[32] S. Kajita, T. Nagasaki, and K. Kaneko, "A hop towards running humanoid robot," in Proc. of the IEEE International Conference on Robotics and Automation, New Orleans, LA, April 2004.

[33] "Johnnie - The TUM Biped Walking Robot," March 2005, www.amm. mw.tu-muenchen.de/Forschung/ZWEIBEINER/johnnie_e.html.

[34] F. Pfeiffer, K. Loffler, and M. Gienger, "The concept of jogging johnnie," in Proc. of the IEEE International Conference on Robotics and Automation, Washington, DC, May 2002.
[35] ROBEA: Robotics and Artificial Entities, "robot-rabbit.lag.ensieg.inpg. fr/index.php (in French; for an English version, click on the British flag)," February 2004

[36] T. Fujimoto, "Trajectory generation of biped running robot with minimum energy consumption," in Proc. of the IEEE International Conference on Robotics and Automation, New Orleans, LA, April 2004, pp. 3803-3808.

[37] S. Kajita and T. Nagasaki, "Running pattern generation for a humanoid robot," in Proc. of the IEEE International Conference on Robotics and Automation, Washington, DC, May 2002, pp. 2755-2761.

[38] S. Hyon and T. Emura, "Running control of a planar biped robot based on energy-preserving strategy," in Proc. of the IEEE International Conference on Robotics and Automation, New Orleans, LA, April 2004, pp. 3791-3796.

[39] H. Goldstein, Classical Mechanics, 2nd ed. Addison Wesley, 1980.

[40] M. Spong and M. Vidyasagar, Robot dynamics and control. New York: John Wiley and Sons, 1991.

[41] E. Dombre and W. Khalil, Modeling, identification and control of robots, ser. Hermes Sciences, Europe, Paris, 2002.

[42] A. Formal'sky, Locomotion of Anthropomorphic Mechanisms, ser. Nauka. Moscow: in Russian, 1982.

[43] Y. Hurmuzlu and D. Marghitu, "Rigid body collisions of planar kinematic chains with multiple contact points," International Journal of Robotics Research, vol. 13, no. 1, pp. 82-92, 1994.

[44] J. Guckenheimer and S. Johnson, "Planar hybrid systems," in Hybrid Systems II, Lecture Notes in Computer Science. Springer-Verlag, 1995, pp. 203-225.

[45] S. G. Nersesov, V. Chellaboina, and W. M. Haddad, "A generalization of Poincaré's theorem to hybrid and impulsive dynamical systems," Int. J. Hybrid Systems, vol. 2, pp. 35-51, 2002.

[46] A. V. Roup, D. S. Bernstein, S. G. Nersesov, W. M. Haddad, and V. Chellaboina, "Limit cycle analysis of the verge and foliot clock escapement using impulsive differential equations and poincare maps," Int. J. Control, vol. 76, no. 17, pp. 1685-1698, 2003.

[47] A. Isidori, Nonlinear Control Systems: An Introduction, 3rd ed. Berlin: Springer-Verlag, 1995.

[48] B. Espiau and A. Goswami, "Compass gait revisited," in Proc. of the IFAC Symposium on Robot Control, Capri, Italy, September 1994, pp. 839-846.

[49] I. Hiskens, "Stability of hyrbid limit cycles: application to the compass gait biped robot," in Proc. of the 40th IEEE Conf. Dec. and Control, Orlando, FL, December 2001, pp. 774-779.

[50] T. Parker and L. Chua, Practical Numerical Algorithms for Chaotic Systems. New York: Springer-Verlag, 1989.

[51] D. Bainov and P. Simeonov, Systems with Impulse Effects : Stability, Theory and Applications. Chichester: Ellis Horwood Limited, 1989.

[52] H. Ye, A. Michel, and L. Hou, "Stability theory for hybrid dynamical systems," IEEE Transactions on Automatic Control, vol. 43, no. 4, pp. 461-474, April 1998.

[53] D. Singer, "Stable orbits and bifuractions of maps of the interval," SIAM Journal of Applied Mathematics, vol. 35, no. 2, pp. 260-267, 1978.

[54] J. Guckenheimer, "Sensitive dependence to initial conditins for one dimensional maps," Communications in Mathematical Physics, vol. 70, pp. 133-160, 1979.

[55] M. Buehler, D. E. Koditschek, and P. Kindlmann, "Planning and control of a juggling robot," International Journal of Robotics Research, vol. 13, no. 2, pp. 101-118, 1994.

[56] B. Morris, E. R. Westervelt, C. Chevallereau, G. Buche, and J. W. Grizzle, "Achieving bipedal running with RABBIT: Six steps toward infinity," 2005, under review. Paper submitted to Robotics: Science \& Systems (Robotics2005) on January 21, 2005. Available at [?].

[57] S. P. Bhat and D. S. Bernstein, "Continuous finite-time stabilization of the translational and rotational double integrators," IEEE Transactions on Automatic Control, vol. 43, no. 5, pp. 678-682, 1998.

[58] V. Haimo, "Finite time controllers," SIAM J. Control and Optimization, vol. 24 , no. 4, pp. 760-770, 1986.

[59] S. P. Bhat and D. S. Bernstein, "Finite-time stability of continuous autonomous systems," SIAM Journal of Control and Optimization, vol. 38 , no. 3, pp. 51-766, 2000.

\section{APPENDIX I}

\section{Stance and Flight Phase Control Laws}

The control laws are rewritten here in a form where their dependence on terms in the models becomes more explicit. 


\section{A. Stance phase control}

The control law is defined so as to achieve input-output linearization with respect to $v, \frac{d^{2} y_{\mathrm{s}}}{d t^{2}}=v\left(y_{\mathrm{s}}, \dot{y}_{\mathrm{s}}\right)$, where $v\left(y_{\mathrm{s}}, \dot{y}_{\mathrm{s}}\right)$ is subsequently chosen to globally asymptotically stabilize the origin with finite-time convergence [2]. Inputoutput linearization is equivalent to

$$
\ddot{q}_{\mathrm{b}}=\frac{\partial h_{d, \mathrm{~s}}}{\partial \theta_{\mathrm{s}}} \ddot{\theta}_{\mathrm{s}}+\frac{\partial^{2} h_{d, \mathrm{~s}}}{\partial \theta_{\mathrm{s}}^{2}} \dot{\theta}_{\mathrm{s}}^{2}+v \text {. }
$$

The robot's configuration is a linear combination of $q_{\mathrm{b}}$ and $\theta_{\mathrm{s}}$,

$$
q=\mathbf{T}\left[\begin{array}{l}
q_{\mathrm{b}} \\
\theta_{\mathrm{s}}
\end{array}\right],
$$

where $\mathbf{T}$ is a constant invertible matrix, and hence the control law is easily expressed directly in terms of the dynamic model. Indeed, the required torques satisfy

$$
\begin{aligned}
& D_{\mathrm{s}}\left(q_{\mathrm{b}}\right) \mathbf{T}\left[\begin{array}{c}
\frac{\partial h_{d, \mathrm{~s}}}{\partial \theta_{\mathrm{s}}} \ddot{\theta}_{\mathrm{s}}+\frac{\partial^{2} h_{d, \mathrm{~s}}}{\partial \theta_{\mathrm{s}}^{2}} \dot{\theta}_{\mathrm{s}}^{2}+v \\
\ddot{\theta}_{\mathrm{s}}
\end{array}\right] \\
& +C_{\mathrm{s}}\left(q_{\mathrm{b}}, \dot{q}\right) \dot{q}+G_{\mathrm{s}}(q)=\left[\begin{array}{c}
I_{4 \times 4} \\
0
\end{array}\right] u .
\end{aligned}
$$

The fifth row of the model yields $\ddot{\theta}_{\mathrm{s}}$. Substituting this expression in the above yields the required feedback controller

$$
\begin{gathered}
u_{\mathrm{s}}=-D_{\mathrm{s}, 1: 4}\left(q_{\mathrm{b}}\right) \mathbf{T}\left[\begin{array}{c}
\frac{\partial h_{d, \mathrm{~s}}}{\partial \theta_{\mathrm{s}}} \\
1
\end{array}\right]\left(D_{\mathrm{s}, 5}\left(q_{\mathrm{b}}\right) \mathbf{T}\left[\begin{array}{c}
\frac{\partial h_{d, \mathrm{~s}}}{\partial \theta_{\mathrm{s}}} \\
1
\end{array}\right]\right)^{-1} \\
\left(D_{\mathrm{s}, 5}\left(q_{\mathrm{b}}\right) \mathbf{T}\left[\begin{array}{c}
\frac{\partial^{2} h_{d, \mathrm{~s}}}{\partial \theta_{\mathrm{s}}^{2}} \dot{\theta}_{\mathrm{s}}^{2}+v \\
0
\end{array}\right]+C_{\mathrm{s}, 5}\left(q_{\mathrm{b}}, \dot{q}\right) \dot{q}+G_{\mathrm{s}, 5}(q)\right) \\
+D_{\mathrm{s}, 1: 4}\left(q_{\mathrm{b}}\right) \mathbf{T}\left[\begin{array}{c}
\frac{\partial^{2} h_{d, \mathrm{~s}}}{\partial \theta_{\mathrm{s}}^{2}} \dot{\theta}_{\mathrm{s}}^{2}+v \\
0
\end{array}\right] \\
+C_{\mathrm{s}, 1: 4}\left(q_{\mathrm{b}}, \dot{q}\right) \dot{q}+G_{\mathrm{s}, 1: 4}(q),
\end{gathered}
$$

where the subscript ", $1: 4$ " designates the sub-matrix composed of the first four rows and the subscript ", 5 " designates the fifth row only.

\section{B. Flight phase control}

The control law is defined so as to achieve input-output linearization, $\frac{d^{2} y_{\mathrm{f}}}{d t^{2}}=v\left(y_{\mathrm{f}}, \dot{y}_{\mathrm{f}}\right)$, where $v\left(y_{\mathrm{f}}, \dot{y}_{\mathrm{f}}\right)=-K_{p} y_{\mathrm{f}}-$ $K_{d} \dot{y}_{\mathrm{f}}$ exponentially stabilizes the double integrator. Inputoutput linearization is equivalent to

$$
\ddot{q}_{\mathrm{b}}=\frac{\partial^{2} h_{d, \mathrm{f}}\left(\mathbf{x}_{\mathrm{cm}}, a_{\mathrm{f}}\right)}{\partial \mathbf{x}_{\mathrm{cm}}^{2}} \dot{\mathbf{x}}_{\mathrm{cm}}^{2}+v,
$$

because $a_{\mathrm{f}}$ is constant during the flight phase and $\ddot{\mathbf{x}}_{\mathrm{cm}}=0$. The control laws is easily determined using the first five rows of the dynamic model:

$$
\begin{array}{r}
u_{\mathrm{f}}=\left(A_{1: 4,1: 4}-A_{1: 4,5} A_{5,5}^{-1} A_{5,1: 4}\right)\left(\frac{\partial^{2} h_{d, \mathrm{f}}}{\partial \mathbf{x}_{\mathrm{cm}}^{2}} \dot{\mathbf{x}}_{\mathrm{cm}}^{2}+v\right) \\
+C_{\mathrm{f}, 1: 4}-A_{1: 4,5} A_{5,5}^{-1} C_{\mathrm{f}, 5},
\end{array}
$$

where the subscript " $1: 4,1: 4$ " designates the square submatrix composed of the first four rows and columns, the subscript " $1: 4,5$ " denotes the first four rows of the fifth column, the subscript " $5,1: 4$ "denotes the first four columns of the fifth row and the subscript ", 5 " designates the fifth row only.

\section{APPENDIX II}

\section{Hypotheses ON THE Finite-TIME CONTROLLER IN THE STANCE Phase}

Let $v(y, \dot{y})$ be any feedback controller on (36) satisfying conditions $\mathrm{CH} 2-\mathrm{CH} 5$ of [2], that is, for $N=4$ the dimension of $y$ :

Controller Hypotheses: for the closed-loop chain of double integrators, $\ddot{y}=v(y, \dot{y})$,

$\mathrm{CH} 2$ ) solutions globally exist on $\mathbb{R}^{2 N-2}$, and are unique;

$\mathrm{CH} 3$ ) solutions depend continuously on the initial conditions;

CH4) the origin is globally asymptotically stable, and convergence is achieved in finite time;

CH5) the settling time function ${ }^{11}, T_{\text {set }}: \mathbb{R}^{2 N-2} \rightarrow \mathbb{R}$ by

$$
\begin{array}{r}
T_{\text {set }}\left(y_{0}, \dot{y}_{0}\right):=\inf \{t>0 \mid(y(t), \dot{y}(t))=(0,0), \\
\left.(y(0), \dot{y}(0))=\left(y_{0}, \dot{y}_{0}\right)\right\}
\end{array}
$$

depends continuously on the initial condition, $\left(y_{0}, \dot{y}_{0}\right)$.

Hypotheses $\mathrm{CH} 2-\mathrm{CH} 4$ correspond to the definition of finitetime stability [57], [58]; $\mathrm{CH} 5$ is also needed, but is not implied by $\mathrm{CH} 2-\mathrm{CH} 4$ [59]. These requirements rule out traditional sliding mode control, with its well-known discontinuous action.

\section{APPENDIX III}

\section{PROOF OF THEOREM 2}

By (41), points in $\tilde{\mathcal{S}} \cap \mathcal{Z}_{\mathrm{s}}$ are parameterized by $\left(q_{0}^{\mathrm{s}-}, \dot{q}_{0}^{\mathrm{s}-} \sigma_{1}^{\mathrm{s}-}\right)$. The position of the center of mass $\left(\mathbf{x}_{\mathrm{cm}}^{\mathrm{s}-}, \mathbf{y}_{\mathrm{cm}}^{\mathrm{s}-}\right)$ is obtained by evaluating (8) at $q_{0}^{\mathrm{s}-}$ and its velocity is obtained from (46), $\left(\dot{\mathbf{x}}_{\mathrm{cm}}^{\mathrm{s}-}, \dot{\mathbf{y}}_{\mathrm{cm}}^{\mathrm{s}-}\right)=\left(\lambda_{x}\left(q_{0}^{\mathrm{s}-}\right) \sigma_{1}^{\mathrm{s}-}, \lambda_{y}\left(q_{0}^{\mathrm{s}-}\right) \sigma_{1}^{\mathrm{s}-}\right)$. The angular momentum about the center of mass can be determined from (24) to be

$$
\sigma_{\mathrm{cm}}^{\mathrm{s}-}=\sigma_{1}^{\mathrm{s}-}-m\left(\mathbf{y}_{\mathrm{cm}}^{\mathrm{s}-} \lambda_{x}\left(q_{0}^{\mathrm{s}-}\right) \sigma_{1}^{\mathrm{s}-}-\mathbf{x}_{\mathrm{cm}}^{\mathrm{s}-} \lambda_{y}\left(q_{0}^{\mathrm{s}-}\right) \sigma_{1}^{\mathrm{s}-}\right) .
$$

Since the transition map from the stance phase to the flight phase preserves positions and velocities, (99) is also the angular momentum at the beginning of the flight phase, $\sigma_{\mathrm{cm}}^{\mathrm{f}+}$, and because angular momentum is conserved during ballistic motion, (99) is also the value of the angular momentum at the end of the flight phase, $\sigma_{\mathrm{cm}}^{\mathrm{f}-}$. From the hypotheses $\Delta\left(\tilde{\mathcal{S}} \cap \mathcal{Z}_{\mathrm{s}}\right) \subset \mathcal{Z}_{\mathrm{s}}$ and $\pi \circ \Delta\left(\tilde{\mathcal{S}} \cap \mathcal{Z}_{\mathrm{s}}\right)$ is a single point, the position of the center of mass at the end of the flight phase is known and equal to the position of the center of mass at the beginning of the subsequent stance phase, $\left(\mathbf{x}_{\mathrm{cm}}^{\mathrm{s}+}, \mathbf{y}_{\mathrm{cm}}^{\mathrm{s}+}\right)$. From this, the flight time, $t_{f}$, can be computed

$$
t_{f}=\frac{\dot{\mathbf{y}}_{\mathrm{cm}}^{\mathrm{s}-}}{g}+\frac{\sqrt{\left(\dot{\mathbf{y}}_{\mathrm{cm}}^{\mathrm{s}-}\right)^{2}-2 g\left(\mathbf{y}_{\mathrm{cm}}^{\mathrm{s}+}-\mathbf{y}_{\mathrm{cm}}^{\mathrm{s}-}\right)}}{g},
$$

\footnotetext{
${ }^{11}$ That is, the time it takes for a solution initialized at $\left(y_{0}, \dot{y}_{0}\right)$ to converge to the origin. The terminology is taken from [57].
} 
and from (23), the velocity of the center of mass at the end of the flight phase is determined

$$
\left[\begin{array}{c}
\dot{\mathbf{x}}_{\mathrm{cm}}\left(t_{f}\right) \\
\dot{\mathbf{y}}_{\mathrm{cm}}\left(t_{f}\right)
\end{array}\right]=\left[\begin{array}{c}
\dot{\mathbf{x}}_{\mathrm{cm}}^{\mathrm{s}-} \\
-\sqrt{\left(\dot{\mathbf{y}}_{\mathrm{cm}}^{\mathrm{s}-}\right)^{2}-2 g\left(\mathbf{y}_{\mathrm{cm}}^{\mathrm{s}+}-\mathbf{y}_{\mathrm{cm}}^{\mathrm{s}-}\right)}
\end{array}\right] .
$$

Equations (99), (101), and (24) allow the angular momentum about the contact point at the end of the flight phase, $\sigma_{2}^{\mathrm{f}-}$, to be evaluated, and then (28) allows the evaluation of the angular momentum about the stance leg at the beginning of the subsequence stance phase. This yields

$$
\begin{gathered}
\sigma_{1}^{\mathrm{s}+}=\sigma_{1}^{\mathrm{s}-}-m\left(\mathbf{y}_{\mathrm{cm}}^{\mathrm{s}-} \lambda_{x}\left(q_{0}^{\mathrm{s}-}\right) \sigma_{1}^{\mathrm{s}-}-\mathbf{x}_{\mathrm{cm}}^{\mathrm{s}-} \lambda_{y}\left(q_{0}^{\mathrm{s}-}\right) \sigma_{1}^{\mathrm{s}-}\right)+ \\
m\left(\mathbf{y}_{\mathrm{cm}}^{\mathrm{s}+} \lambda_{x}\left(q_{0}^{\mathrm{s}-}\right) \sigma_{1}^{\mathrm{s}-}+\right. \\
\left.\mathbf{x}_{\mathrm{cm}}^{\mathrm{s}+} \sqrt{\left(\lambda_{y}\left(q_{0}^{\mathrm{s}-}\right) \sigma_{1}^{\mathrm{s}-}\right)^{2}-2 g\left(\mathbf{y}_{\mathrm{cm}}^{\mathrm{s}+}-\mathbf{y}_{\mathrm{cm}}^{\mathrm{s}-}\right)}\right)
\end{gathered}
$$

which, after simplification, completes the proof.

\section{APPENDIX IV}

\section{PROOF OF THEOREM 3}

¿From [3, Sec. IV], in the coordinates $\left(\theta_{\mathrm{s}}, K_{\mathrm{s}, \text { zero }}=\right.$ $\left.\frac{1}{2}\left(\sigma_{1}\right)^{2}\right)$ for $\mathcal{Z}_{\mathrm{s}}$, the stance-phase zero dynamics can be integrated as

$$
K_{\mathrm{s}, \text { zero }}\left(\theta_{\mathrm{s}}\right)=K_{\mathrm{s}, \text { zero }}\left(\theta_{\mathrm{s}}^{+}\right)-V_{\mathrm{s}, \text { zero }}\left(\theta_{\mathrm{s}}\right) .
$$

Evaluating the above at $\theta_{\mathrm{s}}^{-}$and applying (67) yields the restricted Poincaré map

$$
\rho(\zeta)=\delta_{e}(\zeta)-V_{\mathrm{s}, \text { zero }}\left(\theta_{\mathrm{s}}^{-}\right),
$$

where $\zeta=\frac{1}{2}\left(\sigma_{1}^{\mathrm{s}-}\right)^{2}$. The domain of $\rho$ follows from [3, Thm. 3].

Remark 7: The integration of the stance phase zero dynamics can also be expressed as

$$
K_{\mathrm{s}, \text { zero }}\left(\theta_{\mathrm{s}}\right)+V_{\mathrm{s}, \text { zero }}\left(\theta_{\mathrm{s}}\right)=K_{\mathrm{s}, \text { zero }}\left(\theta_{\mathrm{s}}^{+}\right),
$$

for $\theta_{\mathrm{s}}^{+} \leq \theta_{\mathrm{s}}<\theta_{\mathrm{s}}^{-}$, which is conservation of total "pseudoenergy" during the stance phase; see also Fig. 5.

\section{APPENDIX V \\ PROOF OF THEOREM 4}

By (d) of Corollary 2, the hypotheses imply that $\rho$ is strictly convex, and by (c), $\frac{d \rho}{d \zeta} \leq(\chi-|\beta|)^{2}<1$. Hence, the graph of $\rho$ can have at most one intersection with the graph of the identity function, which implies that there can exist at most one fixed point. Since $\delta_{e}$ and $\rho$ differ by a constant, their derivatives are equal and Corollary 2 applies equally to $\delta_{e}$. Therefore, $\delta_{e}$ is strictly increasing on $\tilde{\mathcal{D}}_{\rho}$, and therefore, if $\tilde{\zeta} \in \tilde{\mathcal{D}}_{\rho}$, then $\zeta \in \tilde{\mathcal{D}}_{\rho}$ for all $\zeta>\tilde{\zeta}$. It follows that $\tilde{\mathcal{D}}_{\rho}$ is unbounded and connected. By Corollary $1, \zeta^{*}$ is exponentially stable.

Let $\zeta \in \tilde{\mathcal{D}}_{\rho}$ be such that $\zeta<\zeta^{*}$. Then, since $\rho$ is strictly increasing on $\tilde{\mathcal{D}}_{\rho}, \rho(\zeta)<\rho\left(\zeta^{*}\right)=\zeta^{*}$. Hence, $\rho^{(k)}(\zeta)$ is a strictly increasing sequence bounded from above, and therefore has a limit. By continuity of $\rho$, this limit is a fixed point of $\rho$, and by uniqueness of the fixed point, $\lim _{k \rightarrow \infty} \rho^{(k)}(\zeta)=\zeta^{*}$. Similarly, let $\zeta \in \tilde{\mathcal{D}}_{\rho}$ be such that $\zeta>\zeta^{*}$. Then $\zeta^{*}=\rho\left(\zeta^{*}\right)<\rho(\zeta)$, and similar reasoning shows that $\lim _{k \rightarrow \infty} \rho^{(k)}(\zeta)=\zeta^{*}$, with the convergence being monotonic.

\section{APPENDIX VI}

\section{EFFECTS OF MEASURING ANGLES IN COUNTER-CLOCKWISE DIRECTION}

If angles are positive when measured in the counterclockwise direction, the following equations are modified.Equation (24) becomes

$$
\sigma_{i}=\sigma_{\mathrm{cm}}+m\left(\left(\mathbf{x}_{\mathrm{cm}}-\mathbf{x}_{i}\right) \dot{\mathbf{y}}_{\mathrm{cm}}-\left(\mathbf{y}_{\mathrm{cm}}-\mathbf{y}_{i}\right) \dot{\mathbf{x}}_{\mathrm{cm}}\right) .
$$

The fifth row of (18) is

$\sigma_{\mathrm{cm}}^{+}-\sigma_{\mathrm{cm}}^{-}=-m\left[\mathbf{y}_{\mathrm{cm}}-\mathbf{y}_{2} \mid-\mathbf{x}_{\mathrm{cm}}+\mathbf{x}_{2}\right]\left[\begin{array}{l}\dot{\mathbf{x}}_{\mathrm{cm}}^{+}-\dot{\mathbf{x}}_{\mathrm{cm}}^{-} \\ \dot{\mathbf{y}}_{\mathrm{cm}}^{+}-\dot{\mathbf{y}}_{\mathrm{cm}}^{-}\end{array}\right]$,

since $\frac{\partial f_{2}}{\partial q_{5}}=\left[\mathbf{y}_{\mathrm{cm}}(q)-\mathbf{y}_{2}(q) \mid-\mathbf{x}_{\mathrm{cm}}(q)+\mathbf{x}_{2}(q)\right]^{\prime}$, and using (105) results in (27). Consequently, Appendix III is modified as follows. The angular momentum about the center of mass can be determined from (105) to be

$$
\sigma_{\mathrm{cm}}^{\mathrm{s}-}=\sigma_{1}^{\mathrm{s}-}+m\left(\mathbf{y}_{\mathrm{cm}}^{\mathrm{s}-} \lambda_{x}\left(q_{0}^{\mathrm{s}-}\right) \sigma_{1}^{\mathrm{s}-}-\mathbf{x}_{\mathrm{cm}}^{\mathrm{s}-} \lambda_{y}\left(q_{0}^{\mathrm{s}-}\right) \sigma_{1}^{\mathrm{s}-}\right) .
$$

This yields

$$
\begin{gathered}
\sigma_{1}^{\mathrm{s}+}=\sigma_{1}^{\mathrm{s}-}+m\left(\mathbf{y}_{\mathrm{cm}}^{\mathrm{s}-} \lambda_{x}\left(q_{0}^{\mathrm{s}-}\right) \sigma_{1}^{\mathrm{s}-}-\mathbf{x}_{\mathrm{cm}}^{\mathrm{s}-} \lambda_{y}\left(q_{0}^{\mathrm{s}-}\right) \sigma_{1}^{\mathrm{s}-}\right)- \\
m\left(\mathbf{y}_{\mathrm{cm}}^{\mathrm{s}+} \lambda_{x}\left(q_{0}^{\mathrm{s}-}\right) \sigma_{1}^{\mathrm{s}-}+\right. \\
\left.\mathbf{x}_{\mathrm{cm}}^{\mathrm{s}+} \sqrt{\left(\lambda_{y}\left(q_{0}^{\mathrm{s}-}\right) \sigma_{1}^{\mathrm{s}-}\right)^{2}-2 g\left(\mathbf{y}_{\mathrm{cm}}^{\mathrm{s}+}-\mathbf{y}_{\mathrm{cm}}^{\mathrm{s}-}\right)}\right)
\end{gathered}
$$

Thus

$$
\delta\left(\sigma_{1}^{\mathrm{s}-}\right)=\chi \sigma_{1}^{\mathrm{s}-}+\sqrt{\left(\beta \sigma_{1}^{\mathrm{s}-}\right)^{2}+\alpha},
$$

with

$$
\begin{aligned}
& \alpha=-2 m^{2} g\left(\mathbf{x}_{\mathrm{cm}}^{\mathrm{s}+}\right)^{2}\left(\mathbf{y}_{\mathrm{cm}}^{\mathrm{s}+}-\mathbf{y}_{\mathrm{cm}}^{\mathrm{s}-}\right) \\
& \beta=m \mathbf{x}_{\mathrm{cm}}^{\mathrm{s}+} \lambda_{y}\left(q_{0}^{\mathrm{s}-}\right) \\
& \chi=1-m \mathbf{x}_{\mathrm{cm}}^{\mathrm{s}-} \lambda_{y}\left(q_{0}^{\mathrm{s}-}\right)-m\left(\mathbf{y}_{\mathrm{cm}}^{\mathrm{s}+}-\mathbf{y}_{\mathrm{cm}}^{\mathrm{s}-}\right) \lambda_{x}\left(q_{0}^{\mathrm{s}-}\right) .
\end{aligned}
$$

and equations (65) and (66) must be changed accordingly. Since the sign of the angular momentum is different, the signs of $\lambda_{x}$ and $\lambda_{y}$ are changed. The numerical evaluation of $\alpha$ and $\chi$ gives the same value whether the positive direction is defined clockwise or counter-clockwise. The sign of $\beta$ is changed, but it does not play any role. The main difference is the sign before the square root; thus the evolution of the absolute value of the angular momentum given by (65) for positive angular momentum and by (109) for negative angular momentum is the same. The remainder of the analysis is based on the evolution of $\zeta=\frac{1}{2}\left(\sigma_{1}^{\mathrm{s}-}\right)^{2}$, and since the sign of the angular momentum does not affect $\zeta$, the analysis and formulas are valid for any choice of positive direction. 


\section{APPENDIX VII \\ NOMENCLATURE}

The subscript "f" corresponds to the flight phase and the subscript "s" corresponds to the stance phase. The superscripts "+" and "-" define the beginning and end of a phase respectively. When they are applied to a variable that is only defined for a single phase, such as $\theta_{\mathrm{s}}$, then there is no ambiguity, as in $\theta_{\mathrm{s}}^{\mathrm{s}+}$. For a variable such as $\mathbf{x}_{\mathrm{cm}}$, which is used in both flight and stance phases, the notation $\mathbf{x}_{\mathrm{cm}}^{\mathrm{s}+}, \mathbf{x}_{\mathrm{cm}}^{\mathrm{s}-}, \mathbf{x}_{\mathrm{cm}}^{\mathrm{f}+}$, and $\mathrm{x}_{\mathrm{cm}}^{\mathrm{f}-}$ is used. The superscript " $*$ " occasionally is used to denote the value of a variable on a periodic orbit; an exception is $u^{*}$, which is a feedback control rendering a zero dynamics manifold invariant.

\begin{tabular}{l|l|l}
\hline Symbol & Meaning & Defined \\
\hline \hline$a_{\mathrm{f}}$ & $\begin{array}{l}\text { parameter in flight-phase virtual } \\
\text { constraints; is up-dated each step }\end{array}$ & Sec. VI-B \\
\hline$A\left(q_{\mathrm{b}}\right)$ & $\begin{array}{l}\text { part of inertia matrix in flight } \\
\text { phase, } A_{i}: i-t h \text { row of } A, \\
A_{i j}=A(i, j)\end{array}$ & Eq. (3) \\
\hline $\mathcal{A}$ & $\begin{array}{l}\text { space where } a_{\mathrm{f}} \text { takes values } \\
\mathcal{A}:=\mathbb{R}^{p}, p \text { number of parameters }\end{array}$ & Sec. VI-B \\
\hline$\alpha$ & parameter defining the function $\delta$ & Eq. (66) \\
\hline$b_{1}, b_{2}$ & $\begin{array}{l}\text { sensitivities of restricted Poincaré } \\
\text { map w.r.t. variations } w_{1} \text { and } w_{2}\end{array}$ & Sec. X \\
\hline$B_{\mathrm{f}}, B_{\mathrm{s}}$ & transforms $u$ into $\Gamma_{\mathrm{f}}$ or $\Gamma_{\mathrm{s}}$ & Eqs. (5), (13) \\
\hline$\beta$ & parameter defining the function $\delta$ & Eq. (66) \\
\hline$C_{\mathrm{f}}, C_{\mathrm{s}}$ & coriolis and centrifugal terms & Secs. III-B, III-C \\
\hline$\chi$ & parameter defining the function $\delta$ & Eq. (66) \\
\hline$D_{\mathrm{f}}, D_{\mathrm{s}}$ & inertia matrices & Secs. III-B, III-C \\
\hline $\mathcal{D}_{\rho}$ & domain of definition of $\rho$ & Eq. (70) \\
\hline$\tilde{\mathcal{D}}_{\rho}$ & domain where $\rho$ has positive slope & Eq. (76) \\
\hline$\delta$ & $\begin{array}{l}\text { function that defines the change of } \\
\text { angular momentum } \sigma_{1} \text { in the } \\
\text { restricted impact map }\end{array}$ & Eq. (65) \\
\hline$\delta_{e}$ & $\begin{array}{l}\text { same as } \delta, \text { but expressed in terms } \\
\text { of energy instead of momentum }\end{array}$ & Eq. (67) \\
\hline$\Delta$ & generalized impact map & Thm. 1 \\
\hline$\Delta_{\mathrm{s}}^{\mathrm{f}}, \Delta_{\mathrm{f}}^{\mathrm{s}}$ & $\begin{array}{l}\text { impact maps determining new } \\
\text { initial conditions at transitions }\end{array}$ & Sec. IV \\
\hline$\Delta_{\text {zero }}$ & restricted impact map & Eqs. (63), (64) \\
\hline$\tilde{\Delta}$ & change in velocity at impact & Sec. III-D \\
\hline $\begin{array}{l}\mathrm{f} \\
\text { parameters }\end{array}$ & Sec. VI-C include the \\
\hline
\end{tabular}

\begin{tabular}{|c|c|c|}
\hline Symbol & Meaning & Defined \\
\hline$f_{\mathrm{cl}, \mathrm{f}}, f_{\mathrm{cl}, \mathrm{s}}$ & closed-loop system & Secs. VI-A, VI-B \\
\hline $\mathbf{f}_{i}$ & $\begin{array}{l}\text { vector function defining the } \\
\text { Cartesian coordinates of the mass } \\
\text { center with respect to the } \\
\text { Cartesian coordinates of leg } i\end{array}$ & Eqs. (8), (16) \\
\hline$f_{\mathrm{f}}, f_{\mathrm{s}}$ & $\begin{array}{l}\text { drift vector fields in flight and } \\
\text { stance phase models }\end{array}$ & Eqs. (7), (15) \\
\hline$f_{\text {zero }}(z)$ & stance-phase zero dynamics & Sec. VI-A \\
\hline fcn & $\begin{array}{l}\text { conveniently parameterized } \\
\text { function used in the flight-phase } \\
\text { controller, } h_{d, \mathrm{f}}\left(\mathbf{x}_{\mathrm{cm}}, a_{\mathrm{f}}\right)= \\
\text { fcn }\left(a_{1}, \cdots, a_{5}\right)(\tau)\end{array}$ & Sec. VIII-C \\
\hline$F_{n}, F_{t}$ & $\begin{array}{l}\text { normal and tangential components } \\
\text { of the ground reaction force }\end{array}$ & Sec. IX-A \\
\hline $\mathcal{F}_{\mathrm{f}}, \mathcal{F}_{\mathrm{f}}$ & open-loop flow on state manifold & Sec. IV \\
\hline$\overline{\mathcal{F}}_{\mathrm{cl}, \mathrm{f}}, \mathcal{F}_{\mathrm{cl}, \mathrm{s}}$ & closed-loop flow on state manifold & Sec. VI-C \\
\hline$g$ & gravity constant & \\
\hline$g_{\mathrm{f}}, g_{\mathrm{s}}$ & $\begin{array}{l}\text { control vector fields in flight and } \\
\text { stance phase models }\end{array}$ & Eqs. (7), (15) \\
\hline$G_{\mathrm{f}}, G_{\mathrm{s}}$ & gravity vector & Sec. III-B \\
\hline$\Gamma_{\mathrm{f}}, \Gamma_{\mathrm{s}}$ & $\begin{array}{l}\text { vector of generalized forces and } \\
\text { torques }\end{array}$ & Secs. III-B, III-C \\
\hline$h_{\mathrm{f}}, h_{\mathrm{s}}$ & $\begin{array}{l}\text { output functions in flight and } \\
\text { stance phases }\end{array}$ & Secs. VI-A, VI-B \\
\hline$h_{d, \mathrm{f}}, h_{d, \mathrm{~s}}$ & $\begin{array}{l}\text { part output function defining a } \\
\text { virtual constraint }\end{array}$ & Sec. VI-A, VI-B \\
\hline$H_{\mathrm{f}}^{\mathrm{s}}, H_{\mathrm{s}}^{\mathrm{f}}$ & $\begin{array}{l}\text { switching condition from } \\
\text { "subscript" to "superscript" }\end{array}$ & Sec. IV \\
\hline $\mathbf{I}$ & $\begin{array}{l}\text { pseudo inertia, defines the zero } \\
\text { dynamics }\end{array}$ & Eq. (40) \\
\hline$I_{R}$ & $\begin{array}{l}\text { intensity of the ground reaction } \\
\text { impulses at impact }\end{array}$ & Sec. III-D \\
\hline$k_{1}, k_{2}$ & $\begin{array}{l}\text { gains of the stride-to-stride } \\
\text { controller }\end{array}$ & Sec. $X$ \\
\hline$K_{d}, K_{p}$ & $\begin{array}{l}\text { derivative and proportional gains } \\
\text { of the flight-phase controller }\end{array}$ & Sec. VI-B \\
\hline$K_{\mathrm{f}}, K_{\mathrm{s}}$ & total kinetic energy & Secs. III-B, III-C \\
\hline$K_{\mathrm{s}, \mathrm{zero}}$ & $\begin{array}{l}\text { kinetic energy for the zero } \\
\text { dynamics in stance phase }\end{array}$ & Eq. (44) \\
\hline$\kappa_{1, \mathrm{f}}$ & $\begin{array}{l}\text { part of zero dynamics in flight } \\
\text { phase }\end{array}$ & Eqs. (49), (50) \\
\hline leg-1 & supporting leg in stance & Fig. 1 \\
\hline leg-2 & $\begin{array}{l}\text { swing leg in stance, leg that } \\
\text { impacts at end of flight }\end{array}$ & Fig. 1 \\
\hline $\mathcal{L}_{\mathrm{f}}, \mathcal{L}_{\mathrm{s}}$ & Lagrangian & Secs. III-B, III-C \\
\hline $\mathcal{L}_{\mathrm{s}, \text { zero }}$ & $\begin{array}{l}\text { Lagrangian for the stance phase } \\
\text { zero dynamics }\end{array}$ & Sec. VI-A \\
\hline$\lambda_{x}, \lambda_{y}$ & $\begin{array}{l}\text { normalized velocity of the center } \\
\text { of mass }\end{array}$ & Eqs. (45), (46) \\
\hline$m$ & total mass of the biped & Sec. III-B \\
\hline$\mu$ & $\begin{array}{l}\text { slope of the restricted Poincaré } \\
\text { return map at its fixed point }\end{array}$ & Eq. (75) \\
\hline$\mu_{d}$ & desired value of $\mu$ & Sec. $X$ \\
\hline$I N$ & natural numbers & Sec. VI-B \\
\hline $\mathcal{O}$ & $\begin{array}{l}\text { periodic orbit viewed as a subset } \\
\text { of the state space }\end{array}$ & Sec. VIII \\
\hline$P$ & Poincaré map & Eq. (61) \\
\hline$P_{\mathrm{f}}, P_{\mathrm{s}}$ & $\begin{array}{l}\text { generalized Poincaré map for a } \\
\text { single phase }\end{array}$ & Sec. VII-A \\
\hline
\end{tabular}




\begin{tabular}{|c|c|c|}
\hline Symbol & Meaning & Defined \\
\hline$\pi$ & $\begin{array}{l}\text { canonical projection that picks off } \\
\text { the configuration variable }\end{array}$ & Sec. VII-B \\
\hline $\begin{array}{l}\varphi_{\mathrm{cl}, \mathrm{f}}\left(t, x_{0}\right) \\
\varphi_{\mathrm{cl}, \mathrm{s}}\left(t, x_{0}\right)\end{array}$ & $\begin{array}{l}\text { integral curve of closed-loop dy- } \\
\text { namics with initial state } x_{0}\end{array}$ & Sec. VII-A \\
\hline$\Phi_{\mathrm{f}}, \Phi_{\mathrm{s}}$ & $\begin{array}{l}\text { coordinate change on configuration } \\
\text { space }\end{array}$ & Secs. VI-A, VI-B \\
\hline$q_{\mathrm{b}}$ & $\begin{array}{l}\text { body coordinates, } \\
q_{\mathrm{b}}=\left(q_{1}, q_{2}, q_{3}, q_{4}\right)^{\prime}\end{array}$ & Fig. 1 \\
\hline$q_{\mathrm{f}}, q$ & $\begin{array}{l}\text { generalized coordinates in the } \\
\text { flight } q_{\mathrm{f}}:=\left(q_{\mathrm{b}}^{\prime}, q_{5}, \mathbf{x}_{\mathrm{cm}}, \mathbf{y}_{\mathrm{cm}}\right)^{\prime} \\
\text { and stance } q:=\left(q_{\mathrm{b}}^{\prime}, q_{5}\right)^{\prime} \text { phases }\end{array}$ & Sec. III-B \\
\hline$q_{0}^{\mathrm{f}-}$ & $\begin{array}{l}\text { reference configuration at the end } \\
\text { of flight; equals } R^{-1} q_{0}^{\mathrm{s}+}\end{array}$ & Eq. (83) \\
\hline$q_{0}^{\mathrm{s}-}$ & $\begin{array}{l}\text { reference configuration at the end } \\
\text { of stance }\end{array}$ & Eq. (41) \\
\hline$q_{0}^{\mathrm{s}+}$ & $\begin{array}{l}\text { reference configuration at the } \\
\text { beginning of stance }\end{array}$ & Secs. VII-A, VIII-A \\
\hline$q_{5}$ & $\begin{array}{l}\text { angle of torso referenced to world } \\
\text { frame, and hence biped's absolute } \\
\text { orientation }\end{array}$ & Fig. 1 \\
\hline$\dot{q}_{0}^{\mathrm{s}-}$ & $\begin{array}{l}\text { normalized reference velocity at } \\
\text { the end of stance }\end{array}$ & Eq. (42) \\
\hline$\dot{q}_{0}^{\mathrm{s}+}$ & $\begin{array}{l}\text { normalized reference velocity at } \\
\text { the beginning of stance }\end{array}$ & Sec. VIII-A \\
\hline$\dot{\bar{q}}_{0}$ & $\begin{array}{l}\text { function determining the velocity } \\
\text { before impact as function of } \\
\text { velocity after impact }\end{array}$ & Eq. (83) \\
\hline $\mathcal{Q}_{\mathrm{f}}, \mathcal{Q}_{\mathrm{s}}$ & configuration space & Secs. III-B, III-C \\
\hline$R$ & $\begin{array}{l}\text { relabeling matrix to take into } \\
\text { account the change of leg number }\end{array}$ & Eq. (31) \\
\hline$\rho$ & restricted Poincaré return map & Eq. (68) \\
\hline $\mathbb{S}$ & topological circle & \\
\hline $\mathcal{S}$ & $\begin{array}{l}\text { switching hyper-surface, also } \\
\text { called an impact hyper-surface, } \\
\mathcal{S}:=\mathcal{S}_{\mathrm{s}}^{\mathrm{f}}\end{array}$ & Thm. 1 \\
\hline $\mathcal{S}_{\mathrm{s}}^{\mathrm{f}}, \mathcal{S}_{\mathrm{f}}^{\mathrm{s}}$ & switching hyper-surface & Sec. IV \\
\hline$\tilde{\mathcal{S}}_{\mathrm{f}}^{\mathrm{s}}, \tilde{\mathcal{S}}_{\mathrm{s}}^{\mathrm{f}}, \tilde{\mathcal{S}}$ & $\begin{array}{l}\text { domains where associated Poincaré } \\
\text { maps are well defined }\end{array}$ & Sec. VII-A, VII-B \\
\hline$\overline{\mathcal{S}}_{\mathrm{f}}^{\mathrm{s}}$ & $\begin{array}{l}\text { switching hyper-surface in the } \\
\text { extended closed-loop model that } \\
\text { includes the parameters as states }\end{array}$ & Sec. VI-C \\
\hline$\sigma_{\mathrm{cm}}$ & $\begin{array}{l}\text { angular momentum of the biped } \\
\text { about its center of mass }\end{array}$ & Sec. III-E \\
\hline$\sigma_{i}$ & $\begin{array}{l}\text { angular momentum of the biped } \\
\text { about the end of leg- } i\end{array}$ & Sec. III-E \\
\hline$\sigma_{1}^{* \mathrm{~s}-}$ & value of $\sigma_{1}^{\mathrm{s}-}$ on a periodic orbit & Sec. VIII-C \\
\hline$\Sigma_{\mathrm{f}}, \Sigma_{\mathrm{s}}$ & $\begin{array}{l}\text { flight and stance portions of } \\
\text { open-loop hybrid model }\end{array}$ & Eq. (29) \\
\hline$\Sigma_{\mathrm{f}, c l}, \Sigma_{\mathrm{s}, c l}$ & $\begin{array}{l}\text { flight and stance portions of } \\
\text { closed-loop hybrid model }\end{array}$ & Eq. (56) \\
\hline$t_{\mathrm{f}}$ & duration of flight & Eq. (100) \\
\hline$T_{I, \mathrm{f}}, T_{I, \mathrm{~s}}$ & $\begin{array}{l}\text { time-to-impact function for one } \\
\text { phase }\end{array}$ & Eq. (57) \\
\hline$T \mathcal{Q}_{\mathrm{f}}, T \mathcal{Q}_{\mathrm{s}}$ & $\begin{array}{l}\text { state space, tangent spaces of } \mathcal{Q}_{\mathrm{f}} \\
\text { and } \mathcal{Q}_{\mathrm{s}}\end{array}$ & Secs. III-B, III-C \\
\hline $\mathbf{T}$ & coordinate transformation matrix & App. I \\
\hline $\mathcal{T}_{\mathrm{s}}^{\mathrm{f}}, \mathcal{T}_{\mathrm{f}}^{\mathrm{s}}$ & $\begin{array}{l}\text { transition function from } \\
\text { "subscript" to "superscript" }\end{array}$ & Sec. IV \\
\hline$\overline{\mathcal{T}}_{\mathrm{f}}^{\mathrm{s}}, \overline{\mathcal{T}}_{\mathrm{s}}^{\mathrm{f}}$ & $\begin{array}{l}\text { transition function from } \\
\text { "subscript" to "superscript" }\end{array}$ & Sec. VI-C \\
\hline
\end{tabular}

\begin{tabular}{|c|c|c|}
\hline Symbol & Meaning & Defined \\
\hline$\tau$ & $\begin{array}{l}\text { scaled time for flight phase, varies } \\
\text { from } 0 \text { to } 1\end{array}$ & Eq. (84) \\
\hline$\theta_{\mathrm{S}}$ & $\begin{array}{l}\text { in stance phase, angle of the hips } \\
\text { with respect the stance leg end }\end{array}$ & Fig. 1 \\
\hline$\theta_{\mathrm{S}}^{-}$ & $\begin{array}{l}\text { value of } \theta_{\mathrm{S}} \text { just before transition to } \\
\text { flight phase }\end{array}$ & Sec. IV \\
\hline$\theta_{\mathrm{s}}^{+}$ & $\begin{array}{l}\text { value of } \theta \text { just after transition } \\
\text { from flight phase }\end{array}$ & Eq. (65) \\
\hline$\theta_{\mathrm{s}, 0}^{-}$ & $\begin{array}{l}\text { desired value of } \theta_{\mathrm{s}} \text { at the transition } \\
\text { from stance to flight; a constant }\end{array}$ & Sec. IV \\
\hline$\theta_{\mathrm{s}, 0}^{+}$ & $\begin{array}{l}\text { desired value of } \theta \text { at the beginning } \\
\text { of stance; a constant }\end{array}$ & Eq. (65) \\
\hline$u$ & vector of actuator torques & Sec. III-B \\
\hline$u_{\mathrm{s}}^{*}$ & $\begin{array}{l}\text { feedback control that renders } \\
\text { stance-phase zero dynamic } \\
\text { manifold invariant }\end{array}$ & Eq. (38) \\
\hline$v$ & $\begin{array}{l}\text { control after partial feedback } \\
\text { linearization }\end{array}$ & Eq. (35) \\
\hline $\mathbf{v}$ & $\begin{array}{l}\text { relative velocity of the end of the } \\
\text { leg with respect to the ground }\end{array}$ & Sec. IX-A \\
\hline$V_{\mathrm{f}}, V_{\mathrm{s}}$ & total potential energy & Secs. III-B, III-C \\
\hline$V_{\mathrm{s}, \text { zero }}$ & $\begin{array}{l}\text { potential energy for the stance } \\
\text { phase zero dynamics }\end{array}$ & Eq. (43) \\
\hline$V_{\mathrm{s}, \mathrm{zero}}^{\max }$ & $\begin{array}{l}\text { maximal value of the potential } \\
\text { energy for the zero dynamics } \\
\text { during stance phase }\end{array}$ & Eq. (71) \\
\hline$w_{\mathrm{s}}^{\mathrm{f}}$ & $\begin{array}{l}\text { transition function for the } \\
\text { flight-phase parameters } a_{\mathrm{f}}\end{array}$ & Sec. VI-C \\
\hline$w_{1}, w_{2}$ & $\begin{array}{l}\text { functions defining the landing } \\
\text { configuration }\end{array}$ & Sec. $X$ \\
\hline$x_{\mathrm{f}}, x_{\mathrm{s}}$ & $\begin{array}{l}\text { state variables for flight and } \\
\text { stance, }\left(q_{\mathrm{f}}^{\prime}, \dot{q}_{\mathrm{f}}^{\prime}\right)^{\prime} \text { and }\left(q^{\prime}, \dot{q}^{\prime}\right)^{\prime}\end{array}$ & Secs. III-B, III-C \\
\hline$x_{\mathrm{f}}^{*-}, x_{\mathrm{s}}^{*-}$ & $\begin{array}{l}\text { state on intersection of periodic } \\
\text { orbit and switching hyper-surface }\end{array}$ & Sec. VIII \\
\hline $\bar{x}_{\mathrm{f}}$ & $\begin{array}{l}\text { extended state in flight phase, } \\
\text { includes the parameters, } \\
\bar{x}_{\mathrm{f}}:=\left(x_{\mathrm{f}}^{\prime}, a_{\mathrm{f}}^{\prime}\right)^{\prime}\end{array}$ & Sec. VI-B \\
\hline $\mathbf{x}_{\mathrm{cm}}$ & $\begin{array}{l}\text { horizontal Cartesian coordinate of } \\
\text { the center of mass }\end{array}$ & Fig. 1 \\
\hline $\mathbf{x}_{1}, \mathbf{x}_{2}$ & $\begin{array}{l}\text { horizontal Cartesian coordinates of } \\
\text { the leg ends }\end{array}$ & Fig. 1 \\
\hline $\mathcal{X}_{\mathrm{f}}, \mathcal{X}_{\mathrm{s}}$ & state space & Sec. IV \\
\hline$\overline{\mathcal{X}}_{\mathrm{f}}$ & $\begin{array}{l}\text { state space including the } \\
\text { parameters }\end{array}$ & Sec. VI-C \\
\hline$y_{\mathrm{f}}, y_{\mathrm{s}}$ & $\begin{array}{l}\text { output used to define the desired } \\
\text { coordination of the limbs }\end{array}$ & Secs. VI-A, VI-B \\
\hline $\mathbf{y}_{\mathrm{cm}}$ & $\begin{array}{l}\text { vertical Cartesian coordinate of the } \\
\text { center of mass }\end{array}$ & Fig. 1 \\
\hline $\mathbf{y}_{1}, \mathbf{y}_{2}$ & $\begin{array}{l}\text { vertical Cartesian coordinates of } \\
\text { leg end }\end{array}$ & Fig. 1 \\
\hline$z$ & $\begin{array}{l}\text { state variable for hybrid zero } \\
\text { dynamics }\end{array}$ & Eq. (39) \\
\hline $\mathbf{z}$ & $\begin{array}{l}\text { penetration depth of leg end in } \\
\text { compliant ground model }\end{array}$ & Eq. (89) \\
\hline $\mathcal{Z}_{\mathrm{f}, a_{\mathrm{f}}}, \mathcal{Z}_{\mathrm{s}}$ & zero dynamics manifold & Secs. VI-A, VI-B \\
\hline$\zeta$ & $\begin{array}{l}\text { kinetic energy for the stance-phase } \\
\text { zero dynamics just before } \\
\text { transition to flight, } \zeta:=\frac{1}{2}\left(\sigma_{1}^{\mathrm{s}-}\right)^{2}\end{array}$ & Sec. VII-B \\
\hline$\zeta^{*}$ & $\begin{array}{l}\text { fixed point of restricted Poincaré } \\
\text { map }\end{array}$ & Eq. (74) \\
\hline
\end{tabular}

